Florida International University

FIU Digital Commons

FIU Electronic Theses and Dissertations

University Graduate School

$11-10-2016$

\title{
Design and Development of Smart Brain-Machine- Brain Interface (SBMIBI) for Deep Brain Stimulation and Other Biomedical Applications
}

Muhammad S. Khan

mkhan055@fiu.edu

DOI: $10.25148 /$ etd.FIDC001261

Follow this and additional works at: https:// digitalcommons.fiu.edu/etd

Part of the Biomedical Commons, Systems and Communications Commons, and the VLSI and Circuits, Embedded and Hardware Systems Commons

\section{Recommended Citation}

Khan, Muhammad S., "Design and Development of Smart Brain-Machine-Brain Interface (SBMIBI) for Deep Brain Stimulation and Other Biomedical Applications" (2016). FIU Electronic Theses and Dissertations. 2724.

https://digitalcommons.fiu.edu/etd/2724 


\section{FLORIDA INTERNATIONAL UNIVERSITY}

Miami, Florida

\section{DESIGN AND DEVELOPMENT OF SMART BRAIN-MACHINE-BRAIN INTERFACE (SBMIBI) FOR DEEP BRAIN STIMULATION AND OTHER BIOMEDICAL APPLICATIONS}

A dissertation submitted in partial fulfillment of the

requirements for the degree of

DOCTOR OF PHILOSOPHY

in

ELECTRICAL ENGINEERING

by

Muhammad S Khan

2016 


\section{To: Interim Dean Ranu Jung}

College of Engineering and Computing

This dissertation, written by Muhammad S Khan, and entitled Design and Development of Smart Brain-Machine-Brain Interface (SBMIBI) for Deep Brain Simulation and Other Biomedical Applications, having been approved in respect to style and intellectual content, is referred to you for judgement.

We have read this dissertation and recommended that it be approved.

\section{Sakhrat Khizroev}

Wei-Chiang Lin

Armando Barreto

Nezih Pala

Ismail Guvenc

Deng Pan

Hai Deng, Major Professor

Date of Defense: November 07, 2016

The dissertation of Muhammad S Khan is approved.

Interim Dean Ranu Jung College of Engineering and Computing

Andrés G. Gil

Vice President for Research and Economic Development and Dean of the University Graduate School

Florida International University, 2016 
CC Copyright 2016 by Muhammad S Khan

All rights reserved. 


\section{DEDICATION}

To my parents, Shahjahan Khan and Anjumanara Khanom! Without their love, affection, dream and inspiration it would not have been possible. 


\section{ACKNOWLEDGMENTS}

I wish to thank my committee members, Drs. Wei-Chiang Lin, Sakhrat Khizroev, Armando Barreto, Nezih Pala, Ismail Guvenc, and Deng Pan for their support and guidance throughout my dissertation work. Especially, I would like to thank my major professor, Dr. Hai Deng for his confidence in my abilities in not only accomplishing the degree work, but

excelling in academic field. Without his continuous support with patience it would be impossible to finish this dissertation work.

Finally, I would also like to thank Mr. Teshaun Francis, Md. Mizanur Rahman, Md. Saiful Islam and Mr. Oscar Silva for their active help throughout my research work. 


\begin{abstract}
OF THE DISSERTATION
DESIGN AND DEVELOPMENT OF SMART BRAIN-MACHINE-BRAIN INTERFACE (SBMIBI) FOR DEEP BRAIN STIMULATION AND OTHER

BIOMEDICAL APPLICATIONS
\end{abstract}

by

Muhammad S Khan

Florida International University, 2016

Miami, Florida

\title{
Professor Hai Deng, Major Professor
}

Machine collaboration with the biological body/brain by sending electrical information back and forth is one of the leading research areas in neuro-engineering during the twentyfirst century. Hence, Brain-Machine-Brain Interface (BMBI) is a powerful tool for achieving such machine-brain/body collaboration. BMBI generally is a smart device (usually invasive) that can record, store, and analyze neural activities, and generate corresponding responses in the form of electrical pulses to stimulate specific brain regions. The Smart Brain-Machine-Brain-Interface (SBMBI) is a step forward with compared to the traditional BMBI by including smart functions, such as in-electrode local computing capabilities, and availability of cloud connectivity in the system to take the advantage of powerful cloud computation in decision making. 
In this dissertation work, we designed and developed an innovative form of Smart BrainMachine-Brain Interface (SBMBI) and studied its feasibility in different biomedical applications. With respect to power management, the SBMBI is a semi-passive platform. The communication module is fully passive - powered by RF harvested energy; whereas, the signal processing core is battery-assisted. The efficiency of the implemented RF energy harvester was measured to be $0.005 \%$.

One of potential applications of SBMBI is to configure a Smart Deep-Brain-Stimulator (SDBS) based on the general SBMBI platform. The SDBS consists of brain-implantable smart electrodes and a wireless-connected external controller. The SDBS electrodes operate as completely autonomous electronic implants that are capable of sensing and recording neural activities in real time, performing local processing, and generating arbitrary waveforms for neuro-stimulation. A bidirectional, secure, fully-passive wireless communication backbone was designed and integrated into this smart electrode to maintain contact between the smart electrodes and the controller. The standard EPC-Global protocol has been modified and adopted as the communication protocol in this design. The proposed SDBS, by using a SBMBI platform, was demonstrated and tested through a hardware prototype. Additionally the SBMBI was employed to develop a low-power wireless ECG data acquisition device. This device captures cardiac pulses through a non-invasive magnetic resonance electrode, processes the signal and sends it to the backend computer through the SBMBI interface. Analysis was performed to verify the integrity of received ECG data. 


\section{TABLE OF CONTENTS}

CHAPTER

PAGE

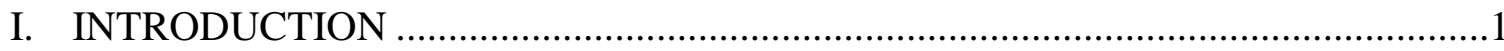

1.1 Brain-Machine-Brain-Interface(BMBI)Overview ................................2

1.2 BMBI in Deep Brain Stimulation (DBS) .......................................... 3

1.3 Challenges in Current DBS systems...............................6 6

1.3.1 Lack of Smartness.................................6

1.3.2 Energy Constraint.................................6

1.3.3 Power Efficient Communication Backbone................ 7

1.3.4 Data Security Enhancement..........................8

1.3.5 Limitation in Stimulatory Waveform Generation...........8

1.3.6 Constraint in True Real-time Neuro Recording..............9

1.4 Next Generation Smart Deep Brain Stimulator (SDBS) ................ 10

1.5 Low Power Wireless ECG Sensing..................................11

1.5.1 Electro Cardiogram (ECG) ..........................11

1.5.2 Wireless Cardiac Activity Monitoring..................12

1.6 Research Objective.............................................. 12

II. RECONFIGURABLE SMART COMMUNICATION PLATFORM.........................13

2.1 Introduction .......................................................................................14

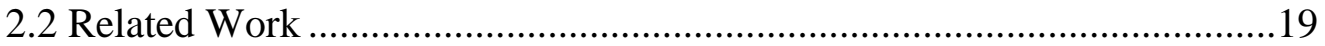

2.3 Design Methodology.................................................22

2.4 System Framework and Implementation..............................24

2.4.1 Tag Platform Configuration .....................................................28

2.4.2 Memory Management ....................................................................31

2.4.3 Modified EPC ID Generation and Sensor Data Communication ..34

2.4.4 Power Budget and Battery Life Time Estimation............................36

2.5 Experimental IoT System .......................................................................

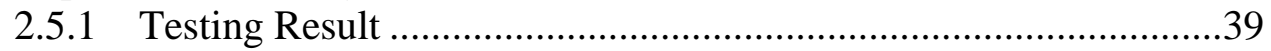

2.6 Conclusions .............................................................................................

III. DESIGN OF AN ENERGY HARVESTING MODULE ……………………..........45

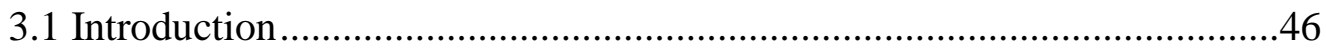

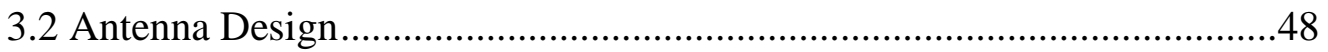

3.3 Energy Harvesting Module Design.......................................................50

3.4 Testing Results .................................................................................5

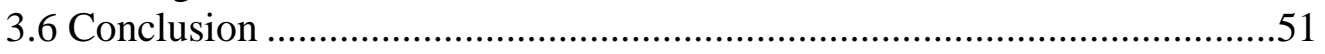

IV. DEVELOPMENT OF SMART DEEP BRAIN STIMULATOR (SDBS) ...................52

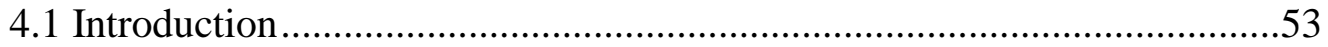

4.2 Smart DBS Architecture …………………………..............................55

4.3 Smart DBS Design and Prototyping....................................59 


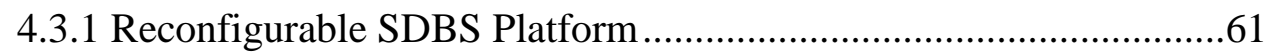

4.3.2 Stimulating Waveform Generation Module.........................................64

4.3.3 Neural Activity Recording \& Processing Module....................68

4.3.4 Data Security Module.......................................... 72

4.3.5 SDBS Powering and Energy Harvesting ..........................73

4.4 SDBS Testing and Evaluation ................................................................74

4.4.1 Waveform Generation........................................ 75

4.4.2 Recording and Processing Module Testing........................76

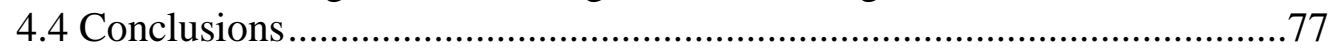

V. DEVELOPMENT OF WIRELESS ECG SENSOR ..............................79

5.1 Introduction...................................................... 80

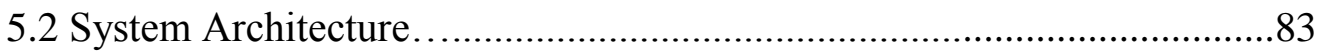

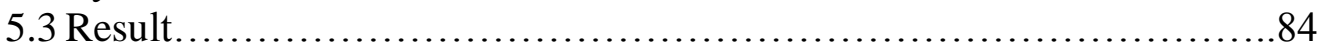

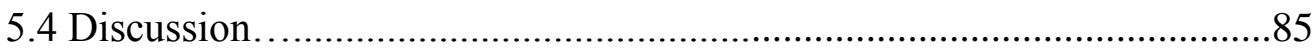

5.5 Conclusions........................................................ 88

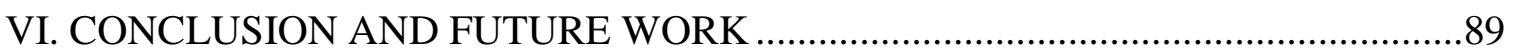

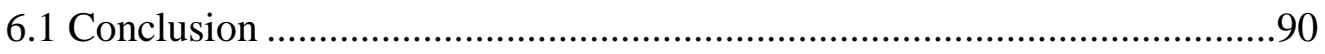

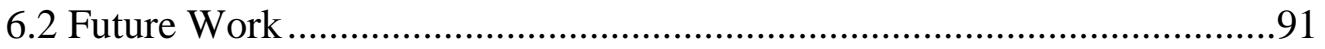

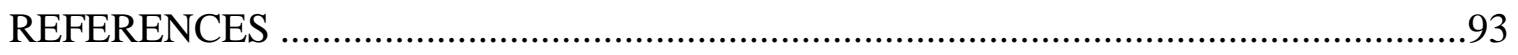

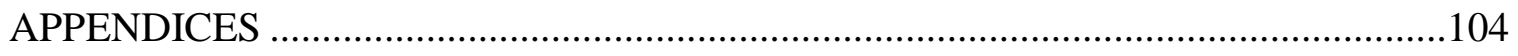

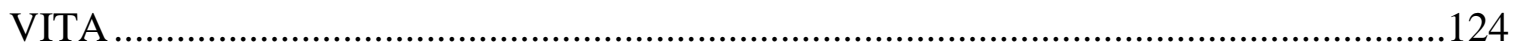




\section{LIST OF TABLES}

TABLE

PAGE

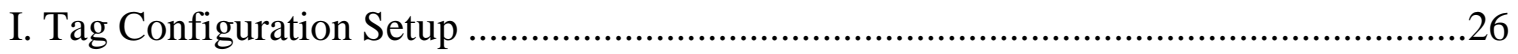

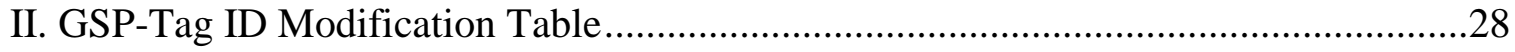

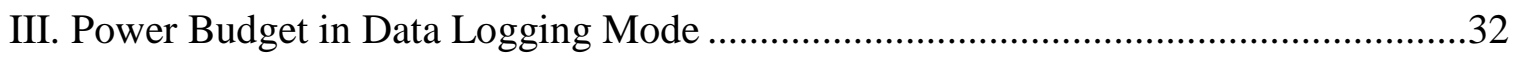

IV. Power Budget in Continuous Data Transmitting Mode .........................................33

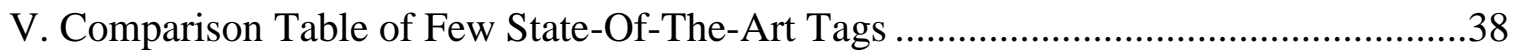

VI. Command Table for the Smart Electrode ............................................................59

VII. Associated Command Table for the Smart Electrode ..........................................60

VIII. Over the ID Communication Layout ..........................................................61

IX. Comparative Study of Few Recent DBS Platforms ..............................................66

X. Instantaneous Samples for Data-Based Arbitrary Waveform Generation ...................67 


\section{LIST OF FIGURES}

FIGURE

PAGE

1. A traditional Brain-Machine-Brain-Interface system. ..........................................

2. Schematic of a Deep Brain Stimulation system ....................................................4

3. A standard ECG signal with "PQRST" complex..................................................11

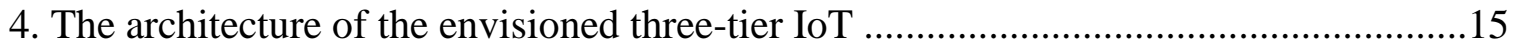

5. (a) Schematic block diagram of the GSP-tag. (b) Second level block diagram of the software defined Digital core, implemented inside a Cortex M3 Microprocessor ............22

6. Implemented GSP-tag on a PCB with the off-the-shelf components ........................25

7. BLF generation performance curve of the encoders ............................................25

8. Schematic diagram of the 1 st configuration block inside the used memory location ...26

9. Firmware state diagram of the GSP's Digital core ...........................................27

10. Memory management algorithm flowchart for the GSP-tag ................................29

11. (a) Schematic of the SSCC-96 EPC ID standard (b) Modified version of the SSCC96 EPC ID Standard. (c) A schematic of the prototyped GSP-tag's transmitted ID .........30

12. The GSP, deployed in a 3-tier IoT system monitoring a package in a smart supply chain monitoring scenario

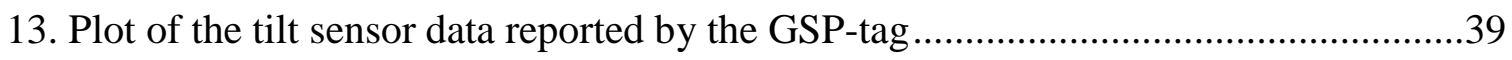

14. Picture shows the snap shot of the Alien reader's software interface showing ...........40

15. Picture shows the snap shot of a conventional G2 read command, reading the User..41

16. Picture shows the snap shot of a conventional G2 read command, reading the User..42

17. Implemented antenna layout and associated radiation pattern and input impedance

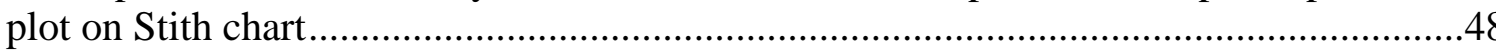


18. (a) Implemented antenna; (b) Simulated and measured return loss; (c) Implemented Cockcroft-Walton charge pump; (d) Snapshot of the test bench to measure harvested power; (e) Harvested power vs distance curve

19. Illustration of the envisioned DBS system.

20. Basic structure of the Smart Deep Brain Stimulation (SDBS) system; (a) Schematic of a smart electrode; (b) Smart electrode's neural activity flow in the data communication layer: monitoring, stimulation generation \& feedback recording; (c) A multi-electrode SDBS system consisting of multiple smart electrode and the external controller where each of the electrodes has been assigned with a unique ID for the controller to target and to communicate with

21. Schematic block diagram of the SDBS System

22. Optional flowchart of the electrode in the smart DBS

23. a) Modified user memory structure of the reconfigurable SDBS platform; b) Memory space management in the conventional Gen2 RFID protocol....

24. Gen2 BlockWrite (data-based waveform generation command) and Write (command-based waveform generation) commands ....

25. (a) Block diagram of the command-based stimulation generation module; (b) schematic diagram of the sigma-delta modulator as D/A converter; and (c) block diagram of the data-based waveform generation module

26. Schematic block diagram of the SDBS recording \& processing module

27. (a) Antenna impedance and radiation pattern; (b) Antenna return loss; (c) Implemented Cockcroft-Walton charge pump; (d) Snapshot of the test bench to measure harvested power; (e) Harvested power vs distance curve.

28. The SDBS system and its components; (a) the smart electro; (b) bottom view of the electrode; (c) SDBS test bench setup where an Alien RFID reader, ALR-9650, is used as the external controller.

29. Maximum communication distance vs detection rate in single \& multi-electrode scenarios. In the multi-electrode case, 5 extra static, electrodes were placed at the approximate $80 \mathrm{~cm}$ away

30. The command shown on the backend server screen is executed for waveform generation using the command-based approach 
31. Smart electrode generated stimulation waveform output; (a) a sinusoidal wave with amplitude $800 \mathrm{mV}$, frequency $2.5 \mathrm{KHz}$ and No. of cycle 5 ; (b) a sinusoidal wave with amplitude $800 \mathrm{mV}$, frequency $2.5 \mathrm{KHz}$ and No. of cycle 10 ; (c) a sinusoidal wave with an amplitude of $800 \mathrm{mV}$, a frequency of $2.5 \mathrm{KHz}$ and the number of cycles equal to20; d) a Gaussian pulse; (e) negative exponential wave; and (f) a positive exponential wave

32. Data-based arbitrary waveform generation; (a) the ideal stimulating waveform to be generated; (b) the schematic structure of the data-based generation command; (c) the actual SBDS-generated waveform

33. SDBS recording and processing module testing results: (a) The input waveform to the SDBS recording module and the waveform regenerated from the recorded data; and (b) SDBS threshold-based detection and stimulation generated from the SDBS closedloop operation (for better representation, input signal wave is shown after the prescalar)

34. Schematic of the proposed ECG monitoring system in real-life deployment situation.

35. Schematic diagram of the ECG signal acquisition circuitry.

36. ECG data acquisition test bench

37. Wireless ECG monitoring interface; (a) Graphical User Interface (GUI) for the wireless ECG monitoring system; (b) Reconstructed ECG signal from the backend computer data-base

38. (a) The "PQRST" complexes in the acquired signal; (b) A standard signal with the "PQRST" complexes . 
CHAPTER I

INTRODUCTION 


\subsection{Brain-Machine-Brain-Interface(BMBI) Overview}

A Brain-Machine-Brain-Interface (BMBI) is a sophisticated communication pathway where electronic signal interexchange takes place between the neurons of a living being and a machine/computer. It's also known as Brain-Computer-Interface (BCI). In general, in a BMBI system, an electrode is implanted in a specific region of the brain, and carries the signal from the brain and sends it out to the computer. A computer analyzes the signal and generates responses and sends it back to the brain. These exchanges of signals propagate via a wired/wireless connection established between the brain and the machine. BMBI is first brought to light by a group of researchers in the University of California Los Angeles (UCLA) in 1970 working under the project funded by the National Science

Foundation (NSF) and DARPA [1]. Since then, the BMBI system is evolving through a tremendous amount of research and development to make it a viable, power efficient, user friendly, matured technology [2-13]. Attention is paid in every direction of technological development, i.e. innovation is brought into the electrode and electrode system design for achieving improved electrode-tissue interface and efficient neural recording [3-5]. Different advance data processing and wireless communication modules are being designed for improved data analysis and efficient exchange of information between the brain and the off-body machine $[6,10]$. Architectural and operational development are taking place to make it a user friendly device $[4,11]$ so that it could be carried as a suitable body implant. Noticeable attention is paid to make the BMBI system energy sustainable $[12,13]$. In ideal situation, it should be battery less system which would be able to run by the harvested power from the ambience. Development of cloud computation is adding 
another dimension to the development and proliferation of BMBI technology. Integration of facilities enabling cloud computation with the existing BMBI system is making it a more intelligent and smarter technology. In a Smart BMBI system electrodes send data to the

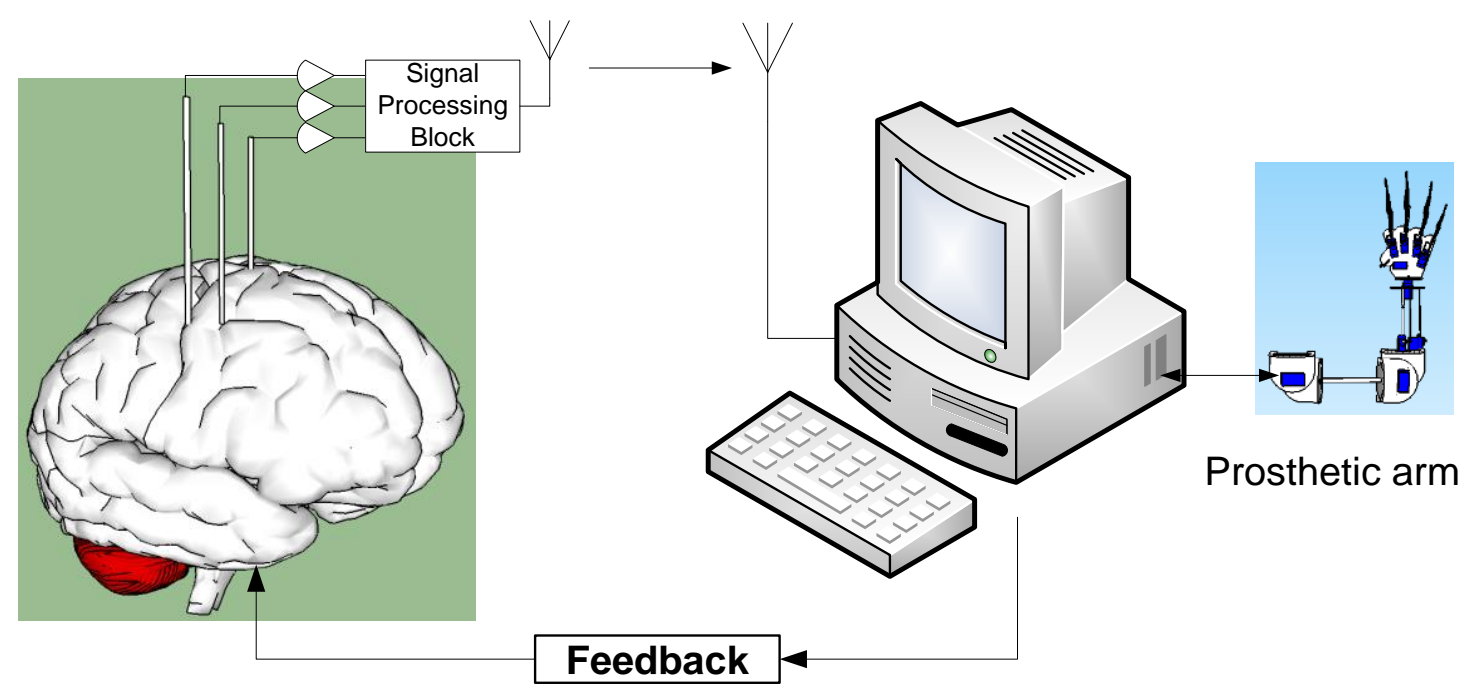

Figure 1: A traditional Brain-Machine-Brain-Interface system.

backend computer, and the computer sends it to a remote sever to take advantage of the powerful cloud computation. Fig 1 shows a basic structure of BMBI system.

\subsection{BMBI in Deep Brain Stimulation (DBS)}

A very popular example of brain machine interface is the Deep Brain Stimulation (DBS) system, where an implanted electrode stimulates a specific area of the brain to treat movement disorder including Parkinson's disease, essential tremor and dystonia. The gradual deterioration of the dopaminergic neuron [14] in the area known as substantia nigra pars compacta $(\mathrm{SNc})$ inside the brain that generates dopamine, a neurotransmitter which maintains the balance of the stimulation flow in the basal ganglia-thalamo-cortical loop via 
the receptor D1-5 [15], has been strongly argued as the cause of movement disorder syndrome. A proper level of Dopamine excites the striatal neurons in the direct pathway and inhibits the striatal neurons in the indirect pathway thereby facilitating smooth movement in vivo. Scarcity or abundance of Dopamine both can cause the lack of coordination in movement, which is pathologically classified as different types of movement disorders. Depending on their different clinical symptoms these kinds of

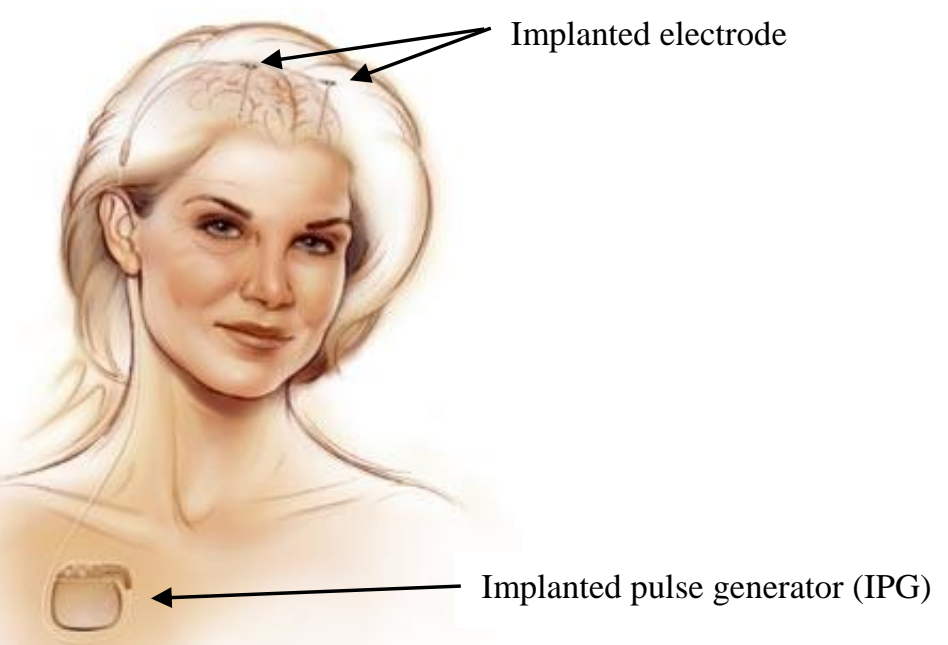

Figure 2: Schematic of a Deep Brain Stimulation system [16]

disorders are named as Parkinson disease, essential tremor, epilepsy, dystonia etc. Nearly 50 million of world population is suffering from these kinds of diseases. As a medicinal treatment of these syndromes, dopaminergic drugs or botulinum toxin injection therapy (BOTOX® therapy) have been used for long time. Even though these medicinal treatment help producing the dopamine release in the SNc in the brain, the chronic use of it makes the brain cell irresponsive to the drug or sometime, due to the uncontrolled use of drugs, the excessive secretion of dopamine causes other type of Basal Ganglia-thalamo-cortical 
dysfunction including other side effects like dizziness, emotion disorder, etc. [14]. Recent study shows that about $40 \%$ of the total population suffering from different types of movement disorders is irresponsive or somehow incapacitate to the medicinal therapy [17]. For treating this kind of patients, due to its reversibility and adjustability, the Deep Brain Stimulation (DBS) technique has been a proven therapeutic alternative to the surgical incision (i.e. thalamotomy, pallidotomy) of the certain brain parts [15, 17]. Fig 2 shows a typical DBS system.

In DBS, an electrode is implanted inside the deeper brain with an objective to deliver precise electrical stimulation to the dysfunctional regions, most preferably the STN and/or GPi region of the Basal ganglia circuitry. It is suggested that the high frequency stimulation with specific parameters — wave shape, amplitude, frequency, pulse width, duration—from the electrode has potential to disrupt the abnormal synchrony in the oscillation in the Basalganglia circuitry that causes the involuntary movement. It works as a jammer for the burst signal in the LFP of neuro-stimulation to circulate through the network. However, the complete mechanism of how DBS works is yet to be unfolded-any empirical or theoretical model explaining MD has not been claimed to date. Due to the lack of complete and proven knowledge of neurophysiology of movement disorder and the action of deep brain stimulation over the basal-ganglia feedback loop, the preferable set of waveform and its associate parameters for a certain patient is a subject of personalization. Owing to this fact, to take the advantage of DBS in treating movement disorder a highly modular DBS system, where the stimulation wave parameters, (i.e. shape, frequency, amplitude, 
stimulation duration, etc.) can be easily modified according to the individual patient's needs, is more than desirable.

\subsection{Challenges in Current DBS systems}

\subsubsection{Lack of Smartness}

Current DBS devices severely lack in smartness - they don't have ability to get connected with the cloud. Without getting connected with the internet, it is not being able to take the advantage of powerful cloud computation in data analysis and decision making. In addition, due to the lack of connectivity, remote access and monitoring of the patient is also not being possible. Next generation DBS should overcome this drawback.

\subsubsection{Energy Constraint}

Current DBS system uses an internal battery for the operation of neuro-stimulator [18]. It utilizes the battery power for internal processing, stimulation generation and off-body communication. Due to the use of battery, the neuro-stimulator is subjected to periodic maintenance which requires surgical intervention. In the survey of recent research progression, it is being observed that contemporary research is putting a tremendous effort to overcome this limitation [19-25]. Current research is focusing on implementation of different wireless charge transfer mechanism to recharge the internal battery. Amongst the recent reported techniques, magnetic coupled charge transfer technique, which requires implanting an additional magnetic coil inside the body to couple the charge from the offbody primary coil, has gained attention. Even though this system exhibits a considerable potential for recharging the internal battery, unfortunately, it suffers from numerous 
negative side effects associated with poor human tissue-material contact. Most common side effect includes inflammation, infection, swelling in the implanting region due to the corrosive byproduct generated from the gradual erosion of the metal coil, lethargy of the associated body tissue due to the continuous heating of the coil and so on. In addition, the required size of the implanted coil is another issue. As a potential solution to this problem, we strongly argue that, instead of inductively coupled charge transfer mechanism, a wireless far field energy harvesting network would be a better choice. In the later section of this dissertation we have demonstrated a far-field wireless harvesting circuitry for a smart deep brain stimulation system.

\subsubsection{Power Efficient Communication Backbone}

To establish the communication from brain to machine and machine to brain, which we rhetorically name as Brain-Machine-Brain (BMB) communication, a suitable, power efficient communication backbone plays an important role in a viable DBS system. To date reported DBS systems have been using mostly active communication technology which is subjected to requiring local oscillator, complex modulation and demodulation circuitry for enabling bi-directional wireless communication to the backend controller. Recent technological development has used different standard or nonstandard technologies (i.e. ZigBee, Bluetooth, UWB communication, etc.) over the ISM band. However to realize the next generation energy efficient DBS system, instead of using active communication technology, a passive, bi-directional communication backbone would be a better choice. We strongly argue that, considering its fully passive communication feature, less resourceful communication hardware requirement, and already existing in-chip fabrication 
facilities, a RFID commutation backbone based on EPC Gen2 standard air interface protocol would offer a suitable choice for the next generation DBS system. However to use this existing RFID platform in the DBS system, a tremendous technological modification is required to redefine and redesign its protocol standard to make it carry the non-EPC standard data and command. Chapter II of this dissertation demonstrates a reconfigurable sensing platform based on RFID Gen-2 protocol, where the redefinition and modification of existing RFID protocols has been thoroughly explained.

\subsubsection{Data Security Issue}

Protecting individual's privacy is another burning issue in any kind of human implantable devices. DBS is not different from that. Current DBS system implements a very minimal or no security to protect the data. It's very much vulnerable to data eavesdropping, traffic analysis, spoofing or denial of service attacks and data manipulation by any malicious

device or person. In addition, current DBS systems like Medtronic ActivaPc [26], does not have any kind of mechanism for user authentication and verification, which brings up risks of unauthorized access and modification of intelligence of the system. Addressing these security issues would be one of the prime challenges for the next generation Smart DBS system.

\subsubsection{Limitation in Stimulation Waveforms}

Machine to Brain interaction is one of the important features of DBS. External electrical stimulation possesses a significant potential to alter the Brain's neuro-activities. However, the complete mechanism of how DBS works is yet to be unfolded. To study the brain 
activity and enrich the current knowledge about brain's response to the electrical stimulation, the best machine-to-brain interface (MBI) should be capable of providing a wide variety of electronic inputs or waveforms to any targeted neural areas. The current systems, to date reported [18-20], has limited capability: they can only generate few preset wave-shapes of known type. However, the effect of the other side of the wave spectrumthe arbitrary wave - is totally unfolded. To enrich the current knowledge, it is important to examine the effect of arbitrary wave over the neural activities. This research aims to investigate the generation, incorporation and optimization of the arbitrary stimulating waves along with a wide variety of preset waves, with the capability to modify the basic wave parameters, i.e. amplitude, frequency, pulse width and pulse duration, dynamically.

\subsubsection{Constraint in True Real-time Neuro Recording}

For realizing an effective Brain-Machine-Brain interface (BMBI), monitoring and analysis of brain activities is of significance importance. To date, for deep brain recording, the reported system employed single control [18] or coordinated control [19-21] devices for neural recording. In those systems, the electrode is just a passive conductor for those neural signals; all the processing — filtering, amplification and digitization — is performed inside the controller [21, 33]. But our investigation of the current DBS system has identified few drawbacks: firstly the controller being placed relatively away from the recording site (i.e. in the single control system like Medtronic 3387 or 3389, the controller is placed somewhere in the chest or abdomen [18]; in the recent reported system [27-33], it is placed somewhere closer to the bur hole) the signal has to travel a longer path which is subjected to resistive losses; and secondly, in a multisite recording system, where a bunch of 
electrodes are being controlled by a single controller, real-time recording and parallel processing of the neural activity is not possible. Our research strongly argues that by integrating an on-site, autonomous neuro-recorder in the electrode and implanting multiple electrodes in different sites-if necessary-these limitations can be overcome. Implementation of the on-site recording channel inside the electrode requires careful designing and integration of the low pass filters, Low Noise Amplifier (LNA) and highly sensitive, low power Analog to Digital converters. In chapter IV, we have demonstrated an autonomous, smart electrode for Deep Brain Stimulation, where these issues have been thoroughly discussed and provable solutions have been presented.

\subsection{Next Generation Smart Deep Brain Stimulator (SDBS)}

To address the limitations of current DBS system, in this research, we have designed and implemented an innovative Smart Deep Brain Stimulator (SDBS) as an application of SBMBI. It consists of brain-implantable smart electrodes and a wireless-connected external controller. The SDBS electrodes operate as completely autonomous electronic implants that are capable of sensing and recording neural activities in real time, performing local processing, and generating arbitrary waveforms for neuro- stimulation. A bidirectional, secure, fully-passive wireless communication backbone was designed and integrated into this smart electrode to maintain contact between the smart electrodes and the controller. The standard EPC-Global protocol has been modified and adopted as the communication protocol in this design. The proposed SDBS was demonstrated and tested through a hardware prototype. 


\subsection{Low Power Wireless ECG Sensing}

\subsubsection{Electro Cardiogram (ECG)}

Electro Cardiogram is an electronic signature that provides vital information of the cardiovascular system. It is a painless, harmless, non-invasive way of tracking the heart activities, and works as a very popular diagnostic tool for cardiovascular diseases like,

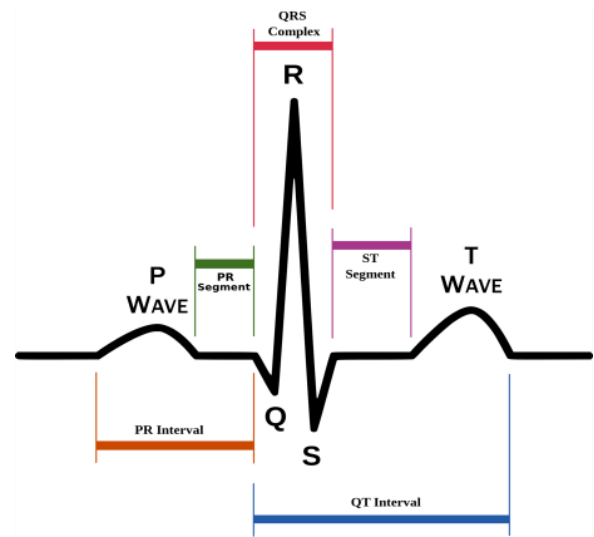

Figure 3: A standard ECG signal with "PQRST" complex [34]

cardiac arrhythmia, heart attack, heart failure, etc. Fig 3 shows a typical ECG pulse from a healthy heart. As technically named, it is composed of P, Q, R, S and T complexes. The first upward pulse of the signal, the P complex, is formed when the atria (the two upper chamber of the heart) contracts to pump the blood into the ventricles (the two lower chambers of the heart). The next large segment, the QRS complex, is formed when the ventricles contract to pump blood out of it. The following segment, the ST segment, measures the end of the contraction of the ventricles to the beginning of the rest period before the ventricles begin to contract for the next beat. The next rising section, the $\mathrm{T}$ complex, demonstrates the resting period of the ventricles. Comparing individual's PQRST complex and its train with the standard QPRST train helps diagnosing cardiac diseases. 


\subsubsection{Wireless Cardiac Activity Monitoring}

Cardiac arrest is one of the leading causes of human mortality around the world [35]. However this risk from cardiac malfunctioning can be greatly reduced if it is detected earlier. Even though cardiac malfunctioning does not precede with that much of noticeable signs, continuous monitoring and analysis of the patient's pulmonary activities monitoring through any standard Electro Cardiogram (ECG) device has potential to reduce the risk in many folds [36]. Continuous monitoring of the patient's pulmonary signal can generate alarm and helps the care professionals to take necessary steps thereby improving patient care in cardiovascular diseases. However a suitable, efficient system day to day outpatients' use is yet to be found.

\subsection{Research Objective}

In this research, we have designed and developed a smart platform for Brain-Machin-Brain Interface (SBMBI), and studied its feasibility to configure it as a next generation Smart Deep Brain Stimulator (SDBS) which would be able to address the limitations encountered in the existing systems. In addition, SBMBI platform has been tested for its applicability in other bio-medical applications, as well. In the later part of this thesis, using the SBMBI as a sensing platform, a low power, wireless Electro Cardiogram (ECG) monitoring device has been demonstrated. This device records heart's activities, analyzes it locally, and also sends it to a high-end remote server for rigorous analysis. In vivo test is performed to test the integrity of the system performance. 
CHAPTER II

RECONFIGURABLE SMART COMMUNICATION PLATFORM 


\subsection{Introduction:}

This chapter introduces a semi-passive, re-configurable UHF RFID sensing tag operating as the Generic Sensing Platform (GSP) and the sensing nodes of the future Machine to Machine (M2M) communication. For generic real-time data acquisition, this new platform offers multiple inexpensive, plug-and-play sensing channels for the appended physical sensors. This new tag can be dynamically reconfigured to operate as a continuous data transmitting/receiving platform (online mode) or a data logging device (offline mode). In the offline mode, for accommodating and managing the high volume of data acquired by the tag sensors, a novel memory management scheme is designed and implemented. Furthermore, the platform employs an innovative data communication strategy, compatible with the EPC Gen-2 protocol standard, to facilitate communication between the sensing node and the cloud via a smart backend controller. An experimental Internet of Things (IoT) test bench is staged to validate the performance of the design platform.

Internet of Things (IoT) is considered to be the paradigm of the next-generation networking platform in machine-to-machine (M2M) communications [37]. The sensor nodes/platforms, which empower the objects that the sensors are attached to with a digital identifier, the real-time sensing capability and the access to any other nodes or remote resources in the network, are the most crucial part of the future IoT [37]-[43]. Fig. 4 shows the architecture of the envisioned IoT consisting of three-tier communications, i.e. local, regional and global tiers. The first tier is wireless sensing nodes/platforms. They are equipped with multiple sensors of various nature, and may form an ad-hoc network with the other local nodes and can acquire intelligence or raw data and send them to the next 
tier, i.e. the regional base station tier, through the ISM band radio link (IEEE 802.15.4 standard) and light weight protocol stack (i.e. 6LoWPAN, RFID). The base station works as the internet gateway between the sensor node tier and the cloud (i.e. Ethernet, IEEE 802.11, 4G/LTE infrastructure) and routs the raw sensing data to another node or a remote server through the cloud over the standard protocol stack (i.e. CoAP, TCP/IP, UDP) [38], [42].

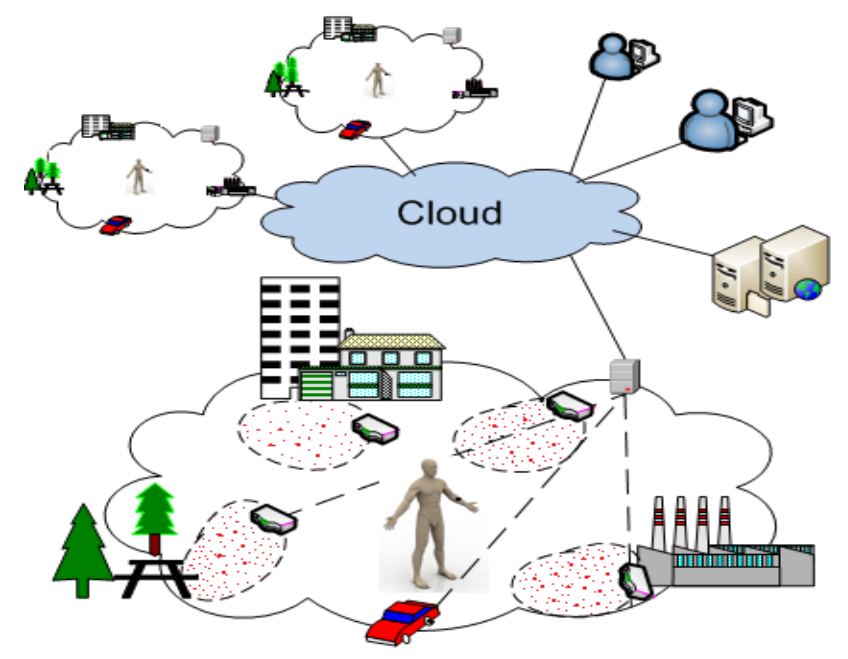

Figure 4: The architecture of the envisioned three-tier IoT

With the recent advances in sensing, cloud computing and wireless communications technologies, IoT is envisioned to be the future global networking framework of physical/contextual phenomena for all objects [43]. For example, these phenomena could be related to the context-awareness parameters in a smart home environment $[44,45]$, security status [41], healthcare and biometric identification process [46], [47], climate and environmental variables [48], and etc. They could be a heterogeneous combination of different classes depending on a very dynamic set of choices of the users [43]. Due to the 
huge diversity in the types $\&$ the classes of the acquirable intelligence, IoT is literally an open ended cyber-physical network where the sensing scope for the embedded sensor nodes/platforms is scalable and unlimited. Therefore, inexpensive adaptable devices used as the sensing nodes in the system are the most critical parts of the future IoT. Custom design and manufacturing of all sensor nodes used in the IoT is impractical and unnecessary. Instead a generic sensing platform (GSP) that is adaptable for all possible IoT sensing needs should be designed developed and mass-produced as the general IoT sensing nodes.

In addition to the diversity in the nature of the information to be acquired, the sensing nodes need to accommodate the data acquisition and transmission pattern that is a function of a few closely related variables such as application requirements, available resources and overhead cost of the network. But it is primarily determined by the application requirements. To find out a suitable set of solutions for the data acquisition, we have divided this diverse realm of the data sensing applications into two broad classes: (i) the sensing applications where continuous acquisition and sending the sensor data to the backend server or higher-tier systems is required and the nodes operate in online mode (i.e. postoperative patient care system [49]); and (ii) the ones where periodic acquisition and logging of the sensed data into the local memory stored for transmission later on while working in offline mode [48]. This periodicity of data acquisition and transmission could also vary significantly from system to system. For IoT, unless required otherwise, data logging operation mode is mostly preferred-because, in this case the intermittent usage of the channel resources would significantly reduce the network overhead cost. However, 
in some sensing scenarios, this overhead cost could become a less important issue. For example, in a post-traumatic patient care IoT system, instantaneous transmission of the monitoring data to the backend server for detailed analysis and diagnosis should be put in higher priority over the overhead cost [49].

Therefore, IoT system design is always subject to a tradeoff between the cost and its performance. The system design engineers have to make the balanced choice of suitable sensing platforms for a particular IoT application. In addition, sometimes finding the right kind of off-the-shelf sensing platforms/devices for an IoT system is very challenging and even impossible. However, the design cost would be minimized if re-configurable and efficient (in term of the power, size and communication protocol compatibility) generic sensor node/platforms are available for IoT design. To accommodate the rapid growth of future IoT, we must address the design issue of the sensing node/platforms.

Technically there are two general classes of possible sensing platforms for IoT applications, i.e. wireless sensor nodes (WSN) and RFID tag-based sensing platforms. Wireless sensor nodes generally offer strong computational capability. Some of the wireless sensor nodes such as Mica2 and iMote are equipped with generic sensing capabilities, but they are severely disadvantageous in operating power, physical size and the manufacturing cost [50], which may pose a major threat to the materialization of the next generation ubiquitous sensing and pervasive networking in IoT.

On the other hand, RFID sensing tags could also be used as the sensing nodes in IoT applications [52]-[54] for their enhanced sensorial capabilities along with the tiny size and 
very cost-effective RF-based identification mechanism, for which they have been known to the world for decades.

However, the acceptability of RFID platforms towards the sensing nodes in IoT architecture was sometimes deemed off-the-mark and very critically viewed due to some inherent limitations associated with the existing RFID tag design [54], [55]. To date, the existing RFID platforms implemented in the IoT are mostly passive, i.e. they cannot operate or sense data without being laid inside the reader's reading zone. In addition, current sensor RFID tags usually come up with single sensor [56], [57] or, in some cases, with multiple sensors [57] built-in with it. But once they are manufactured, they cannot be replaced or reconfigured without costly redesigning and reproduction. Since IoT is an open, dynamic and versatile global networking and sensing system in nature, the generality, modularity or re-configurability of the sensing nodes/platform is essential in the adoption of the sensing nodes/platforms in the future IoT architecture.

This article presents an approach of designing Gen-2 compatible, semi-passive RFID generic sensing platforms (GSP-tag) as the sensing nodes of the future IoT. To facilitate sensor modularity and generic data acquisition in the RFID tag design, we have embedded the features of plug-n-play, sensor variety compatibility, and multiple sensing channels into the new tag design. This tag also features dynamic re-configurability, and can be switched to run as (i) a continuous data transmission platform (online use), or (ii) a data logging platform (offline use). In addition to the basic platform re-configurability, the resolution of the analog-digital converter (ADC) in each sensing channel can also be dynamically adjusted to accommodate different requirements for the design of various IoT systems. 
Especially, in the data logging mode, a proper tradeoff can be made between the total operation time and the available memory resources of the GSP. The GSP-tag can further be tuned to set the desired data acquisition frequency, which would be useful for energy saving in offline mode by selecting a lower data acquisition frequency for a relatively longer period of deployment time.

The design of this new generic sensing tag will provide the IoT designers a flexible way to choose the right kind of sensing platforms for their particular applications and to smoothly incorporate them with the existing IoT infrastructure. Moreover, the new tag would provide the sensor application developers with an open platform for almost any kind of sensor RFID application development. Commercial implementation of this GSP-tag would be beneficial to improving IoT manufacturing efficiency and reducing the production costs accordingly. With the huge versatility in sensing capability and cost effectiveness, we expect this GSP-tag will be a very popular choice for designing and implementing the sensor nodes in the increasingly popular future Internet of Things (IoT).

\subsection{Related Work}

Ever since the emergence of the field of IoT, IoT and RFID have been mutually facilitating each other technically. Among the manifold technical progress directions for RFID, there has been growing interest in equipping the existing tags with higher sensorial capability. In recent years, passive sensor RFID tags [54], and even sometimes, chip-less RFID sensing platforms [58] have been very popular choices in RFID tag design for their power efficient nature. However, this type of sensing platform is has to be accompanied by a RFID reader to be able to operate and sense. 
The uninterrupted data sensing and logging issue for the RFID sensing platforms out of the reader's proximity, has been identified in a plethora of research projects around the world. For example, Deniel J. Yeager et al presented an approach using a wirelessly powered passive data logger [59]. Once the internal battery of the logger is fully charged, it can monitor and $\log$ the data for about 24 hours. However, for most of the data logging applications such as supply chain monitoring and remote environmental sensing, having a longer period of uninterrupted data-logging operation -time is advantageous and preferred. In a separate research, Tomas Unander et al presented a battery-assisted 2-chip data logger dealing with this issue of limited operation time [60]. This tag can sustain operations for years without changing battery. This design scheme has literally made the operation-time limitation a nonissue for the tag applications in supply chain and other such applications requiring a long data logging period as military logistic [61] and surveillance [62], environmental monitoring [63], and remote health monitoring service [64]. However, in that RFID design, including an extra chip for data acquisition and logging only in the existing RFID systems will add up to the cost of the tags significantly. For IoT applications where millions and millions of tag data loggers may be needed yearly, the extra costs incurred may be unbearable. Moreover, this article did not address how a large volume of data was handled with the limited size of the user memory block if the tag was deployed for a long period of time (i.e. 528-bit memory for typical Gen2 tags [65]).

Besides the data logging function, the continuous data transmission feature of sensing tags is equally intriguing and important in RFID and IoT applications. For example, in a pharmaceutical drug delivery chain, a continuous monitoring and forecasting system of the 
containers' temperature might save millions of dollars from supply chain losses [66]. Fortunately, RFID sensing platforms offer a very cost effective way dealing with that kind of problems. In a pilot partnership program launched by DHL and AeroScout as a response to the incident taken place in 2006, where 20,000 doses of Novartis' Flu vaccines were accidentally frozen to waste, Wi-Fi enabled active RFID tags with temperature sensors were placed inside containers filled with pharmaceutical products to have them visually monitored in real-time based on the ambient temperature [67]. The pilot program has been

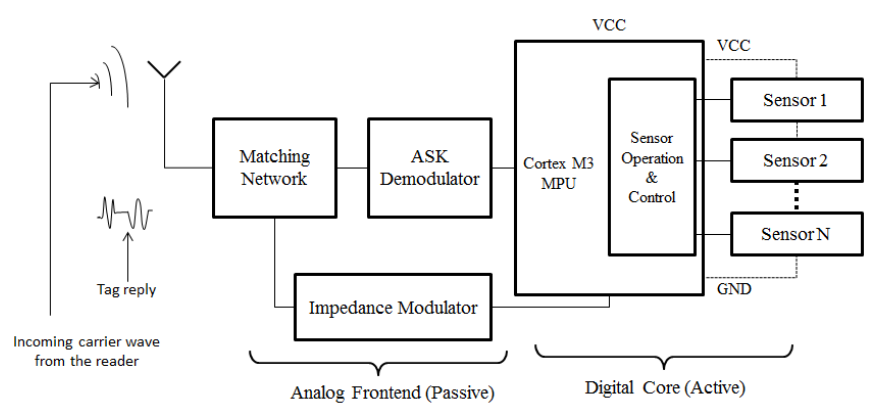

(a)

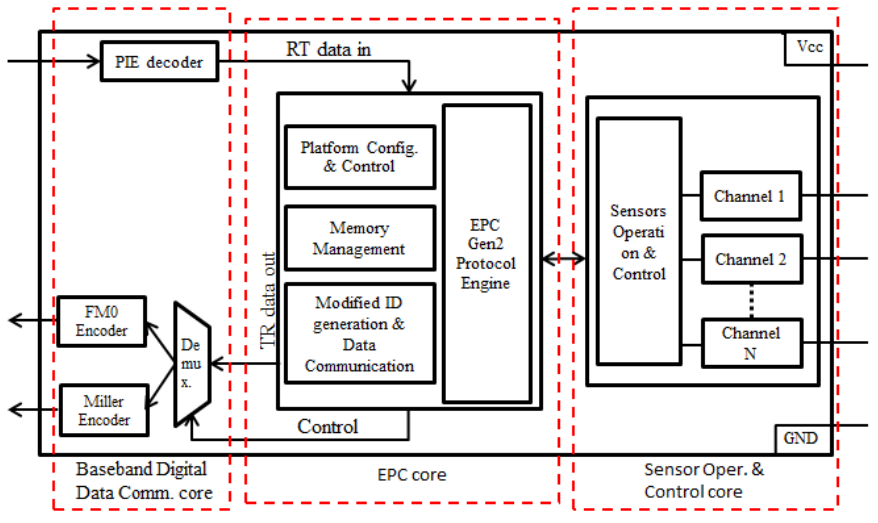

(b)

Figure 5: (a) Schematic block diagram of the GSP-tag. (b) Second level block diagram of the software defined Digital core, implemented inside a Cortex M3 Microprocessor.

reported to be successful in achieving the real-time visibility and monitoring of the shipments. The result from the program suggested high market demands for sensing RFID tags, which, once again, reflected the same on-going demand for power-efficient and cost- 
effective passive or semi-passive RFID solutions. The study conducted by Alanson P. Sample et al further shows that a passive RFID sensing platform can sense and send data to the back end reader continuously [68]. Unfortunately, the reported passive sensing tags are EPC GEN-1 compatible only, whereas almost all of the existing market infrastructures are based on EPC GEN-2 standard. Therefore, the design of GEN2-compatible dynamically reconfigurable generic RFID sensing platforms suitable for IoT applications had remained to an unsolved issue prior to this work.

\subsection{Design Methodology}

The designed GSP-tag has been divided into two basic building blocks: (i) the analog frontend and (ii) the digital core block, as shown in Fig. 5. The analog frontend includes the analog modulation and demodulation circuitry along with the antenna matching network. The Digital core handles the operation of the baseband digital data communication, the implementation of EPC Gen2 protocols as well as the sensor operation and control.

The digital core part of the tag is fully software-defined. Programming using a combination of $\mathrm{C}$ and the Assembly languages are employed to implement the digital core functions. Specifically, the baseband digital data communications (i.e. encoders and decoders), in which timing is critical, are implemented using the assembly language. The rest of the cores have been programed using $\mathrm{C}$ language, due to its more user friendly framework. The EPC core includes the EPC Gen2 protocol engine as well as the tag platform configuration block, memory management algorithm and modified EPC ID generation and sensor data 
communication block. The key to the design of the sensor operation \& control core basically is the modeling and design of the generic data acquisition channels for the platform.

Using the multi-threaded programing approach, we have designed and made the tag and reader communications and the sensor operation two separate and independent processes within a single chip. The programming approach enabled the tag to perform data acquisition and processing independently without interrupting the main task that performs the digital baseband data communications and drives the EPC Gen2 protocol engine.

For regular RFID operations, a tag transmits the EPC ID continuously when it is in the reader's interrogation range. Although not exactly continuous in a mathematical sense, the transmission from a sensing platform with the minimal time gap between two transmissions of about 300 $\mu$ s (in best case scenario) [69] can be considered to continuous [58], [59], [70]. In our design, to accommodate a larger data field, we have used SSCC 96bit protocol standard [71] as the primary medium of communications between the sensing nodes and the back-end server.

Since this tag is designed to operate as a generic sensing platform (GSP-tag), the channels are modeled and designed in such a way that, to the GSP's data acquisition and the postprocessing engine, they must be sensor-type independent. In another word, to the channels, the sensors are viewed as nothing but a voltage source where, the application software running at the back end server interprets the data correctly with the help of the corresponding calibration curves of the sensors. The reference voltage of the ADCs in our 
design is set to be $2.5 \mathrm{~V}$, which is the upper voltage limit that can be acquired using these channels. Thus GSP can sense and store any voltage levels between 0 to $2500 \mathrm{mV}$, with the resolution of the data actuation dependent on the actual resolution of the ADC and the sensitivity of the channels. The design allows the tag users to choose the resolution of the ADC to be one of three modes-12-bit, 10-bit and 8-bit-by writing the 4th hex location (Fig. $8 \&$ Table I) of the 1 st memory block in the User memory location reserved for the system configuration. With the 12-bit mode set, even though the resolution allows us to measure voltage difference down to $0.649 \mathrm{mv}$, the maximum sensitivity of the channels is $1.00 \mathrm{mv}$ and can represent totally 2500 different voltage levels between $0-2.5 \mathrm{v}$. Hence, any valid data that are reported by the GSP-tag is represented with a hex value within the range of $0 \mathrm{x} 0000$ to $0 \mathrm{x} 09 \mathrm{C} 4$, and data values above $0 \times 09 \mathrm{C} 4$ are considered to be invalid.

\subsection{System Framework and Implementation}

This section describes the design and implementation of the GSP-tag as outlined in the previous section. To make this section concise, we will emphasize the following technical aspects:

- Tag Platform re-configurability

- Implementation of memory management algorithm

- EPC ID modification and Data communication

- Power and battery management 
The GSP tag is designed to work as a semi-passive device. Its digital core is batteryassisted; whereas the analog frontend is completely passive in design. The analog frontend of the GSP-tag is comprised of a $915 \mathrm{MHz}$ meandering antenna followed by the L-C

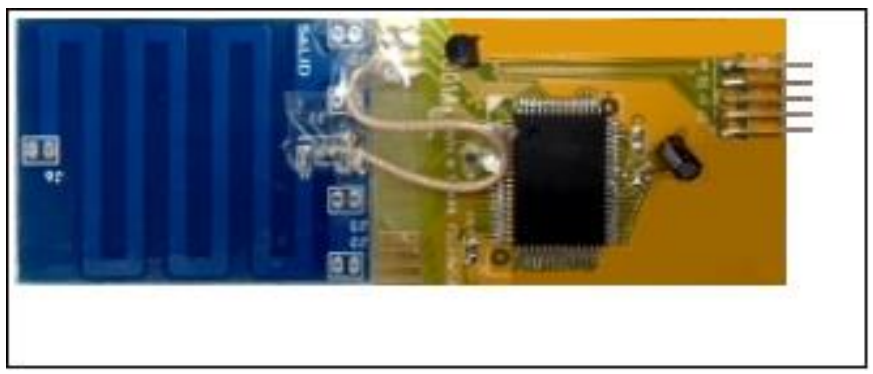

Figure 6: Implemented GSP-tag on a PCB with the off-the-shelf components

matching network and modulation-demodulation circuitry. Since the analog frontend is completely passive, the operation of the modulation/demodulation depends on the reader's

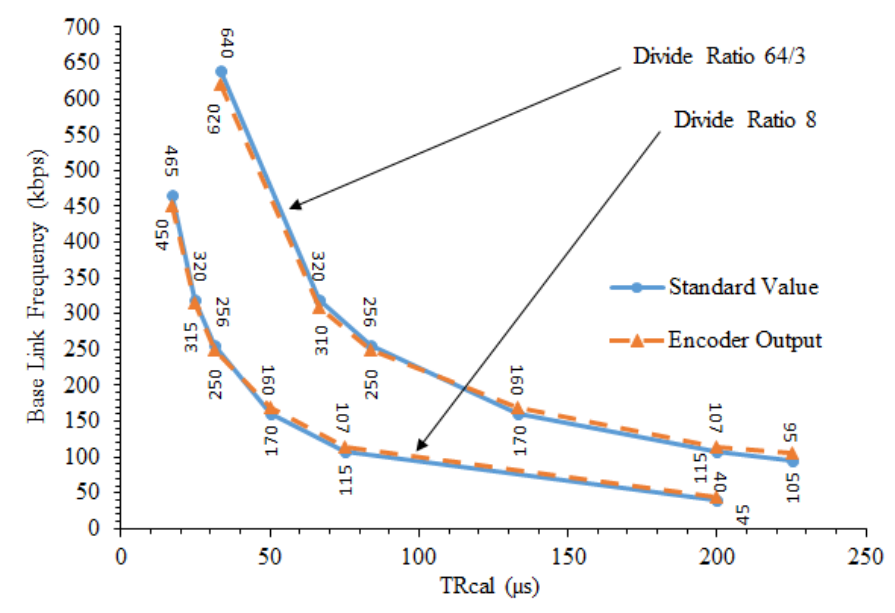

Figure 7: BLF generation performance curve of the encoders

radiated power. The data modulation from the tag to the reader is achieved by changing the impedance of the antenna matching network based on the digital data values. In our design we have used 2N7000, a high speed MOSFET with a switching speed of 7ns [72], 
TABLE I

Tag Configuration Setup

\begin{tabular}{|c|c|c|c|c|}
\hline $\begin{array}{l}\text { Configuration } \\
\text { Name }\end{array}$ & $\begin{array}{l}1^{\text {st }} \text { Memory } \\
\text { Block }\end{array}$ & $\begin{array}{l}2^{\text {nd }} \text { Memory } \\
\text { Block }\end{array}$ & $\begin{array}{l}3^{\text {rd }} \text { Memory } \\
\text { Block }\end{array}$ & Comments \\
\hline $\begin{array}{l}\text { Continuous mode } \\
\text { switching }\end{array}$ & 0x0000 & -- & -- & $\begin{array}{l}0 \times X 000 \text { switches it to the } \\
\text { continuous mode and } O x O X X X \\
\text { sets the resolution of the ADC }\end{array}$ \\
\hline $\begin{array}{l}\text { Data logging mode } \\
\text { switching }\end{array}$ & 0x0FFF & $\begin{array}{l}\text { 0x0001- } \\
\text { 0xFFFF }\end{array}$ & -- & $\begin{array}{l}1^{\text {st }} \text { memory block: } 0 x O X X X \\
\text { represent the resolution of the } \\
\text { ADC and } O x X F F F \text { switches it to } \\
\text { the data logging mode. }\end{array}$ \\
\hline & & & & $\begin{array}{l}2^{\text {nd }} \text { memory block: sets the data } \\
\text { fetching interval time (in ' } s \text { ') }\end{array}$ \\
\hline Memory reset & -- & -- & 0x0000 & $\begin{array}{l}\text { Reset all of the available user } \\
\text { memory blocks with a value of } \\
O x F F F F \text { except } 1^{\text {st }} \text { and } 2^{\text {nd }} \\
\text { blocks }\end{array}$ \\
\hline
\end{tabular}

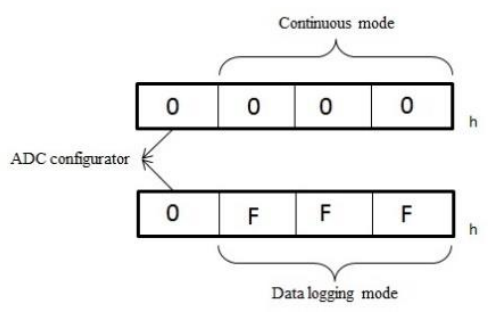

Figure 8: Schematic diagram of the 1st configuration block inside the used memory location

to modulate the $915 \mathrm{MHz}$ carrier with the data rate of up to $640 \mathrm{KHz}$. In addition, we employed a low power Shottky diode (HMS 285x) to implement the ASK demodulator. Fig. 6 shows the snap shot of the implemented GSP tag.

The digital core, which is fully software-defined, is implemented through a Cortex M3 microcontroller. It performs digital baseband data communication and enforces the EPC protocols along with other advanced tag features. With the EPC Gen2 protocol used in the design, the tag must incorporate the PI (Pulse Interval) decoding and Miller and FM0 encoding techniques [69]. While an edge triggered interrupt mechanism was enough to 
decode the incoming data, the real challenge for the software design was to generate the proper bit rates/Base Link Frequency (BLF) throughput from these encoders. In addition, the tag encoders must support variable BLF, between $40 \mathrm{KHz}$ and $640 \mathrm{kHz}$. Eqn. 1 shows the relation between TRcal and the Divide ratio (DR), which is provided by the reader directly at the very beginning of each session. To maintain this strict timing requirement, the assembly language was used to program the system to get the direct control over the MPU registers and to avoid extra instruction execution delay. Fig. 7 shows the performance of the designed encoders.

$$
B L F=(T R c a l / D R)
$$

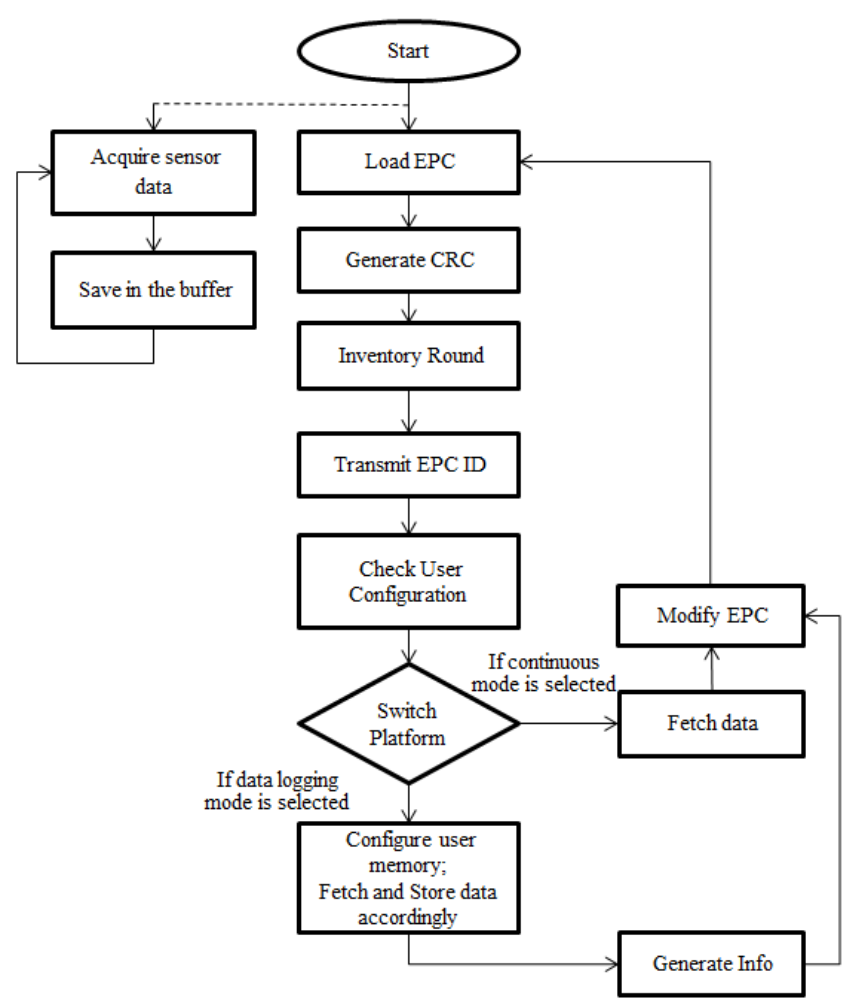

Figure 9: Firmware state diagram of the GSP's Digital core 
TABLE II

GSP-Tag ID Modification Table

\begin{tabular}{|c|c|c|}
\hline Event Name & Reported value at the variable data field (36 bit) & Platform mode \\
\hline $\begin{array}{l}\text { Channels' instant data } \\
\text { value }\end{array}$ & $\begin{array}{l}\text { The entire variable data field is updated according } \\
\text { to the instant channels' data, where a possible } \\
\text { value might take any value from 0x000000000 to } \\
\text { 0x9C49C49C4 }\end{array}$ & $\begin{array}{l}\text { Continuous data } \\
\text { transmitting mode }\end{array}$ \\
\hline $\begin{array}{l}0^{\text {th }} \text { instance of missed } \\
\text { logging }\end{array}$ & 0xA00A00A00 & \\
\hline Memory filled warning & 0xFFFFFFFFF & \\
\hline $\begin{array}{l}1^{\text {st }} \text { instance of missed } \\
\text { logging }\end{array}$ & 0xA01A01A01 & Data logging mode \\
\hline$"$ & , & \\
\hline $\begin{array}{l}255^{\text {th }} \text { instance of missed } \\
\text { logging }\end{array}$ & 0xAFFAFFAFF & \\
\hline
\end{tabular}

To enable this tag with the advanced features like re-configurability, efficient memory management and the ID communication capability, the EPC Gen2 protocol has been craftily modified so that it does not lose its compatibility with the Gen2 infrastructure. Fig. 9 shows the basic flowchart of the developed firmware.

\subsubsection{Tag Platform Configuration}

The GSP-tag can be dynamically switched between two primary modes of operation: 1) Data logging mode and 2) Continuous data transmitting mode. In addition, the data acquisition frequency and the resolution of the ADC can also be reconfigured. To enable this tag with re-configurability, first three memory units ( 1 unit $=16$ bit of memory) of the User memory block have been reserved as the tag configuration setup block. The design allows a conventional Gen2 write command (i.e. G2Write $=3,0,0 \mathrm{~F} F$ ) to set the configuration parameters. While executing the normal functions, the EPC core periodically 
checks the configuration blocks to get the updated modes of the operation and memory management and to reset it accordingly.

At the start up the tag registers itself to the reader with the default EPC ID (tag transmits its reduced unique ID code along with the data fields (Fig. 11c) filled with all zeroes). After the tag is successfully registered, it is ready to go to the Access State, where it accepts all the sets of Gen2 Read \& Write commands to modify its 'User configuration' location. After

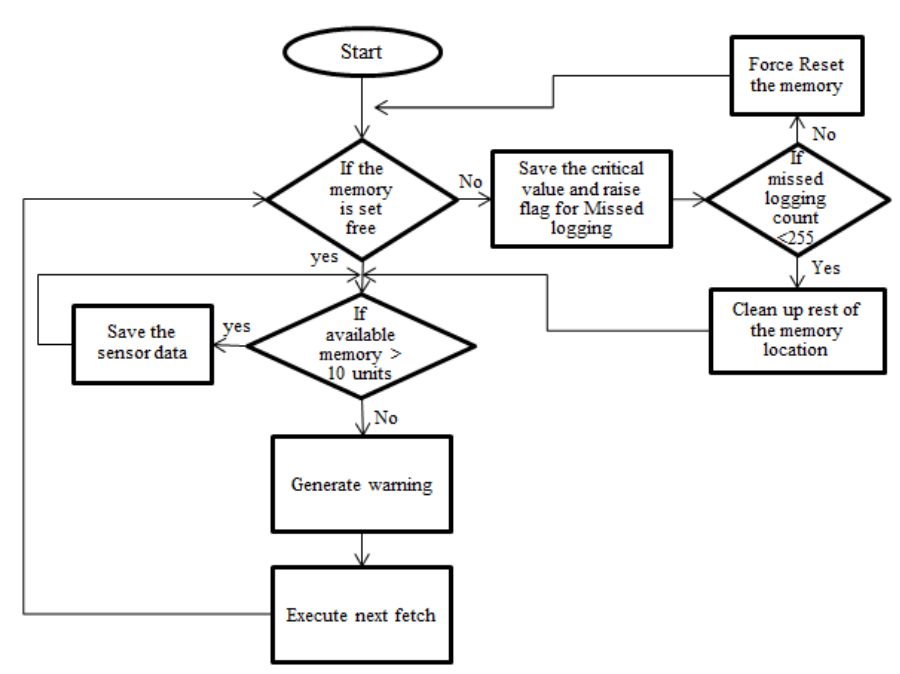

Figure 10: Memory management algorithm flowchart for the GSP-tag

transmitting the default EPC ID, the tag checks the reserved configuration block to get the proper user configuration. Unless any configuration is set differently, the tag starts running as a continuous transmission platform as it is set in the default configuration. But it can be changed anytime when the proper command is issued by the reader.

The commands $0 x X 000$ and $0 x X F F F$, written at the $1^{\text {st }}$ memory location, make the tag to run as Continuous data transmitting and Data logging Platform modes, respectively. The 
1st three hex locations, as shown in Fig. 8, switch the tag between two platforms. The data at the $4^{\text {th }}$ hex location configure the resolution of ADCs: $0 x 0 X X X, 0 x 1 X X X$, and $0 \times 2 X X X$ select 8-bit, 10-bit and 12-bit modes, respectively.

In the data logging mode, the main function of the GSP-tag is to periodically fetch data from the temporary buffer of the sensor thread and puts them in the user memory block during a certain interval set by the user. The command $G 2$ Write $=3,1,0001-F F F F$ written in the $2^{\text {nd }}$ reserved memory location sets any integer interval from $1 s$ to $65535 s$. This feature enables the users to choose their own preferred frequency of data acquisition depending on the size of the available user memory and the total uninterrupted time

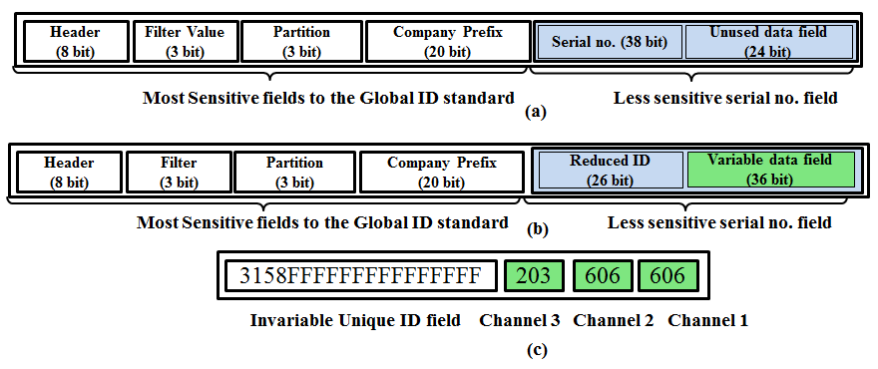

Figure 11: (a) Schematic of the SSCC-96 EPC ID standard (b) Modified version of the SSCC-96 EPC ID Standard. (c) A schematic of the prototyped GSP-tag's transmitted ID.

allowed between two consecutive data transmission periods. The arrangement is to ensure that the space in the user memory block is not used up. In some applications, the frequency of data acquisition is fixed and should strictly be maintained. With the new feature for this tag, the users can schedule their own data transferring periods towards their preferences. With this configuration the user memory is always checked for the space availability for the next fetch to accommodate. To the MPU, whenever the memory block appears to be almost full, it would notify the server/reader through the modified EPC ID transmission 
with the special notations at the sensor data field. Table I lists all the configuration commands.

\subsubsection{Memory Management}

In a typical Gen2 tag, the size of the available user memory is rather limited. For example, Monza 4 tag has usable memory size of 512 bits [65]. Depending on the available resources of the microcontroller, there may be a larger memory space available. But it still may not be adequate to accommodate all the data acquired, unless an effective mechanism is designed and applied in this scenario.

In the continuous- transmission mode, it is unnecessary for the GSP-tag to configure or manage the user memory block, since the data are continuously transmitted to the reader or the remote server. So the data storage is not needed. But in data logging mode, an effective memory management scheme is necessary for storing the data locally.

In our design, we have reserved $512 \mathrm{Kbit}$ of memory space as the user memory. The whole memory block consists of units with a size of 16 bits and they are initialized with a value of 0xFFFF. As we discussed, any valid sensor data value must be within 0x0000 to 0x09C4 range. Hence, an initialized 0xFFFF value at any memory location indicates to the MPU and/or a high level application developer that this memory is available for filling in new data. Within any memory unit, 1st 3-hex locations are used to store the sensor data and the 4 th one is used as the identifying number of the channels $(\mathrm{CH}-1, \mathrm{CH}-2$ \& $\mathrm{CH}-3$ represented by $1,2 \& 3$, respectively). The data from the consecutive 3 -channels are saved in consecutive locations in the memory, as shown in Fig. 12. While in the data logging mode, 
TABLE III

Power Budget in Data Logging Mode

\begin{tabular}{lcccc}
\hline \hline \multicolumn{1}{c}{ State of the Platform } & $\begin{array}{c}\text { Time in state } \\
(\mathrm{ms})\end{array}$ & $\begin{array}{c}\text { Current } \\
(\mu \mathrm{A})\end{array}$ & $\begin{array}{c}\text { Current*Time } \\
(\mu \mathrm{A} * \mathrm{~ms})\end{array}$ & $\begin{array}{c}\text { Battery life } \\
(\text { years })\end{array}$ \\
\hline Sleep : & 998.432 & 0.9 & 898.59 \\
$\begin{array}{l}\text { MPU in stop with RTC } \\
\text { state }\end{array}$ & & & \\
Sensor off & & & \\
Data acquisition: & 1.56 & 219 & 341.64 \\
$\begin{array}{l}\text { MPU in low power run } \\
\text { state; }\end{array}$ & & & \\
Sensor on & & & \\
Total in each second \\
period
\end{tabular}

the MPU manages the memory based on an internal data management algorithm, with its flowchart shown in Fig. 10.

The MPU keeps track of the availability of the space in the User memory for the next data fetch. If the memory appears to be filled out, it would notify the server/reader about the condition through the modified ID transmission. Any standard Gen2 read command (i.e. ReadG2 31 0000-FFFF) can be used to retrieve the data from the memory. After reading the data, a 0x0000 command (i.e. G2Write $=3,2,00$ 00) is written to the 3rd configuration block resetting each of the memory units, other than the $1^{\text {st }}$ and the $2^{\text {nd }}$ configuration blocks, 
TABLE IV

Power Budget in Continuous Data Transmitting Mode

\begin{tabular}{llll}
\hline \hline \multicolumn{1}{c}{ State of the Platform } & Time in state $(\mathrm{ms})$ & $\begin{array}{c}\text { Current } \\
(\mu \mathrm{A})\end{array}$ & $\begin{array}{c}\text { Battery life } \\
(\text { days })\end{array}$ \\
\hline $\begin{array}{l}\text { Continuous sensing and } \\
\text { streaming MPU on }\end{array}$ & Always & 36422 & 15.57 \\
Sensor on & & & \\
\end{tabular}

to the value of $0 x F F F F$ and making them available for the next session. If data are not transferred before the user memory runs out of space, this data logging is considered as a Missed data logging forcing the MPU to keep the very basic information of this session. In this scenario, the MPU performs internal data squeezing operation—saves the minimum, maximum and the average values of the whole data collected along with the channels identifying number - and frees the rest of the memory for the next session of data logging. In the meantime, the MPU modifies its EPC ID accordingly to keep track of this incident and to let the server/reader be aware of this incident through transmitting the modified ID, i.e. 3158 FFFFFFFFFFA01A01A0, showing the 1st instance of missed data logging, when the tag comes within the next available reader's interrogation zone. If the same type of incident occurs again before any kind of memory reset takes place, it keeps increasing the number stamp of the counting reference, i.e. 0xA02A02A02, and modifies the ID accordingly. This design limits the maximum number of missed data logging incidents that the GSP can track to $255(0 x X F F)$ before it forcefully resets itself. All the previous data are lost as a result. 


\subsubsection{Modified EPC ID Generation and Sensor Data Communication}

We adopted the SSCC-96 (Serial Shipping Container Code) EPC standard to accommodate the data from multiple channels along with the GSP's identification mechanism [70], [71]. Since no Global standard for RFID sensing applications ever existed, the EPC Global tag data standard has been used to generate the ID for the GSP-tag. However, while designing the scheme for the ID modification, we paid a careful attention to maintaining the integrity of the EPC Global Tag Data standards, so that it does not conflict with any other global standards or lose its unique identity in a broader sense. Therefore, the existing standard ID systems have been analyzed very closely to identify the different parts of the ID sequence and their functions.

A typical EPC ID consists of Header, Filter, Partition, Company prefix and Serial No. fields. Out of these basic fields, the Header, Filter, Partition and the Company prefix fields are directly related to the EPC ID standard formation and the primary global identity of the product, whereas the Serial No. represents the secondary identity, i.e., representing different products within a certain company. Hence, the relatively less significant Serial No. field has become our ultimate choice as a carrier of variable sensor data. In the prototype design we have implemented the SSCC-96 EPC data standard to generate the ID for the GSP tags. In the modified ID, the Serial No. field is divided into two new fields: Reduced unique ID field and Variable data field. Reduced ID gives the tag a unique identity among the products in certain company level and the Variable data field carries the sensed information. The variable data field is further sub-divided to carry information from all 
three channels. Fig. 11 shows the standard SSCC-96 ID fields and the modified version in our design.

In the continuous transmission mode of operation, the data acquired from the sensors are sent as millivolt and represented as a corresponding hexadecimal value in the ID. To accommodate all possible values with up to about 2500 different levels depending on the maximum resolution of the ADC, 3-hex locations have been allocated for each of the channels.

Thus, all available 9-hex-locations in Fig. 11c are used for three channels and all of them are identical, which makes it very difficult for the application software or backend server to distinguish the data coming from different channels. This issue is solved in our design by clearly identifying the physical locations of the channels in the tag in Fig. 6 and their corresponding data locations in the ID in Fig. 11c \& Fig. 14 or inside the user memory in Fig. 15 as well as strictly stipulating the MPU or the application software to access that particular locations for the data from the corresponding channels.

In the data logging mode, the data is not directly sent over the ID; instead they are accessed using standard read command. The ID data channel is just used to send the primary information and the possible warnings about the usage of the memory blocks to the backend server. For example, a command of 3158FFFFFFFFFFA00A00A00 notifies the server that the tag is running in data logging mode and the memory is not yet filled. But once the memory appears to be filled up, the GSP-tag keeps transmitting 3158FFFFFFFFFF FFFFFFFFF to warn the server about this critical situation until it runs out of space in the user memory. However, if the data is not transferred and the memory is 
not reset, the GSP will perform internal data squeezing and modify its ID to transmit 3158FFFFFFFFFFA01A01A01 notifying the server that the 1st instance of internal data squeezing has taken place inside the memory. If this same situation repeats before the memory reset is performed, the GSP will keep increasing the count number after each instance takes place and may go all the way up to the maximum count number 0xXFF before it forces itself to reset. Table II summaries the sensor data structure of the GSP-tag.

\subsubsection{Power Budget and Battery Lifetime Estimation}

The GSP is powered by a 3.0V MnO2(Li+) coin battery (CR2354) with the current of about 560mAh [73]. Being a semi-passive platform, the power consumption of the GSP is generally determined by the total current used by the MPU and the appended sensors.

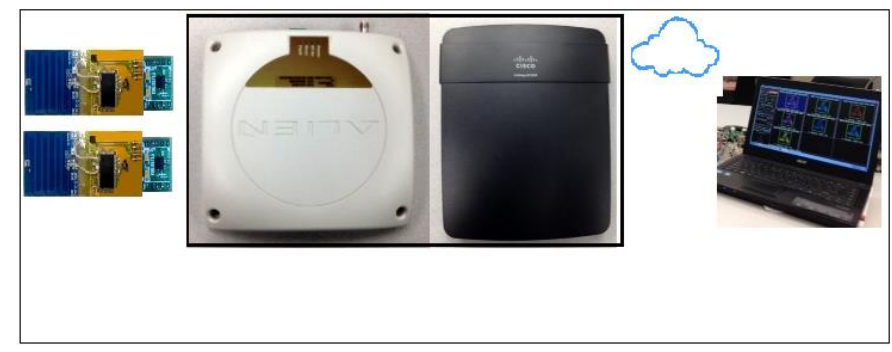

Figure 12: The GSP, deployed in a 3-tier IoT system monitoring a package in a smart supply chain monitoring scenario.

Considering the different requirements in different modes of operation for the platform (Data Logging mode/Continuous Transmitting mode), the MPU is programmed to switch between the low power states (Low Power Run state, Stop with RTC state) and the Normal Run state [74]. It is observed that in a certain mode of operation, the power consumption of the platform is dominated by some MPU's operational states that take the majority of its operation time. 
In the Data Logging mode, MPU is put into the Stop with RTC mode with a current of $0.9 \mu \mathrm{A}$ between two fetches, and is periodically wakened up to the Low Power Run state $(9 \mu \mathrm{A})$ to acquire data. It keeps doing so until it is interrogated by a reader. Once it is interrogated by the reader, the MPU switches to the Normal Run state (3412uA@214 $\mu \mathrm{A} / \mathrm{MHz})$ and after finishing the communication it immediately returns back to the low power state. Thus in this mode, it spends most of the time in Stop state thereby making the Stop state's current the dominant factor in the power consumption.

To calculate the minimum lifetime in Data Logging mode, we need to consider the maximum achievable data acquisition frequency ( 1 sample/s). With this configuration, it takes about 3 hours for the tag to fill out the whole internal memory $(512 \mathrm{~Kb}$ or $64 \mathrm{~KB})$. Although once in every $3 \mathrm{hr}$ is good for running the RFID chip, considering the wide variety of users' requirements, we are making an educated assumption that the RFID chip runs in every 30 minutes. With this setup and the assumptions, our calculations show that this platform can operate for at least 36 years without the need of changing the battery. For the running time estimation, battery and the MPU's leakage current losses were disregarded. In addition, it is assumed that within average 30 minute periods the platform is not inquired by any reader. Even if it is inquired, in some random cases, the MPU is obliged to go back to the Normal operational state after completing the operations. However, it is expected that this kind of random actions will not make any significant impact on the expected battery life time. Table III lists the parameters used in power budget calculation. 
TABLE V

Comparison Table of Few State-Of-The-Art Tags

\begin{tabular}{|c|c|c|c|}
\hline RFID Platform & Available features & $\begin{array}{l}\text { Read } \\
\text { range }(\mathrm{m})\end{array}$ & Average current consumption \\
\hline $\begin{array}{l}\text { RFID (2-chip) } \\
\text { platform[60] }\end{array}$ & $\begin{array}{l}\text { Battery assisted data logger } \\
\text { Non re-configurable }\end{array}$ & 3.4 & $0.7 \mu \mathrm{A}$ \\
\hline WISP-PDL [59] & $\begin{array}{l}\text { Wirelessly re-chargeable } \\
\text { capacitor assisted data logger } \\
\text { Non re-configurable }\end{array}$ & 4.5 & $500 \mu \mathrm{A}$ (Active) $1.8 \mu \mathrm{A}$ (sleep) \\
\hline $\begin{array}{l}\text { SenTag pool safety } \\
\text { monitor [77] }\end{array}$ & $\begin{array}{l}\text { Active, 1-bit drowning } \\
\text { monitor with personally } \\
\text { adjustable settings. }\end{array}$ & $\begin{array}{l}\text { Not } \\
\text { reported }\end{array}$ & $<150 \mu \mathrm{A}$ (approximately) \\
\hline $\begin{array}{l}\text { GSP-tag } \\
\text { (This work) }\end{array}$ & $\begin{array}{l}\text { Battery assisted, dynamically } \\
\text { reconfigurable RFID sensing } \\
\text { platform }\end{array}$ & 5.6 & $\begin{array}{l}1.743 \mu \mathrm{A} \text { (data logging mode) } \\
3622 \mu \mathrm{A} \text { (continuous data } \\
\text { transmitting mode) }\end{array}$ \\
\hline
\end{tabular}

In the Continuous Data Transmitting mode, the MPU and the sensors are required to run all the time. In this mode, MPU's Normal Run state current $(3412 \mu \mathrm{A} @ 214 \mu \mathrm{A} / \mathrm{MHz})$ and the sensor current $(200 \mu \mathrm{A}$ for the acceleration sensor [75] and $10 \mu \mathrm{A}$ for the temperature sensor [76]) become the only factors in battery lifetime calculation. The calculation shows that in this mode the CR2354 battery can last for 15.57 days. The detailed results are summarized in Table IV. Our analysis shows that this new platform exhibits a comparable current consumption level with other sensor tags while offering higher functional complexities with a respectable operational range of 5.6 meters. Table $\mathrm{V}$ shows performance comparisons of some contemporary tags in terms of the current consumptions, operational ranges and functional complexity.

\subsection{Experimental IoT System}

To test the GSP-tag's ability of working as a generic sensing platform in the existing IoT system, we have implemented an experimental 3-tier IoT system, as shown in Fig. 4, in the 
laboratory. Fig. 12 shows the constituting equipment in all three different tiers of the IoT system. With this setup, the GSP is equipped with a temperature sensor and a 3-D tilts angle sensor to monitor the ambient temperature and the tilt angle of a general supply package. In the system the GSP works as a tier-1 component—sensor node/platform.

It acquires information from the object that the tag is attached to and sends it to the routing tier. In routing tier (tire-2), a RFID reader (ALR-9650) maintains the primary communication with the GSP over the RFID protocol stack (EPC Gen2 standard) and sends the data to the internet gateway over the Ethernet protocol stack (IEEE 802.3). A CISCO

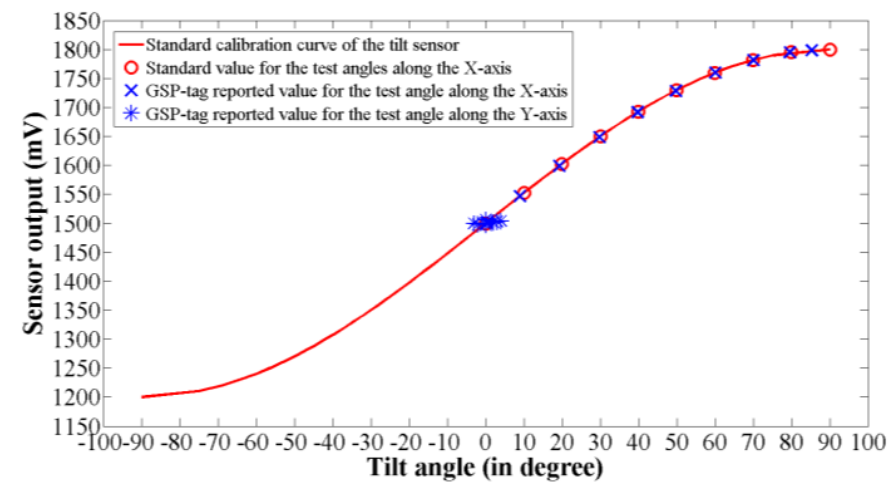

Figure 13: Plot of the tilt sensor data reported by the GSP-tag.

router (Linksys E1200) is used as the internet gateway. The data is then routed to the cloud/server (tier-3) (the IP address for the gateway to access the lab server is http://131.94.112.173). Controlling the GSP tag, monitoring and data analysis were performed from a remote server station through this gateway.

\subsubsection{Testing Results}

With the introduced experimental IoT system, we performed two different tests. In the 1st test the GSP-tag ran in the Continuous data transmitting mode and in the 2nd one it ran in 
the Data logging mode. A3-D tilt sensor and a temperature sensor were used in the tests to acquire the data.

$$
\theta_{x, y, z}=\sin ^{-1}\left(\left(V_{T \mid x, y, z}-1.5\right) \times \frac{1}{\text { Sensitivity }}\right)
$$

The ADXL330 is a 3-D (Z, Y \& Z) tilt sensor we used in the system. It generates a voltage response at the certain output pins corresponding to the inclination in that direction with respect to its horizontal position. According to the manufacturer data sheet [75], the sensitivity of the sensor is $300 \mathrm{mV} / \mathrm{g}$ in any direction and the rest voltage is $1.5 \mathrm{v}$ when it

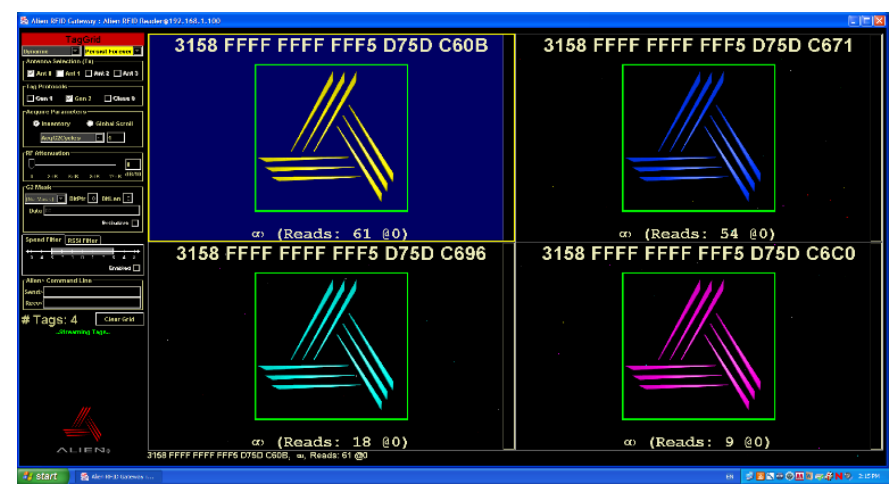

Figure 14: Picture shows the snap shot of the Alien reader's software interface showing

lies exactly parallel to the horizon. Eqn. 2 gives the relation between the tilt angle and the generated voltage where $\theta$ is the angle and $V_{T}$ represents the corresponding output voltage.

On the other hand, the output voltage response of the temperature sensor, MAX6605MXK+T, maintains a linear relationship to the ambience temperature with the sensitivity of $10 m V^{\rho} \mathrm{K}$, producing $0 m V$ for $0^{\circ} \mathrm{K}$ and $3730 \mathrm{mV}$ for $373^{\circ} \mathrm{K}$ [76]. To keep the temperature sensor's output voltage within the GSP's sensing range between 0 and $2500 \mathrm{mV}$, we placed a voltage divider between the sensor and the channel. This divider 
reduces the output voltage of the sensor by 2 and makes to vary from $0 m V$ to $185 m V$ for the full scale temperature change.

The reader interface software provided by the Alien was used to extract information sent by the tag. Since we did not design the application software, which is also beyond the scope of this study, we manually accessed the calibration curves of these sensors to interpret this measured data. Finally we compared the results with the standard values obtained from the same object using the classic tools.

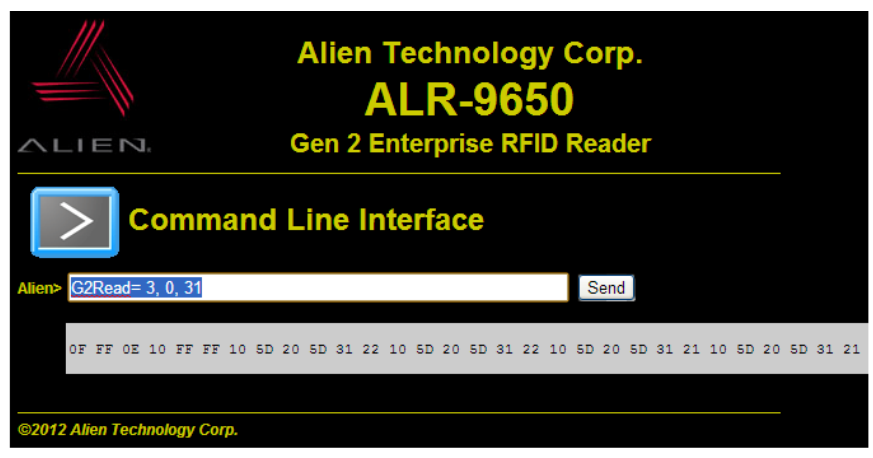

Figure 15: Picture shows the snap shot of a conventional G2 read command, reading the User

In the $1^{\text {st }}$ experimental setup, with the GSP-tag running in the continuous mode, the tilt angle of the horizontal plane was varied but the ambient temperature was kept fixed. In this setup the acceleration sensor was placed in such a way that, for an upright package, its $\mathrm{XY}$ plane lies parallel to the horizon and the Z-axis lies perpendicular to the horizon. Since we were interested in monitoring the tilt angle of the horizontal plane of the box, the $\mathrm{X}$ and Y axes were connected to the channel-1 and channel-2, respectively, and the $3^{\text {rd }}$ channel of the GSP was connected to the temperature sensor to monitor the ambience temperature of the box. With the GSP-tag running in the continuous transmitting mode, the box was tilted 
in different angles in the $\mathrm{XY}$ plane-basically along the $\mathrm{X}$-axis, a controlled variation of the inclination from $0^{0}$ to $90^{\circ}$ at an interval of $10^{\circ}$ was made, whereas the $\mathrm{Y}$-axis was kept fixed at $0^{\circ}$-and the last 6-hex location (1st 3-hex location for X-axis data and the next 3hex location for Y-axis data) of the transmitted ID of the tag was read to record the tilt data. Fig. 13 shows the snapshot of the ALR-9650 reader's interface capturing different IDs transmitted by the GSP-tag. The obtained results are then plotted on top of the standard calibration curve for comparison and performance analysis.

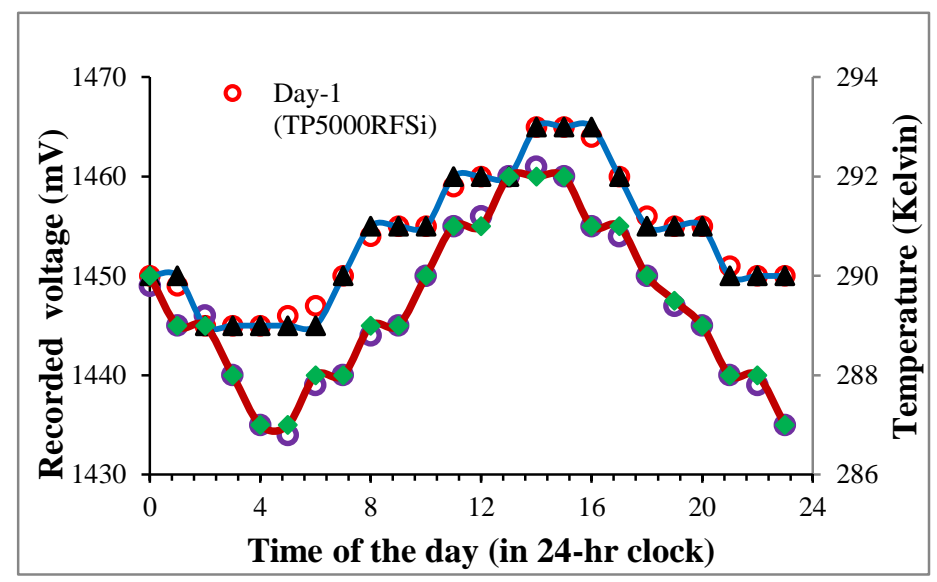

Figure 16: Picture shows the snap shot of a conventional G2 read command, reading the User

Fig. 14 shows the plot of the tilt sensor data. In this graph, it is found that, in $80 \%$ cases, the standard values and the GSP reported values for the test angles along the $\mathrm{X}$-axis coinsides. The similar results are observed for the Y-axis values, which localize around the $0^{\circ}$ angle $(1500 \mathrm{mV})$. The little deviation in the $\mathrm{Y}$-axis values is probably because of the hand jittering while changing the angles in X-axis direction. In Fig. 13, it is also observed that the $3^{\text {rd }}$ channel's location reads $0 x 5 D 7$ (equivalent to $1495 \mathrm{mV}$ ), all the time. The result shows that at the time of experimenting the room temperature did not vary and it was $299^{\circ} \mathrm{K}$. 
In the second experimental setup, where the GSP was running in the data logging mode, the package was put in a room without any RFID reader in the proximity. The package was left there for over the course of 48 hours with the data acquisition/fetching frequency of the tag set to be once per hour. Over this course of the test period, the tilt angle of the package was not varied. The only thing changed was the ambient temperature of the box. Finally the recorded data were extracted by the Alien Reader and plotted for the testing period. For the validation of the obtained data, they have been compared with another set of data obtained from TP5000RFSi used in the same experiment. Fig. 15 shows the snapshot of the data-log, extracted by the $A L R-9650$ reader and accessed through a remote server. Fig. 16 is the plot of temperature variations - obtained from both of the GSP and TP5000RFSi - for the package over the course of the testing period.

\subsection{Conclusions}

This paper has presented the design, implementation and testing results of a reconfigurable, semi-passive RFID sensing tag working as a Generic Sensing Platform (GSP) for the future IoT. While used in IoT framework, it can dynamically be reconfigured to run as a Continuous data transmitting platform (Online mode) or a Data logging platform (Offline mode). For data acquisition, the tag offers three generic sensing channels that are used to collect the real-time information from any kind of active sensors. The sensitivity of the channels is set to be $1 \mathrm{mv}$ and they can continuously acquire data at 2500 different levels with the values between $0-2.5 \mathrm{v}$ and report them to the backend server. Being a dynamically re-configurable sensing platform and offering flexible sensor modularity this GSP-tag possesses a tremendous advantages over the existing sensing tags as generic sensor nodes 
in the future IoT design and development. An experimental mini-IoT system mimicking a supply chain has been developed and tested in the laboratory environment to evaluate the GSP-tap performance and the data obtained from the test show a close correlation between the measured data and the actual values. In more than $80 \%$ testing cases the GSP reported the correct values while in the rest of the cases the mild deviations were observed within a tolerable range. 
CHAPTER III

DESIGN OF AN ENERGY HARVESTING MODULE 


\subsection{Introduction}

Energy sustainability without an embedded battery is one of the major desired features in the design of next generation portable and/or implantable devices, especially for those used in remote monitoring and tracking. Currently almost all those devices are battery-powered. They require periodic maintenance such as changing the batteries, which is cumbersome and costly; and even sometimes impractical under some scenarios (i.e. in case of bodily implanted devices, performing body surgery to replace the implant battery is difficult) [78]. Therefore, it is imperative to have alternative powering sources that could sustain the whole duration of operation of the implants without resorting to using batteries. One of the possible solutions is RF energy harvesting $[78,79]$. This technology utilizes the ambient electromagnetic waves, (i.e HF, UHF ISM band, GSM, WiFi radio, etc.) captured via a specially-designed antenna, and further rectified and stored in a charge condenser to power the operation of the circuitry. However, for energy harvesting, the amount of the energy captured by an antenna is highly dependent on the antenna properties, such as the gain, impedance bandwidth, radiation pattern, and etc. These inherent antenna electric properties are decided by the physical parameters of the antenna, such as size, shape and material properties including the conductivity and loss tangent of the conductor and dielectric property of the substrate [80]. Thereby the design of energy-harvesting antenna is critical for improving the performance of the next-generation biomedical implants. Many researches have investigated the issues of improving conversion energy of the harvester system. In [81], the authors introduced a Tx and Rx coil pair for inductive coupled wireless power transmission link for the biomedical implants. In this work the operating frequency 
was $200 \mathrm{MHz}$ and the Rx-Tx maximum separation at the optimal harvesting scenarios of $224 \mathrm{uW}$ was reported to be $12 \mathrm{~mm}$ in distance, which is insufficient for most medical implant applications. In a similar work reported by Sim et al. [82], tested the performance of different patch antenna in harvesting wireless energy from ambient souse like GSM band, where the physical dimension of the antenna is this work is specially focused for the powering of outdoor static sensor nodes in which the reported size of the harvester antenna sounds commensurate. However, for the portable devices application, where the physical size is one of the biggest design constraints, a much smaller in size yet efficient antenna is highly desirable.

In this work we have proposed a novel architecture of a folded dipole meandering antenna with a grounded parasitic loop for ambient energy harvesting at ISM UHF band (885$940 \mathrm{MHz}$, centered at $915 \mathrm{MHz}$ ) to power the operation of the future brain implants. Our work is mostly focused on minimizing the physical size of the energy-harvesting antenna while maintaining or even increasing the power conversion efficiency. In this work, a novel design of the antenna was performed and the simulation and field testing results for the design were carried out. The physical size of the antenna is obtained as $41.3 \mathrm{~mm} X 17.5 \mathrm{~mm}$. The conversion efficiency of the antenna along with an L-matching network and a 5-stage Cockcroft-Walton voltage multiplier is measured as $0.005 \%$ at a distance of $1.5 \mathrm{~m}$ from the transmitting source. With a source transmitting at $4 \mathrm{~W}$, the received power is considered to be sufficient to drive a Cortex M0 processor. 


\subsection{Antenna Design}

The proposed antenna is a compact folded dipole meandering antenna. It was designed and simulated on AXIEM simulator available on AWR Microwave office simulator, and implemented with a $1 \mathrm{Oz} \mathrm{Cu}$ FR4 board. In our design, the substrate thickness was chosen to be $1.6 \mathrm{~mm}$ with a relative permittivity of 4.47 and a loss tangent of 0.016 and the copper thickness is $0.035 \mathrm{~mm}$. Usually the antenna dimension, especially the length of a dipole
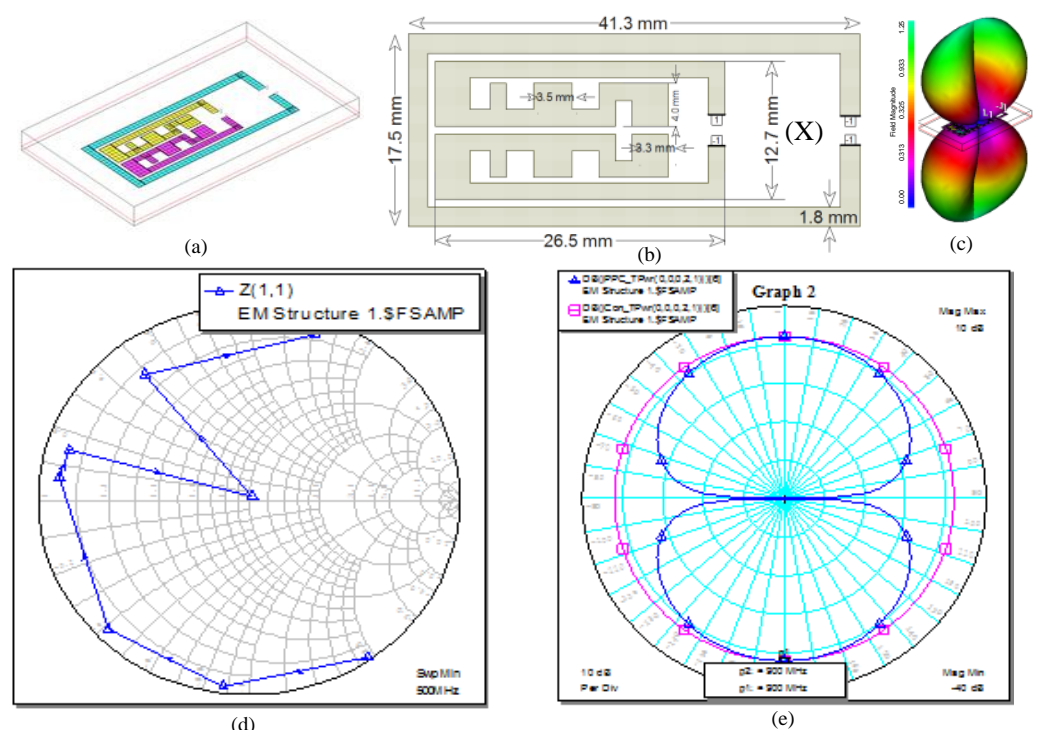

Figure 17: Implemented antenna layout and associated radiation pattern and input impedance plot on Stith chart.

antenna, decides the resonant frequency of the system. However, for a meandering antenna, the resonant frequency is mostly determined by the antenna geometry rather than the length itself. As proposed in [83], for a given frequency, the optimum gain is achieved with the highest radiation resistance and smallest possible DC loss resistance of the meandered pattern, where the radiation resistance is proportional to the vertical length of the meandered pattern and the loss resistance is inversely proportional to the total length of the 
line. Hence the optimization of the gain becomes a tradeoff among the physical size, radiation resistance and the loss resistance of the antenna.

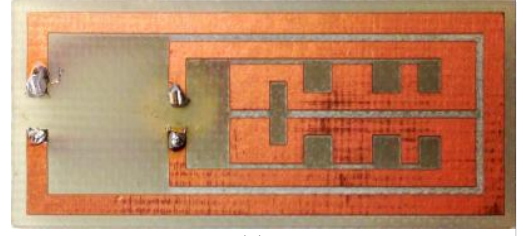

(a)

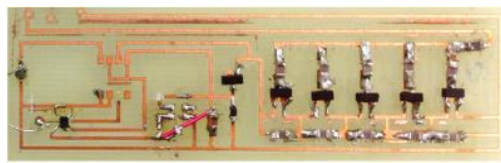

(c)

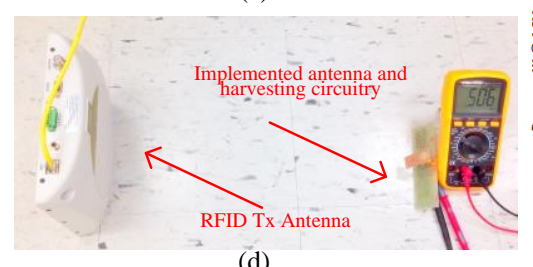

(d)

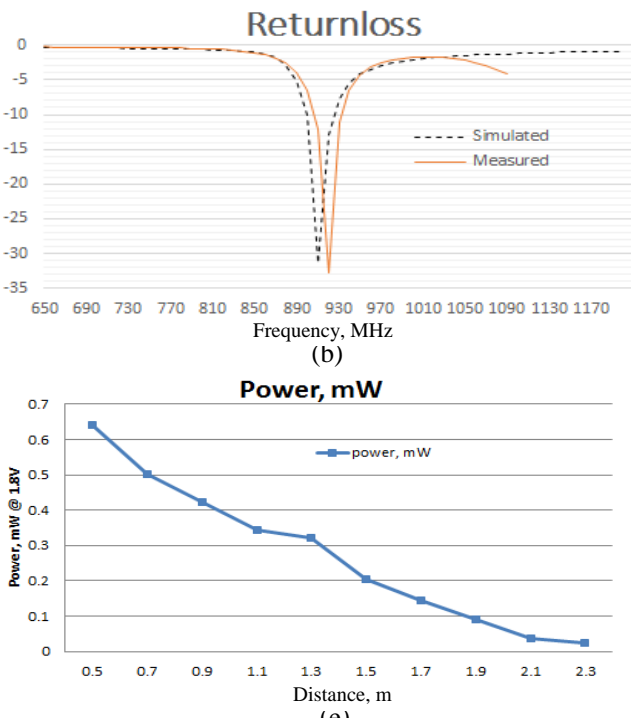

(e)

Figure 18: (a) Implemented antenna; (b) Simulated and measured return loss; (c) Implemented Cockcroft-Walton charge pump; (d) Snapshot of the test bench to measure harvested power; (e) Harvested power vs distance curve.

In our design, the thickness of the meandering line was optimized to take the loss resistance down to a given length of a conductive line. A relatively thicker line $(\mathrm{w}=1.8 \mathrm{~mm})$ was used for the layout of the meandering pattern. Fig. 17(a, b) shows the layout and Fig. 17(c, d \& e) shows the radiation pattern and input impedance of the designed antenna respectively. The design is consisted of two different sections including the active meandering dipole section, and the parasitic ground loop. The antenna fed point is connected to the active dipole section, whereas the parasitic ground loop operates as a resonant cavity (region $\mathrm{x}$ in Fig $1 b)$, where the cavity width is chosen close to $\lambda x / 2$, where $\lambda x=\lambda o / \sqrt{ }(\varepsilon r)$, and $\lambda o$ is the wavelength in free space. The length and width of the active patch is measured to be 
$20.5 \mathrm{~mm} \times 12.7 \mathrm{~mm}$ and with the ground loop included the total dimension of the antenna layout is measured to be $41 \mathrm{~mm}$ X $17.5 \mathrm{~mm}$. Fig 18 (a) shows the implemented antenna layout.

\subsection{Energy Harvesting Module Design}

The energy harvesting module is basically a 5 -stage charge pump that was designed following the Cockcroft-Walton voltage multiplier principle [84]. The circuit was implemented on a FR4 board with lumped components. In the design HSMS 285x and 286x Schottly barrier diode was used for its very low signal compatibility feature (Pin<$20 \mathrm{~dB}$ ), and very low leakage current loss. A $3 \mathrm{~V}$ Zener diode is used to make it a ripple free DC voltage at $3 \mathrm{~V}$ and finally the output is measured across a $1.8 \mathrm{~V}$ LDO. The input impedance of the harvester trace on FR4 along with all the component is measured 68$300 \mathrm{i}$.

\subsection{Testing Results}

The implemented antenna was measured for a variety of parameters such as the return loss, input impedance and bandwidth. Fig 18(b) shows the comparative study of the simulated and measured return loss (S11). The return loss was measured $33.78 \mathrm{~dB}$ at $917 \mathrm{MHz}$ and the simulated value was -31.8 at $910 \mathrm{MHz}$. The bandwidth was measured about $45 \mathrm{MHz}$ and the input impedance was measured as $48.58 \Omega$. Fig $18(\mathrm{c} \& \mathrm{~d})$ shows the implemented energy harvesting system in a test bench. The test bench was set up with a $915 \mathrm{MHz}$ RFID transmitting antenna (ALR 9650) as the radiating source with a power of $4 \mathrm{~W}$. The output voltage and the current at the harvester's output end was measured as a function of distance. 
Fig. 18 (e) shows the graphical representation of the testing results at various distances. At the optimal distance of $1.5 \mathrm{~m}$ the energy conversion efficiency is measured $0.005 \%$.

\subsection{Conclusions}

This article presents the design, implementation and measurement results of a $915 \mathrm{MHz}$ folded dipole meandering antenna for RF energy harvesting applicable for portable/implantable devices. Along with standard antenna measurements, a laboratory test bench was also setup to verify the performance of the antenna and the capacity of the associated energy harvesting circuitry. The implemented system was able to harvest around $200 \mu \mathrm{W}$ at an optimal distance of $1.5 \mathrm{~m}$ with the peak value of $647 \mu \mathrm{W}$ at a distance of $0.5 \mathrm{~m}$ from the radiation source. 
CHAPTER IV

DESIGN AND DEVELOPMENT OF SMART DEEP BRAIN STIMULATOR (SDBS) 


\subsection{Introduction}

Deep Brain Stimulation (DBS) is a surgical procedure used to stimulate specific brain regions with electrical signals to treat neurological disorders such as Parkinson's disease (PD) or dystonia $[85,86]$. With the procedure, the DBS electrode is implanted inside deep brain to deliver the electrical pulses to certain brain locations, most commonly the STN and/or GPi regions of the basal ganglia circuitry as a jammer to suppress the excessive neural firing to alleviate the PD's symptoms [87]. Despite being in use for PD treatment for a long time, current DBS devices remain to be technically desired in many aspects [8890].

One of the glaring deficiencies of the current DBS devices is their lacking of "smartness" in the operations. Firstly, current DBS electrode cannot synthesize and generate arbitrary stimulating waveforms based on analysis of the recorded brain data or the sensed patient's information. As a result, it is difficult to implement adaptive closed-loop DBS functions such as automatic waveform optimization and selection for best stimulation effect [90]. Secondly, current DBS devices are not considered to be "smart" because they are not connected to the Internet. Without online connection, DBS devices cannot take advantage of the powerful "cloud" computing resources of remote servers or cannot be monitored and/or controlled in real time by a clinical doctor or a DBS specialist from a remote location $[91,92]$.

Another major issue with the existing DBS systems is that they has not yet been able to take the advantage of powerful distributive computation - in to date reported DBS systems, a single processing core (implanted pulse generator-IPG) controls and monitors the activity 
of multiple electrodes [93-96]. A number of channels/electrodes being controlled by a single processor, this system requires relatively resource consuming, complex hardware and algorithmic processes to maintain coordination among the channels [97, 98]. Due to the sequential processing of multiple channels by a single controller, this architecture also has limitation on temporal resolution of real-time monitoring.

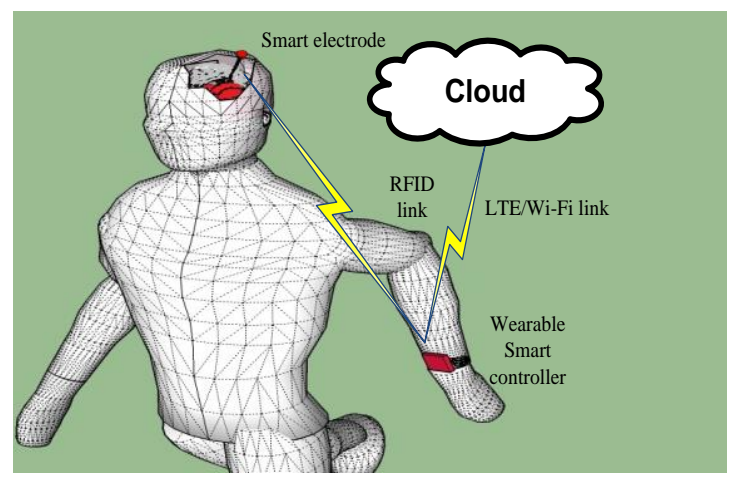

Figure 19: Illustration of the envisioned DBS system.

In addition, the communication backbone of the existing systems completely relies on the active communication technologies. This technology is subject to require a local oscillator, carrier multiplier, power amplifiers, complex modulation and demodulation circuitry for enabling high speed wireless communication, which consumes the much of the system power, and occupies the larger portion of the effective chip area. The use of this active technology is essentially hindering the process of miniaturization of the IPG, and making it power efficient enough to integrate it in each of the electrodes to enable discretization of the process. It is obvious that distribution of the work load implies requirement of the less resourceful hardware and software for the system, thereby enabling power efficient processing $[99,100]$. In addition, discretization and autonomy of the each of the electrodes 
will enable simultaneous operation - parallel and distributed processing of the brain activity at each of the micro brain sites-,thereby improving the temporal resolution of real-time monitoring, and offering faster response.

We envision that the next-generation ideal DBS device should be "smart" with powerful computing resources to evaluate the brain stimulation in real time. The new smart device should be internet-connected, and capable to stimulate and record data simultaneously with strong two-way communication capability. In addition, the new DBS electrode should be fully passive and/or wirelessly chargeable device with no or very minimal side effect.

In this work we propose a new smart DBS (SDBS) system with the above-described functions or capabilities. This proposed device can also be used as a general form of the future adaptive brain-sensing and modulation devices. This work details the architecture, design, prototyping, testing and evaluation of the proposed SDBS systems. The rest of the article is arranged as follows. The basic architecture of SDBS is introduced in Section II. The SDBS design and prototyping approaches are detailed in Section III. The testing and evaluation results are presented in Section IV. Finally, some conclusions are drawn in Section V.

\subsection{Smart DBS Architecture}

In this work, SDBS is designed and developed to contain a semi-passive (fully passive communication module and battery assisted processing core), secure, all-in-together, smart electrode that can run independently, and/or in coordination with an external controller. Figs. $19 \& 20$ show the conceptual structure of the smart electrode in the proposed SDBS 
system. According to the architecture, the electrode's internal processing core performs DBS operation locally. Hwever, for more advanced or computing intensive data processing and analysis, or in need of human intervention, the smart electrode can send the relevant data to the external controller through the established wireless communication link between it and the electrode (Fig. 21). To facilitate an efficient, bi-directional communication

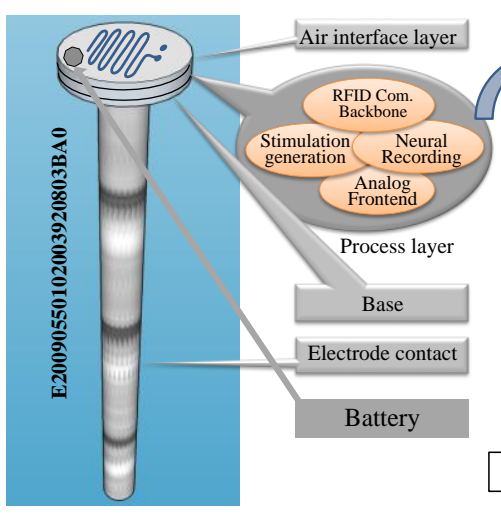

(a)

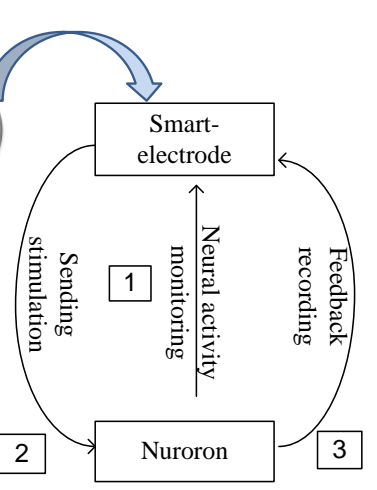

(b)

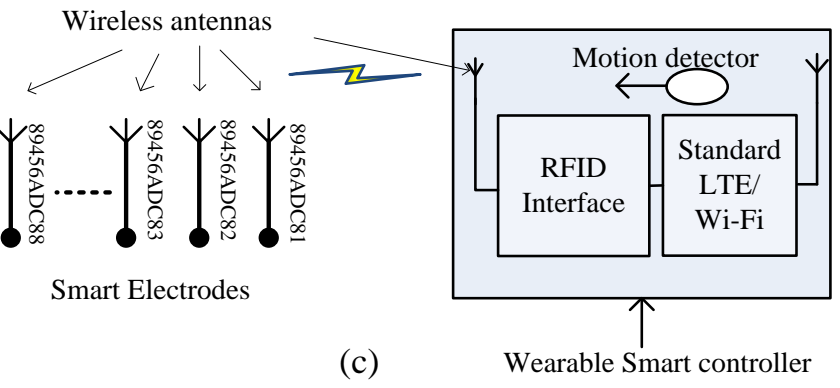

Figure 20: Basic structure of the Smart Deep Brain Stimulation (SDBS) system; (a) Schematic of a smart electrode; (b) Smart electrode's neural activity flow in the data communication layer: monitoring, stimulation generation \& feedback recording; (c) A multi-electrode SDBS system consisting of multiple smart electrode and the external controller where each of the electrodes has been assigned with a unique ID for the controller to target and to communicate with.

between the smart electrode and the backend controller, we have adopted the EPC Gen2 standard air interface protocol [69], which is widely used RFID communications, for data 


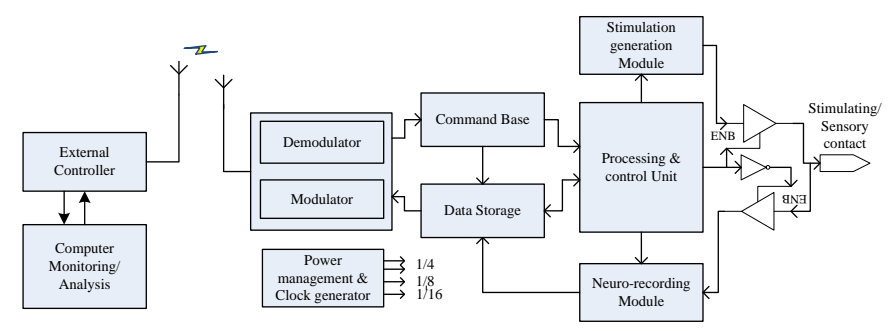

Figure 21: Schematic block diagram of the SDBS System.

communication between the smart electrode and the external controller. The standard EPC Gen-2 protocol flow has been carefully redefined to integrate and synchronize the DBS data processing and other computing operations within the system (Fig. 22). A new set of

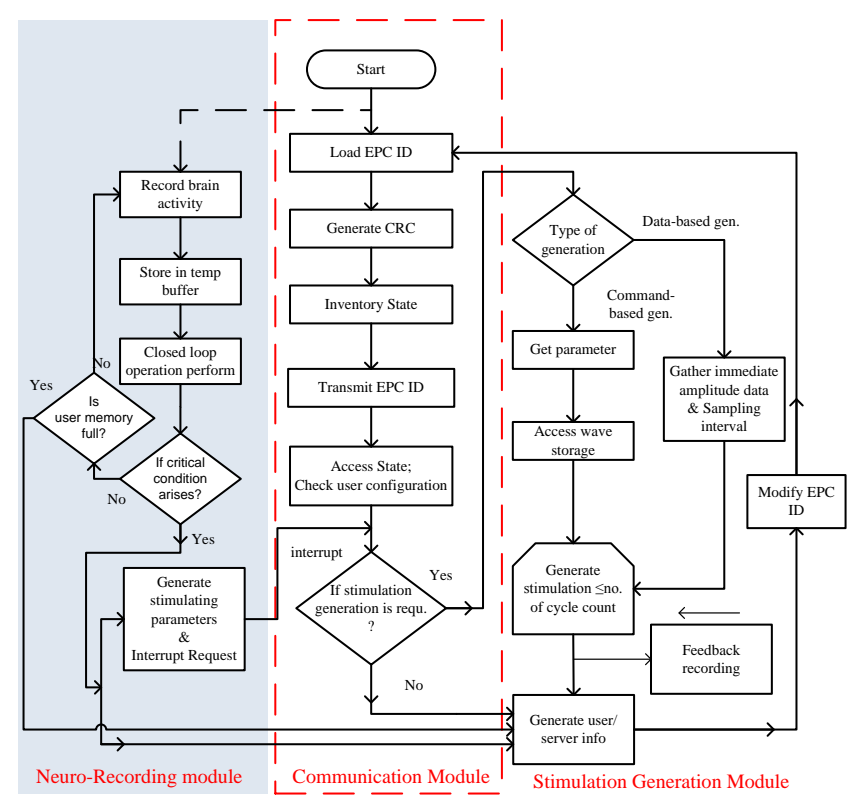

Figure 22: Optional flowchart of the electrode in the smart DBS.

commands has been defined to implement all the provable control and communication related to the DBS operation. Careful attention was paid to the protocol design process, so that the communication platform does not lose its integrity with the existing RFID standard in a broader sense. In addition, this has enables any regular RFID reader or mini reader, or wearable smart devices with the RFID protocol implemented to be qualified as the external 
controller. In the meantime, this controller can operate as the gateway to/from the remote computing-cloud via the LTE/Wi-Fi link.

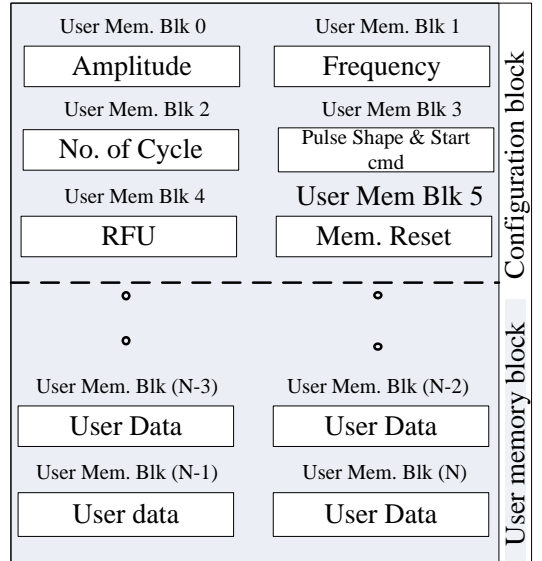

(a)

\begin{tabular}{c|c}
\hline $\begin{array}{c}\text { Bit } \\
\text { Value }\end{array}$ & $\begin{array}{c}\text { Memory } \\
\text { location }\end{array}$ \\
\hline \hline 00 & RFU \\
\hline 01 & EPC \\
\hline 10 & TID \\
\hline 11 & User \\
\hline \hline
\end{tabular}

(b)

Figure 23: a) Modified user memory structure of the reconfigurable SDBS platform; b) Memory space management in the conventional Gen2 RFID protocol.

\begin{tabular}{|c|c|c|c|c|}
\hline 11000111 & 11 & 00000001 & 00000100 & $\begin{array}{l}\text { 16-bit Data } \\
\text { blocks }\end{array}$ \\
\hline G2BlkWrite $\mathrm{cmd}$ & $\begin{array}{r}\text { Mem. target } \\
\text { Gen2 B }\end{array}$ & $\begin{array}{l}\text { Memory location } \\
\text { lock write parameters }\end{array}$ & Word count & $\begin{array}{l}\text { DBS configuration } \\
\text { parameter }\end{array}$ \\
\hline 11000011 & 11 & 00000001 & \multicolumn{2}{|c|}{ 16-bit Data block } \\
\hline $\begin{array}{r}\text { G2Write comd. } \\
\text { Gen? }\end{array}$ & $\begin{array}{l}\text { Mem. targe } \\
\text { Vrite param }\end{array}$ & $\begin{array}{l}\text { Memory location } \\
\text { ters }\end{array}$ & DBS config & ion parameter \\
\hline
\end{tabular}

Figure 24: Gen2 BlockWrite (data-based waveform generation command) and Write (commandbased waveform generation) commands.

The processing core of the electrode is designed to incorporate advanced DBS operational abilities. Along with recording neural activity, the processing module performs operation for symptom sensing and stimulation waveform generation. In addition to the conventional preset wave generation approach, this design has incorporated a novel data-based arbitrary waveform generation mechanism. With this new waveform generation technique, even the waveforms with an arbitrary shape that cannot be defined with a standard mathematical 
TABLE VI

Command Table for the Smart Electrode

\begin{tabular}{|c|c|c|c|}
\hline $\begin{array}{l}\text { Parameter } \\
\text { name }\end{array}$ & $\begin{array}{l}\text { Location in } \\
\text { the user } \\
\text { memory }\end{array}$ & Low level command format & $\begin{array}{l}\text { High level } \\
\text { command } \\
\text { format }\end{array}$ \\
\hline Amplitude & $\begin{array}{l}\text { Mem. blk } 0 \\
\text { (16-bit) }\end{array}$ & $\begin{array}{l}110000111100000000 \\
(0 \times A-0 \times 9 C 4)\end{array}$ & $\begin{array}{l}\text { G2Write }=3, \\
0,000 \mathrm{~A}- \\
09 \mathrm{C} 4\end{array}$ \\
\hline Frequency & $\begin{array}{l}\text { Mem. blk } 1 \\
\text { (16-bit) }\end{array}$ & $\begin{array}{l}11000011 \quad 1100000001 \\
(0 x A-0 x 9 C 4)\end{array}$ & $\begin{array}{l}\text { G2Write=3, } \\
1,000 \mathrm{~A}- \\
09 \mathrm{C} 4\end{array}$ \\
\hline No. of Cycle & $\begin{array}{l}\text { Mem. blk } 2 \\
\text { (16-bit) }\end{array}$ & $\begin{array}{l}110000111100000010 \\
(0 x 1-0 x F F F F)\end{array}$ & $\begin{array}{l}\text { G2Write=3, } \\
2,0001- \\
\text { FFFF }\end{array}$ \\
\hline $\begin{array}{l}\text { Wave Shape } \\
\& \text { Start cmd. }\end{array}$ & $\begin{array}{l}\text { Mem. blk } 3 \\
\left.\text { (1 } 1^{\text {st }} 8 \text {-bit }\right) \\
\text { Mem. blk } 3 \\
\left(2^{\text {nd }} 8 \text {-bit }\right)\end{array}$ & $\begin{array}{l}110000111100000011 \\
\text { (this position must be filled } \\
\text { from the value obtained } \\
\text { from Table II) }\end{array}$ & $\begin{array}{l}\text { G2Write }=3, \\
3 \text {, (Table II) }\end{array}$ \\
\hline Data Read & $\begin{array}{l}\text { Any mem. } \\
\text { location }\end{array}$ & $\begin{array}{l}1100001011 \text { (starting } \\
\text { mem. location, range) }\end{array}$ & $\begin{array}{l}\mathrm{G} 2 \mathrm{Read}=3, \\
\text { (mem range) }\end{array}$ \\
\hline
\end{tabular}

function can be generated for stimulation. The associated parameters of the waveforms such as amplitude, frequency and duration can be dynamically selected, optimized and adjusted. The incorporation of the arbitrary waveform generation into the existing waveform generation system has enriched the scope of adaptive stimulation generation, thereby could potentially improve the SDBS' performance significantly in the adaptive closed-loop neuro-sensing and modulation operation.

\subsection{Smart DBS Design and Prototyping}

The complete SDBS electrode design has two primary building blocks: an analog frontend, and a digital core. The analog frontend contains the antenna, matching network, modulation/demodulation circuitries and signal conditioning block. It is very similar to a 
TABLE VII

Associated Command Table for the Smart Electrode

\begin{tabular}{llll}
\hline \hline \multicolumn{1}{c}{ Wave shape } & \multicolumn{1}{c}{$1^{\text {st }} 8$-bit } & Last 8-bit & \multicolumn{1}{c}{ Command word } \\
\hline Sinusoidal & 0x01 & 0xAA & 0x01 AA \\
Mono phase square pulse & 0x02 & 0xAA & 0x02 AA \\
Bi-phase square pulse & 0x03 & 0xAA & 0x03 AA \\
Exponential pulse & 0x04 & 0xAA & 0x04 AA \\
Mono phase impulse & 0x05 & 0xAA & 0x05 AA \\
Bi-phase impulse & 0x06 & 0xAA & 0x06 AA \\
Negative ramp & 0x07 & 0xAA & 0x07 AA \\
Positive ramp & 0x08 & 0xAA & 0x08 AA \\
Gaussian pulse & 0x09 & 0xAA & 0x09 AA \\
Future use & 0x0A & 0xAA & 0x0A AA \\
-- & & -- & -- \\
Future use & 0xFE & 0xAA & 0xFE AA \\
Direct data-based generation & 0xFF & 0xAA & 0xFF AA \\
\hline \hline
\end{tabular}

conventional Gen2 RFID system with its structure detailed in [101]. The design of the digital core is of the prime interest in this section. It includes the crafting and implementation of the communication protocol standard neural activity recording \& processing module, stimulation waveform generation module and their intercommunication protocols. The waveform generation module is a sequentially accessible data generation system with the communication module. However the neuro recording \& processing module can access it at any time it is deemed to be necessary. On the other hand, the recording \& processing module, which includes sensing, local processing and temporary storing of the data, is completely parallel process with the rest of the processes in the microchip. Data security measures are also implemented in the 
TABLE VIII

Over the ID Communication Layout

\begin{tabular}{|c|c|c|c|c|}
\hline \multicolumn{5}{|c|}{$\begin{array}{c}\text { 96-bit ID system } \\
\text { E20090550102003920800000 } \\
\end{array}$} \\
\hline Unique ID & \multicolumn{4}{|c|}{ Variable data field } \\
\hline 84-bit & \multicolumn{4}{|c|}{ 12-bit } \\
\hline \multirow{3}{*}{$\begin{array}{l}\text { E20090550102003920800 } \\
\text { E20090550102003920801 } \\
\text { E20090550102003920802 }\end{array}$} & \multicolumn{2}{|c|}{$\begin{array}{l}\text { 8-bit missed data } \\
\text { logging warning }\end{array}$} & \multicolumn{2}{|c|}{$\begin{array}{c}\text { 4-bit critical } \\
\text { condition warning }\end{array}$} \\
\hline & 8-bit & Comment & 4-bit & Comment \\
\hline & $0 \times 00$ & $\begin{array}{c}\text { No missed } \\
\text { Data logging }\end{array}$ & $0 \times 0$ & $\begin{array}{c}\text { No } \\
\text { warning }\end{array}$ \\
\hline \multirow{3}{*}{ E20090550102003920808 } & $0 \times 01$ & $1^{\text {st }}$ instance & \multirow{3}{*}{$0 x F$} & \multirow{3}{*}{$\begin{array}{c}\text { Critical } \\
\text { condition } \\
\text { arose and } \\
\text { stimulatio } \\
\text { n applied }\end{array}$} \\
\hline & -- & -- & & \\
\hline & $0 x F F$ & $255^{\text {th }}$ instance & & \\
\hline
\end{tabular}

design. The digital core was implemented on an ARM CortexM0+ micro processing core available on ATSAMD20E18 micro-chip and has been further implemented on a custom designed PCB board. A combination of C and Assembly languages were employed for implementation of the digital cores and their intercommunications. The design will be detailed in the following subsections.

\subsubsection{Reconfigurable SDBS Platform}

As a suitable interactive platform for a client device, that runs in conjunction with the external device or controller, the inclusion of dynamic re-configurability - the ability to accept dynamic command to modify or alter the current state of operation of the associated systems - is one of the prime criterion. Existing RFID platform severely lacks in flexibility in this aspect. Normally they only allow the user to set/reset the passcode of the access; no dynamic commands can be issued through the RFID protocols. Furthermore, EPC protocol 
cannot carry/understand non-EPC standard data and commands. It also lacks in true bidirectionality in its communication - only the reader can initiate any communication. To facilitate a broad spectrum of re-configurability, and to make the system compatible for non-EPC standard communications, we have craftily modified the existing command

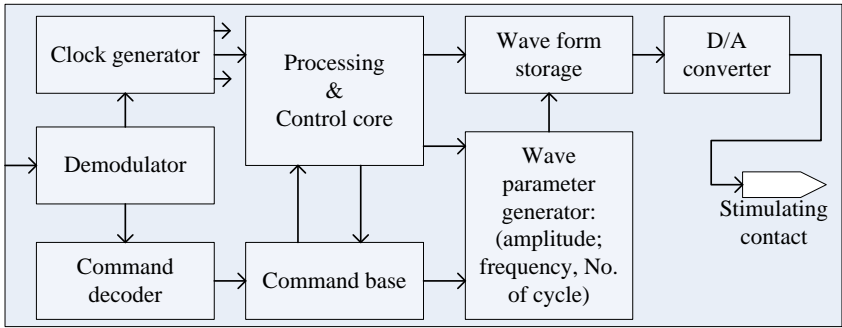

(a)

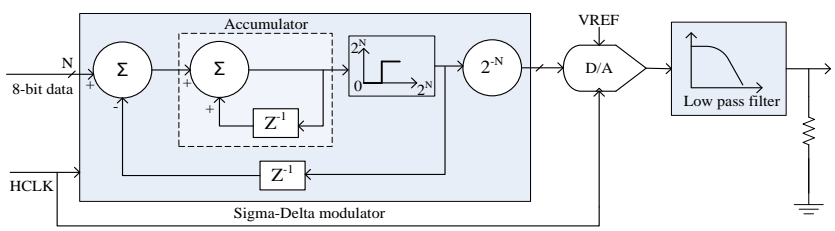

(b)

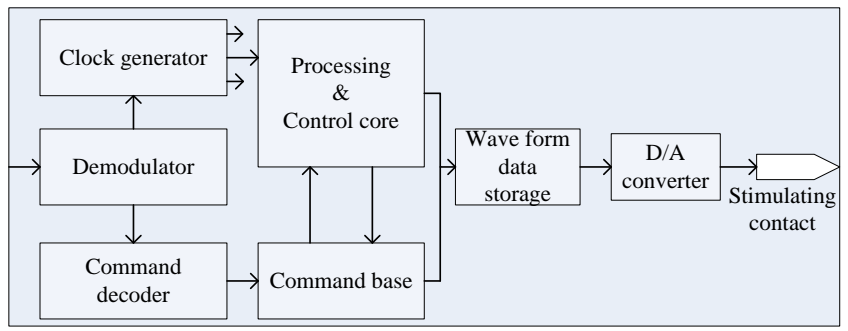

(c)

Figure 25: (a) Block diagram of the command-based stimulation generation module; (b) schematic diagram of the sigma-delta modulator as D/A converter; and (c) block diagram of the data-based waveform generation module.

execution flow of the current EPC Gen2 standard and introduced a novel set of commands to infuse re-configurability with it. The flow diagram in Fig. 22 shows the modified command execution flow in the introduced reconfigurable system. In the new system, part of the User memory section has been used to store the SDBS configuration command. User 
memory section is typically used to store user data, which in this design will be used as the storage for the recorded neural data. However, a part of the user memory has been declared as the configuration memory that is made unavailable to the electrode for data storage. Fig. 23 shows a schematic diagram of the modified User memory structure. As per the design, the first 6 memory blocks (each block=16-bits) have been reserved to store the configuration parameters, where the 1st 4-locations are used for the DBS operational control, and the rest are used for the general control of the platform. As shown in Fig. 23, memory block 0 is used to define the amplitude of the waveform; memory block 1 is used to set the frequency and memory block 2 is employed to determine the number of pulses in

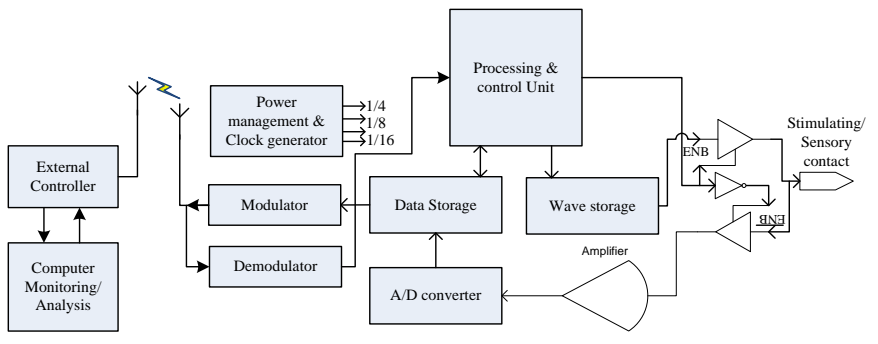

Figure 26: Schematic block diagram of the SDBS recording \& processing module

each stimulation session. The value could be set to any integer number from 0 to 65535 . In memory block 3 the first 8 -bits are used to choose the waveform type, and the last 8 bits store the command to initiate the stimulation process. Since it is designed in compliance with the RFID Gen2 protocol standard, the new system allows the standard G2Write command to modify the configuration parameters individually or G2BlockWrite command to modify them all together. Fig. 24 shows the formats of the two configuration commands. Table VI \&Table VII summarize all the commands and related parameters respectively that are used to enable non-EPC standard control and communications. The procedures described above are for the scenarios where the external controller initiates 
communications with the electrode, but not the other way around. To enable electrode initialized communication within SDBS, this design has used the Modified ECP ID transmission mechanism [101]. In this process, out of 96-bit ID, the first 84-bit has been used to provide the unique ID of the electrode to be targeted and addressed uniquely, and the rest of the digital bits have been used as the electrode generated warning carrier. Out
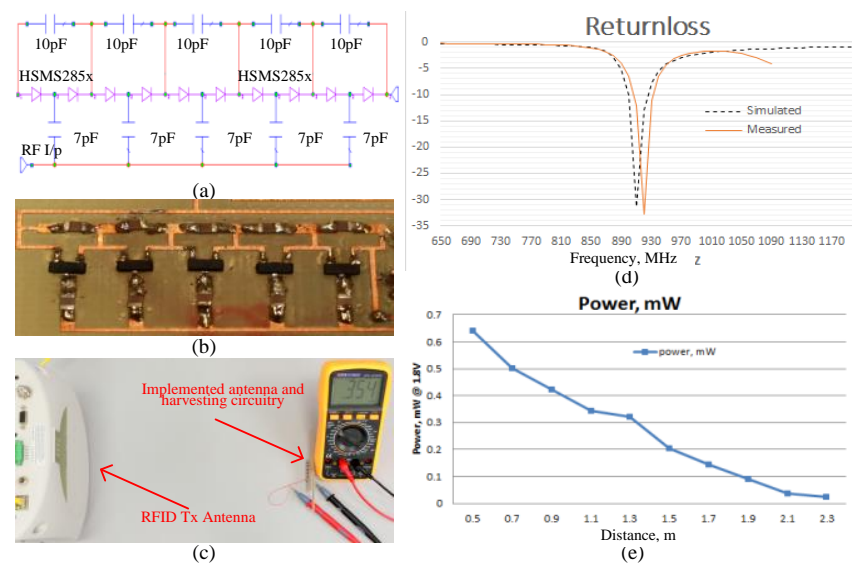

Figure 27: (a) Antenna impedance and radiation pattern; (b) Antenna return loss; (c) Implemented Cockcroft-Walton charge pump; (d) Snapshot of the test bench to measure harvested power; (e) Harvested power vs distance curve.

of the 12-bit data, electrode uses the very last Hex digit to send the warning to the controller about any critical situation detected, and the other 8-bit data were used to communicate on the memory information - whether the memory is full and needs to be transferred. This warning flags the controller in the backend to initiate communication with the electrodes. Table VIII summarizes the EPC ID data communication strategy adopted in the SDBS.

\subsubsection{Stimulating Waveform Generation Module}

In this work, we have implemented two different schemes to generate stimulation: (i) generating stimulation waveforms based on a command; and (ii) based on arbitrary 
sampled data from the brain. Fig. 25(a) illustrates the command-based waveform generation module in SDBS. A command decoder interprets the commands sent by the external controller and passes waveform parameters (i.e. amplitude, frequency, and stimulation duration) to the command base, where the command and its associated parameters are temporarily stored for the micro-processing unit. In the next few clock cycles, the processor accesses the waveform data storage to withdraw the waveform type and its mathematical function such as pulse, sinusoid, Gaussian, or exponential function.

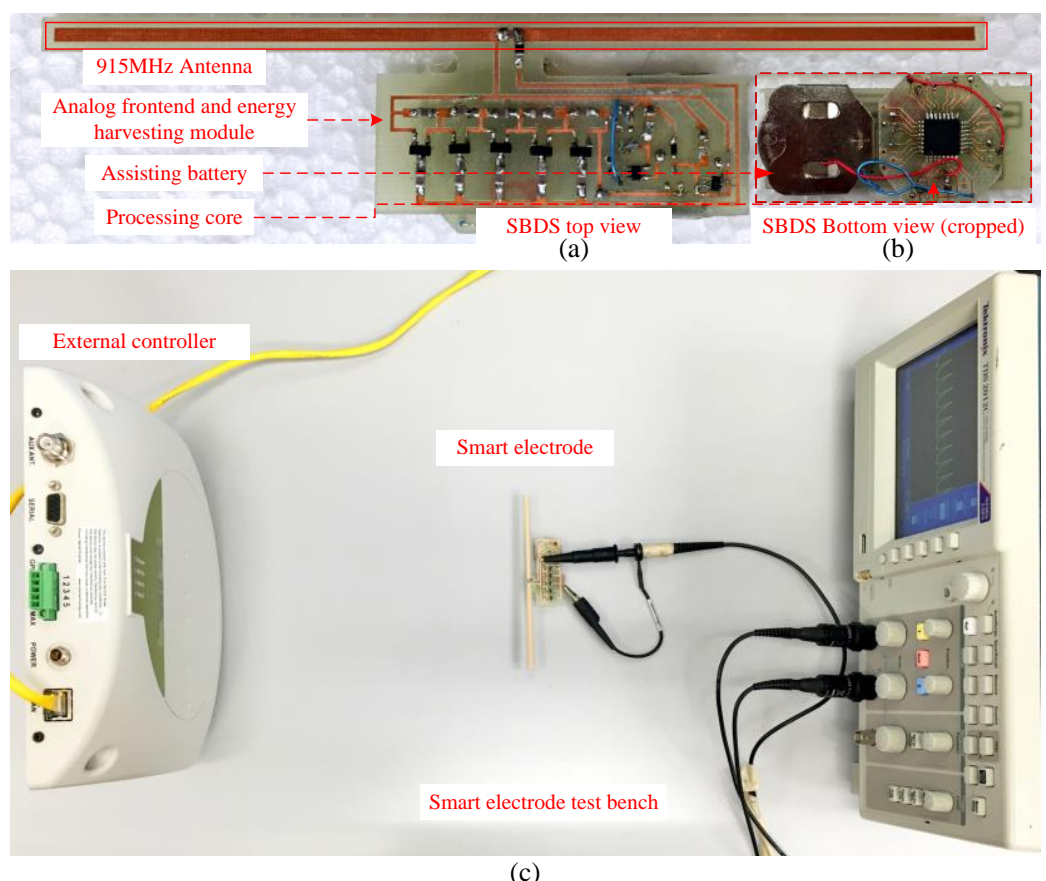

Figure 28: The SDBS system and its components; (a) the smart electro; (b) bottom view of the electrode; (c) SDBS test bench setup where an Alien RFID reader, ALR-9650, is used as the external controller.

A look-up table (LUT) is subsequently generated with the parameters stored in the parameter storage. The generated LUT contains a single period of the waveform consisting of 256 instantaneous amplitude samples of 8-bit each. The LUT data are then passed 


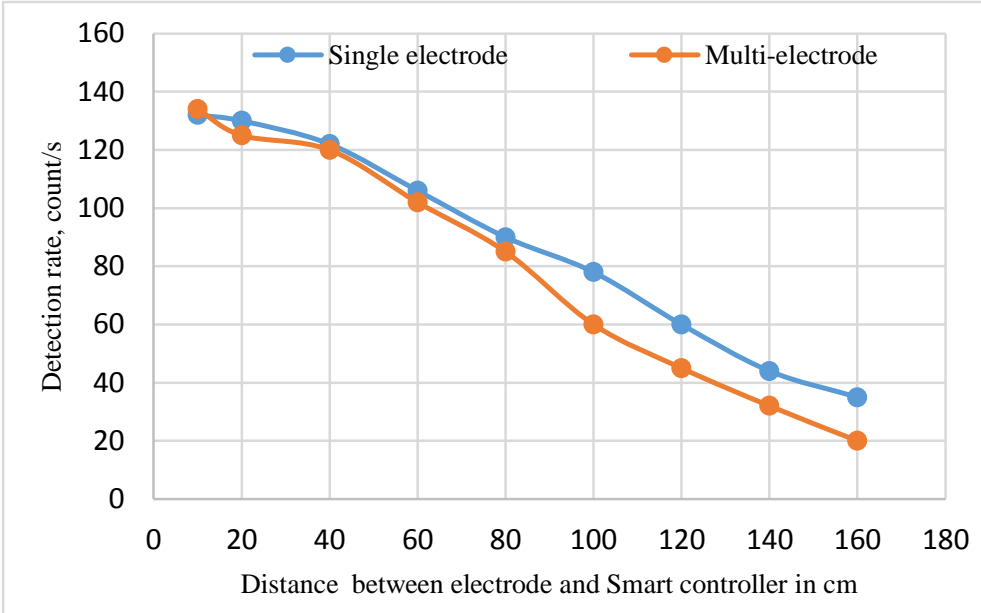

Figure 29: Maximum communication distance vs detection rate in single $\&$ multi-electrode scenarios. In the multi-electrode case, 5 extra static, electrodes were placed at the approximate $80 \mathrm{~cm}$ away.

TABLE IX

Comparative Study of Few Recent DBS Platforms

\begin{tabular}{ccc}
\hline \hline Platform & $\begin{array}{c}\text { Operating } \\
\text { mode }\end{array}$ & Power consumption \\
\hline Activa PC+S [93] & Fully active & Not reported \\
64 Channel & Fully active & DBS ASIC: $89 \mu \mathrm{W}$ (normal \\
Programmable Closed- & & operation mode) \\
Loop Neurostimulator [94] & & Config. Mode: $271 \mu \mathrm{W}$ \\
64-Channel UWB & Fully active & Comm. Module: $10.5 \mathrm{~mW}$ \\
Wireless Neural Vector & & DBS ASIC: $200 \mu \mathrm{W}$, \\
Analyzer SOC [95] & & Recording: $1.4 \mathrm{~mW}$ \\
& & Stimulation: $1.5 \mathrm{~mW}$ \\
SDBS [this work] & Semi-passive & Dignal processing: .26 mW \\
& & Comm. Module: $0.1 \mathrm{~mW}$ \\
& & (fully operational mode); \\
& & Low power mode: $1.2 \mathrm{~mW}$ \\
& & Comm. Module: passive \\
\hline \hline
\end{tabular}




\section{TABLE X}

Instantaneous Samples for Data-Based Arbitrary Waveform Generation

\begin{tabular}{ll}
\hline \hline Decimal value & $0,100,190,250,380,450,570,450,380,250,190,100,10$, \\
& $100,190,260,370,450,550,450,370,260,190,20,90,150$, \\
& $210,280,410,280,210,150,150,150,150$ \\
\hline Hex value & 0000, 0064, 00BE, 00FA, 017C, 01C2, 023A, 01C2, 017C, \\
& 00FA, 00BE, 0064, 000A, 0064, 00BE, 0104, 0172, 01C2, \\
& 0226, 01C2, 0172, 0104, 00BE,0014, 005A, 0096, 00D2, \\
& 0118,019A, 0118, 00D2, 0096, 0096, 0096, 0096 \\
\hline
\end{tabular}

and a low pass filter, as shown in Fig. 25(b). The LUT output is then repeated through the D/A converter to generate a continuous waveform during the stimulation periods. The Number of Cycles parameter, used in the command, determines this repetition count of the desired waveform for the stimulation session. To have a desired frequency, we have designed a delay function block to adjust the interval between samples of the stimulating waveform passing through the D/A converter. In this design the exact delay is represented by an integer variable that practically puts a limit on the maximum possible frequency. In addition, the maximum frequency is further limited by the operating clock of the D/A converter. In our SDBS design, with a D/A converter running with $1 \mathrm{MHz}$ clock frequency, the electrode can generate waveforms with the maximum frequency of $39215 \mathrm{~Hz} \cong 3.9 \mathrm{KHz}$ with $\pm 2 \%$ error. In addition, the reference voltage of the DAC is chosen to be $2560 \mathrm{mV}$ and the input data sample width of the sigma-delta modulator is set to be 8 bits. As a result, the maximum achievable output waveform amplitude is about $2560 \mathrm{mV}$ with a resolution of $2560 / 2^{\wedge}(\mathrm{N}=8)=10 \mathrm{mV}$. In this design the maximum value for the amplitude and frequency is set to $2500 \mathrm{mV}(0 \times 9 \mathrm{C} 4 \mathrm{mV})$ and $2.5 \mathrm{KHz}(0 \times 9 \mathrm{C} 4 \mathrm{KHz})$ respectively. In the data-based waveform generation scheme shown in Fig. 25(c), the stimulation waveforms are generated directly from the instantaneous amplitude samples transmitted by the external controller. For this approach, in addition to the amplitude data sequence, the controller also 
need to send a few other parameters such as sampling interval and number of waveform cycles to give a complete description of the stimulation waveform. During the waveform generation process, once the command base receives the command, along with all the necessary data and parameters, the received waveform data are fetched, aligned, shaped and fed to the DAC with the exact sampling interval that is required by the external controller. The stored waveform can be repetitively generated to increase the total stimulation time. Fig. 25(c) shows the schematic block diagram of the data-based generation scheme.

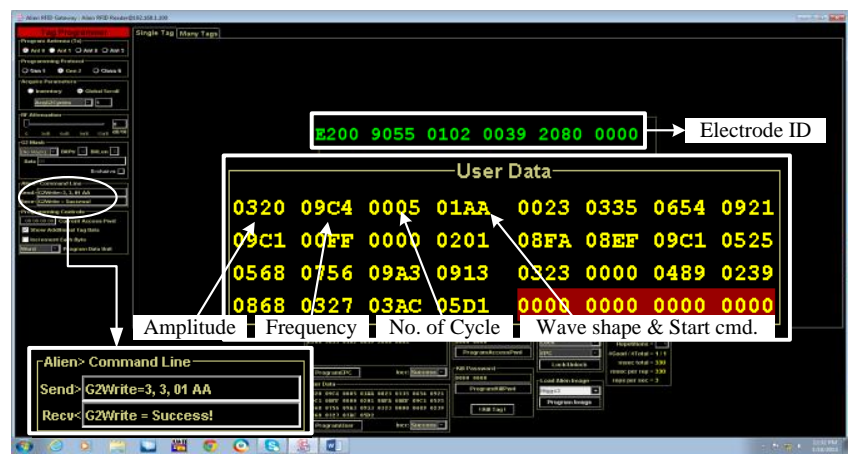

Figure 30: The command shown on the backend server screen is executed for waveform generation using the command-based approach.

\subsubsection{Neural Activity Recording \& Processing Module}

Fig. 26 shows the schematic block diagram of the neuro recording \& processing module in the SDBS system. It consists of a pre-processing unit (PPU) that includes an active prescalar, a low-pass filter and an analog-digital converter (ADC); and a control and 


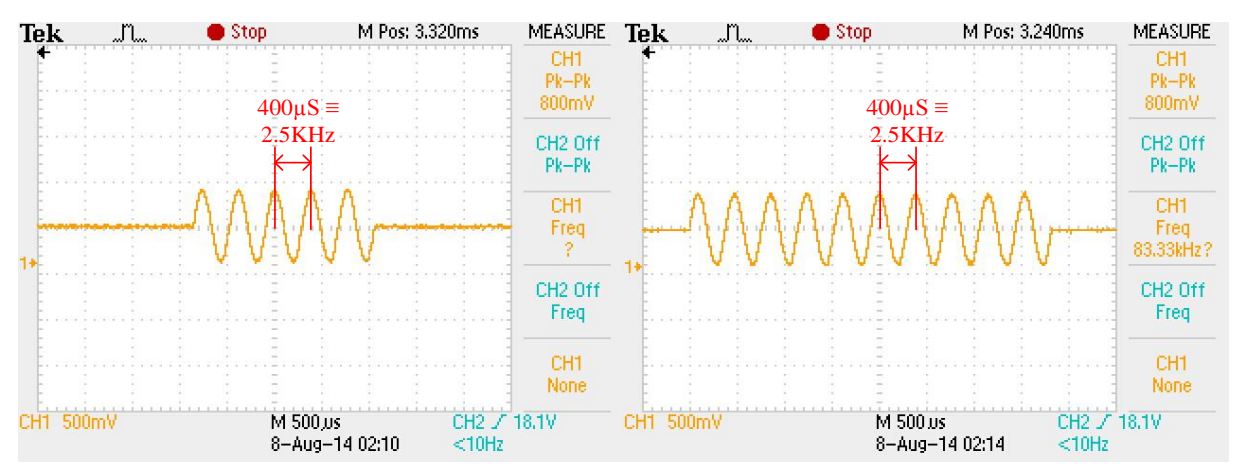

(a)

(b)

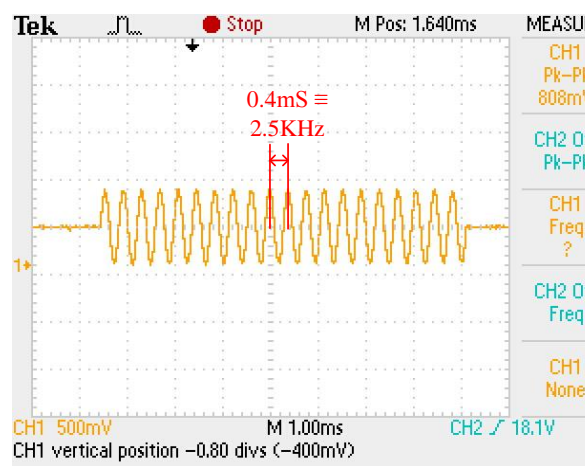

(c)

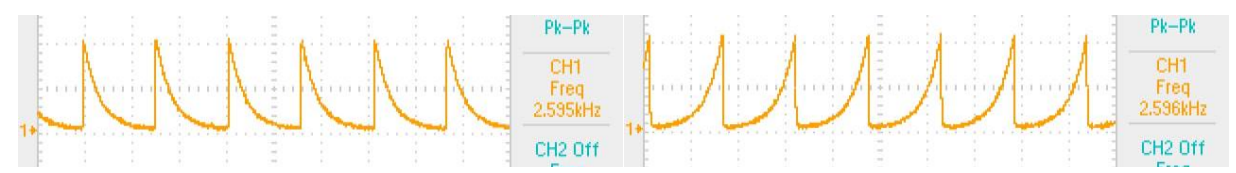

(e)

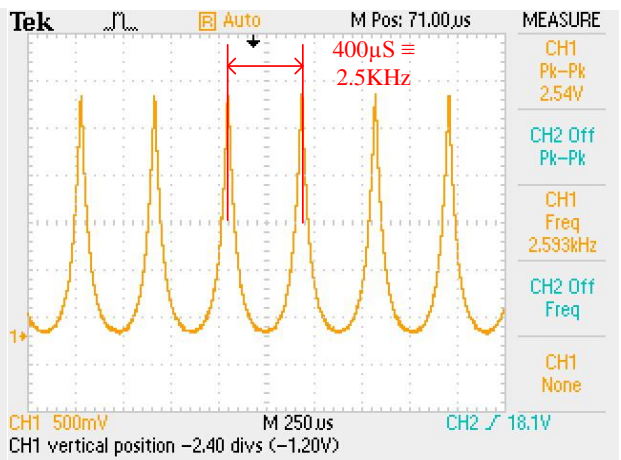

(d)

Figure 31: Smart electrode generated stimulation waveform output; (a) a sinusoidal wave with amplitude $800 \mathrm{mV}$, frequency $2.5 \mathrm{KHz}$ and No. of cycle 5 ; (b) a sinusoidal wave with amplitude $800 \mathrm{mV}$, frequency $2.5 \mathrm{KHz}$ and No. of cycle 10; (c) a sinusoidal wave with an amplitude of $800 \mathrm{mV}$, a frequency of $2.5 \mathrm{KHz}$ and the number of cycles equal to20; d) a Gaussian pulse; (e) negative exponential wave; and (f) a positive exponential wave.

processing unit (CPU) that provides the recorded data a temporary storage and implements some light-weight signal processing algorithms for feature detection, stimulation evaluation and the best waveform optimization. This module is designed to record the local field potential (LFP) of the neural activity. LFP signals typically are continuous, low frequency signals with frequencies less that $250 \mathrm{~Hz}$, and the amplitude up to $5 \mathrm{mV}[102$, 103]. In our design, the CPU's built-in ADC is used to convert the analog brain LFP signal 


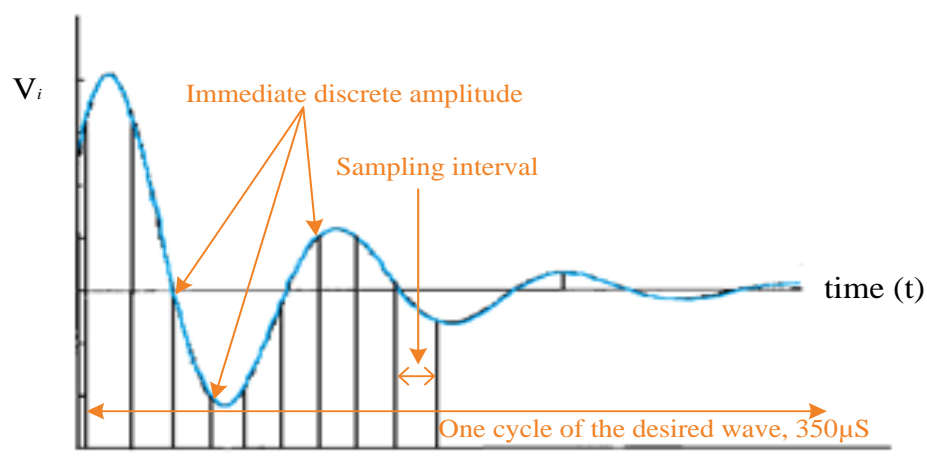

(a)

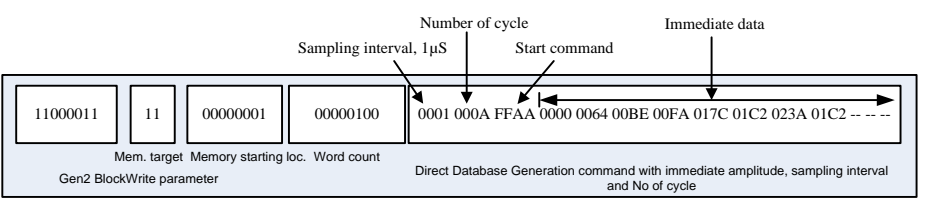

(b)

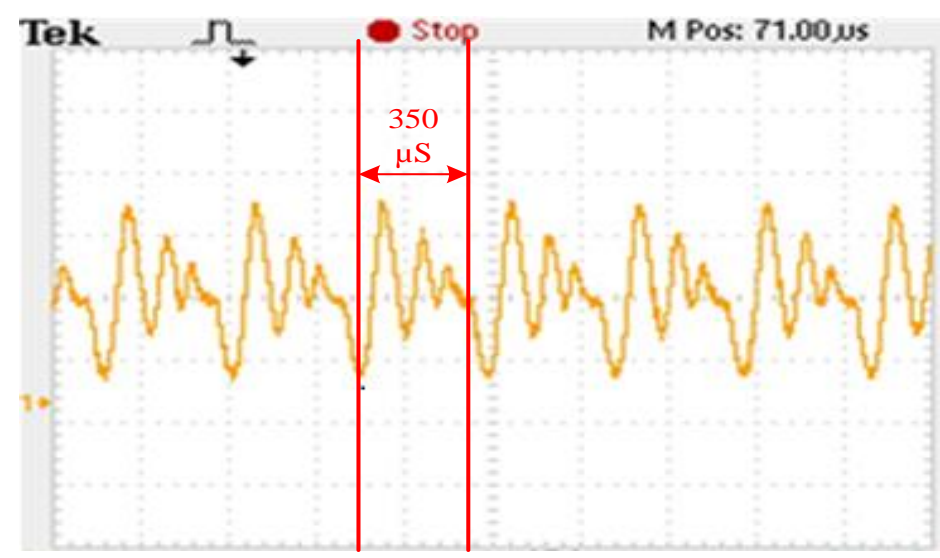

(c)

Figure 32: Data-based arbitrary waveform generation; (a) the ideal stimulating waveform to be generated; (b) the schematic structure of the data-based generation command; (c) the actual SBDSgenerated waveform.

to digital signals. The reference voltage and the resolution of the ADC is set to be $2.56 \mathrm{~V}$ and 8-bit, respectively. The ADC is capable of sampling and digitalizing voltage change as small as $10 \mathrm{mV}$. To further improve ADC sensitivity to voltage change, we have the ADC preceded by a pre-scalar with a gain of about 450 . With consideration of the gain of the pre-scalar, the $\mathrm{ADC}$ is capable of representing the voltage change as small as $22.2 \mu \mathrm{V}$. 
To pick the commensurate sampling frequency, we need make a tradeoff between the quality of data recording and the required memory storage space in the electrode. Higher sampling frequency ensures good quality of data recording, but requires more local memory space for data storage. On the other hand, the sampling rate for ADC has to satisfy the minimum Nyquist sampling rate to keep the relevant signal information. Therefore, in this work the sampling frequency was chosen to be $1.8 \mathrm{kbps}$. In the prototype system a $128 \mathrm{~KB}$ local memory space has been reserved in the electrode to save the recorded brain

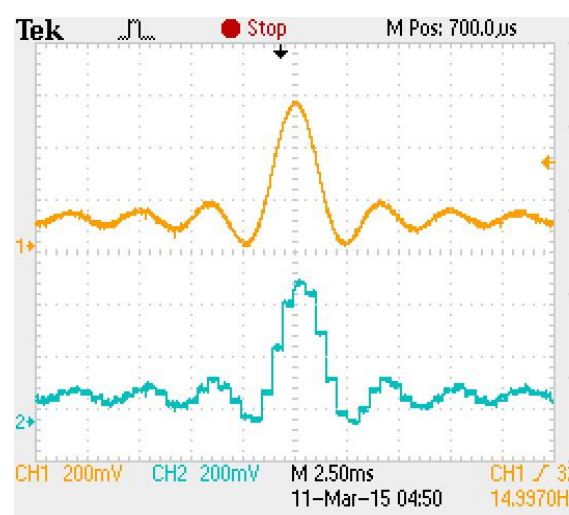

(a)

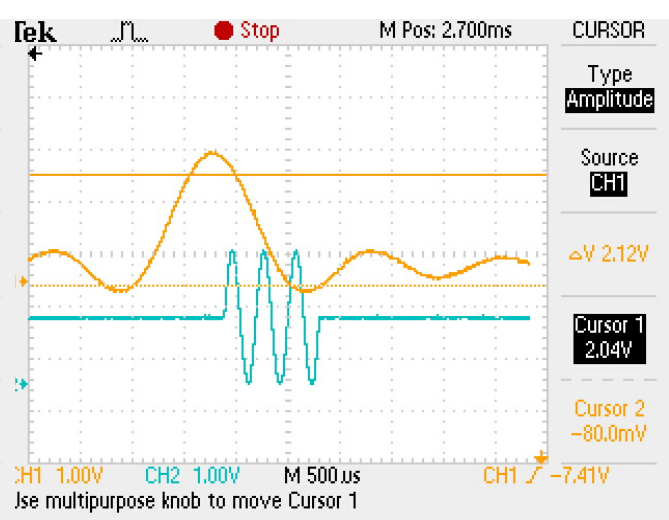

(b)

Figure 33: SDBS recording and processing module testing results: (a) The input waveform to the SDBS recording module and the waveform regenerated from the recorded data; and (b) SDBS threshold-based detection and stimulation generated from the SDBS closed-loop operation (for better representation, input signal wave is shown after the pre-scalar).

LFP data. The memory space can store upto128K samples of the instantaneous recorded data before it runs out of space in the memory. With the sampling rate set to be $1.8 \mathrm{kbps}$, it takes approximately $1.25 \mathrm{~min}$ for the memory space to be filled up. In the designed system, the control unit in the electrode monitors the memory space availability and informs the external controller about any upcoming issues. Then it is up to the external controller's decision whether it wants to transfer the data from the electrode for further analysis. If it 
does not, the electrode saves the maximum, minimum and the average of the recorded data of that particular session and empties the rest of the memory, for the next session. In this fashion, it saves a certain number of sessions' information (0xFF sessions) before it completely resets itself.

To demonstrate the feasibility of the closed-loop operational capability of the SDBS platform, in this prototype system, we have implemented a very simple threshold-based detection method. In the design, the reception of a signal spike with amplitude surpassing the threshold of $5 \mathrm{mV}$ is considered to the detection of the onset of a neural disorder such as excessive pathological motor neural firing in the PD's patients. In response, the processing module interrupts the waveform generation module to trigger the DBS operation. In the meantime, the feedback neural data are recorded and evaluated to determine the further actions for the next step. In addition, the smart electrode flags the event and informs the backend controller (i.e. using modified ID transmission, Table VIII). In the results section, a testing case of the closed-loop detection and waveform generation will be presented. However, the above described procedure of SDBS operation was just a very simple instance of demonstrating the SDBS's ability in performing closed-loop detection and stimulation waveform generation. The more advanced signal processing and data analysis algorithms can be implemented and applied to improve its performance further more.

\subsubsection{Data Security Module}

Traditional DBS implemented devices could be maliciously programed by unauthorized users or intruders due to their lack of security measures. To address the security 
vulnerability issue of the current DBS systems, we came up with a comprehensive approach involving both electrodes and external controller. Specifically, the security measures implemented in the SDBS are detailed as follows. (i) External controller identity verification. When the external controller tries to gain access to the electrode, it has to provide the verification code/passcode accompanied with the user verification code first. The verification code can be set by the system administer. Subsequently, any access to the electrode is only allowed after successful verification of right combination of the codes. (ii) Firmware-level data encryption/decryption. Once the electrode starts communication with the verified controller, the system is still very vulnerable to eavesdropping by any unauthorized devices or intruders. To protect the SDBS communication from this kind of attack, we have implemented a simple XOR-ed encryption using a 16-bit encryption key. The key is not transmitted with the data, instead it was preprogrammed inside the electrodes and the associated controllers by the authorized system administer during the system installation or anytime they want to reset or reinstall it.

\subsubsection{SDBS Powering and Energy Harvesting}

According to the design the analog frontend is fully passive. The modulation/demodulation circuitry runs by employing back scattering modulation and ASK demodulation technique in compliance with EPCGen2 Protocol standard [69]. It also has a 5-stage CockcroftWalton voltage multiplier circuitry working as RF energy harvester. Fig. 27 shows the

energy harvesting module and associated measured data graph. At an optimal distance of $1.4 \mathrm{~m}$, the harvester produces approximately $310 \mu \mathrm{W}$. The harvested power is mostly spent to run the signal conditioning unite (LMV301) which requires $270 \mu \mathrm{W}$, and also helps 
recharging the battery of the processing core, which is powered by a $3.0-\mathrm{V} \mathrm{MnO}(\mathrm{Li}+)$ coin battery (CR2354). With the processor running at $80 \mathrm{MHz}$, the average power consumption is $(50 \times 70 \mu \mathrm{A} \times 3 \mathrm{~V}) 10.5 \mathrm{~mW}$ in full running mode, and $(50 \times 8 \mu \mathrm{A} \times 3 \mathrm{~V}) 1.2 \mathrm{~mW}$ in low power mode. However, the electrode has the capability to deliver stimulatory waveforms with maximum power up to $135 \mathrm{~mW}$. The measurement is performed across a $50 \Omega$ resistance (typical human brain tissue resistance). Table IX shows a comparative study of power consumption of few contemporary DBS platforms.

\subsection{SDBS Testing and Evaluation}

To verify the technical feasibility of the proposed SDBS electrode, we have built a complete SDBS prototype system in the laboratory. The prototype system was further tested and evaluated. Fig. 28 shows a test bench arrangement to test the prototype SDBS system and its physical components. In this system, a RFID (ALR-9650) is used as the external controller that also works as a gateway between the smart electrode and the backend server through the internet cloud. For commanding and communicating with the SDBS electrodes the ALR-9650 built-in software interface was adopted in the backend computer. The system, being built on RFID communication protocol, it assigns a unique ID to each of the electrodes which has been leveraged for targeting and selecting process in a single/multi-electrodes environment. Fig. 29 shows the SDBS' communication module performance in the single and multi-electrode environment. The detection rate of the electrode declines with the increase of the physical distance between the external controller and the electrode, as well as the number of electrode is increased in the vicinity. The following subsections include few testing results obtained from the SDBS system. 


\subsubsection{Waveform Generation}

Command-Based Waveform Generation: For command-based waveform generation to be effective, the associated command should be first generated and then sent from the backend controller to the electrode. The following testing case is implemented in the prototype system to generate a sinusoidal stimulation waveform with the required parameters specified. The testing sinusoidal waveform to be generated with the amplitude, frequency, and number of the waveform cycles set to be $800 \mathrm{mV}, 2.5 \mathrm{KHz}$, and 0005 , respectively. We executed the following waveform generation commands from the SDBS external controller: G2Write=3, 0, 0320 (Amplitude set to 800mV) G2Write=3, 1, 09C4 (Frequency set to $2.5 \mathrm{kHz}$ ) G2Write=3, 2, 0005 (No. of waveform cycles set to 0005) G2Write=3, 3, 01 AA (Waveform type set to be sinusoids) The parameters have been programmed on one-by-one basis individually, as shown in Fig. 30. In this case, the 4th command (G2Write=3, 3, 01 AA) is the start command that instructs the electrode to generate the stimulation waveform with the associated parameters set above. Fig.31 (a) shows the generated output waveform corresponds to the commands sent above, and the rest of the figures show a few other waveforms that we have generated from the smart electrode.

Data-Based Arbitrary Waveform Generation: Fig. 32 depicts a testing case for data-based waveform generation. If we need to generate a waveform for stimulation like shown in Fig. 32 (a), we can use the data-based waveform generation scheme in SDBS. The desired one wave duration has been chosen to be $350 \mu \mathrm{S}$. We have sampled the signal in 35 instantaneous amplitudes as listed in Table X. To command the electrode to generate such waveform, we can program a data-base generation command shown in Fig. 32 (b) from the 
external controller. The generated output waveform is shown in Fig. 32 (c). The duration of the generated signal was measured as $350.1 \mu \mathrm{S}$ and the maximum amplitude is measured as $562 \mathrm{mV}$ that is well inside the $2 \%$ error limit.

\subsubsection{Recording and Processing Module Testing}

To test the recording functions of the prototype SDBS system, we have provided a known signal at the sensory contact for the electrode to record and save it; and further used the saved data as the data samples to regenerate it. Results shows that the regenerated received signal from the external controller was very close to the known signal provided at the sensory contact. This innovative experimental setup can be used to verify how close the waveform generated by the databased approach is to the original recorded waveform. If these two waveforms are the same or close, it would demonstrate that both the data recording and the data-based waveform generation modules in the SDBS prototype system function normally and well. In the testing, the original waveform recorded at the sensory contact and the one generated from the data-based approach are shown in the top and bottom part of Fig. 33 (a), respectively. The two waveforms are observed to be in very close agreement, which demonstrates that the SDBS waveform recording and the databased waveform generation functions work well. The slight disparity between the two waveforms is due to the quantization nonlinearities, i.e. the differential nonlinearity DNL in $\mathrm{A} / \mathrm{D}$ converter and the integral nonlinearity INL in D/A converter. The waveform difference can be minimized by improving the DNL and INL factors of the A/D and D/A converters. 
To test the processing module and the closed-loop operation of the SDBS prototype, we designed a special waveform with a spike and fed it the electrode contact for recording and analysis. The testing performed by the electrode is to detect if the recorded signal surpasses a pre-selected threshold at any moment. If an above-threshold signal is detected, a sinusoid waveform is automatically generated for brain stimulation. If no more above-threshold signal is detected for a certain period of time after stimulation is triggered, the stimulation will be stopped. Therefore, this setup is a simple closed-loop operation for the SDBS. In our testing, the amplitude of the spike in the testing waveform is $5.1 \mathrm{mV}$ and the detection threshold is chosen to be $5 \mathrm{mv}$. Because the threshold is crossed in the testing, the SBDS electrode is triggered to generate a sinusoidal waveform lasting for $0.75 \mathrm{~ms}$ with amplitude of $2.5 \mathrm{~V}$ and the period of $0.25 \mathrm{~ms}$, shown as the blue plot in Fig 32 (b). However, more advanced signal processing and data analysis algorithm could be applied to the recorded neural waveform and designing the optimal stimulation waveform accordingly with the SDBS platform to achieve more effective closed-loop operation.

\subsection{Conclusions}

This article introduced the concept and architecture of a novel implantable device, termed as smart deep brain stimulators (SDBS). A prototype system of the proposed SDBS has been designed and developed for testing and performance evaluation. The SDBS systems can achieve unprecedented "smartness" and "power efficiency" by embedding a microprocessor inside the implantable electrodes with significant computing power and the passive wireless connection to the external controller, and further to more powerful and resourceful remote servers through the Internet. The basic functions of the designed SDBS 
include neural activity recording, signal processing and data analysis, stimulation waveform optimization and generation. The best stimulation waveform can be optimized, generated and applied based on the evaluation result of the real-time recorded neural activity signals. With the prototype system successfully designed, developed and tested in this work, the SDBS can be used as a general platform to implement fully adaptive closedloop DBS. Furthermore, the SDBS electrode is ready to be fabricated into a single implantable integrated-circuit (IC) chip to greatly enhance its reliability, security, lowpower survivability and integration level. The new device is capable to become the next generation standard internet-connected smart BMBI devices for future brain research. 
CHAPTER V

DESIGN AND DEVELOPMENT OF WIRELESS ECG SENSOR 


\subsection{Introduction}

Cardiovascular diseases the leading global cause of death, now-a-days. According to the 2015 Heart Disease and Stroke Statistics Update by American Heart association it accounting for 17.3 million death per year which exhibits an alarming potential to grow up to 23.6 million by 2030 . Estimated by the same agency about 85.6 million American are suffering from some degree of cardiovascular diseases or the after-effect of stroke, and living in an increased risk of cardiac failure [104]. Amongst, people those who have already survived from previous cardiac arrest are in increased risk of sudden death causing from sudden cardiac failure happening again. However, early detection and diagnosis of any cardiac anomaly through continuous heart monitoring can reduce this risk by many fold. Electrocardiogram (ECG) is an advanced diagnostic system that capture the heart activity and converts it in to the electrical pulses and bring the blue print of heart activity in visual, is a very effective way of detecting anomaly in the heart functionality. To date, it has been working as a very efficient tools for diagnosis $[105,106]$. Due to the advancement of telemedicine, eHealth and smart-wearables, ECG monitoring is entering to the realm, where smart ECG monitoring device can acquire patient cardiac data and send it to the high end remote server for advanced monitoring and critical alarm generation [107-109].

In smart ECG systems, data acquisition circuitries are embedded into a wireless sensory system, might form a standardized Body Area Network (BAN) with other wearable sensors, and connect to the cloud via a smart getaway. In developing those systems, one of the prime design goals is to make them as simple as possible to make it convenient for the patient to carry it and to roam around in a daily life situations. 
Advanced remote monitoring solution using telecommunication technology for ECG diagnosis is getting very popular nowadays. The EPI-Medics project [110] demonstrates an ambiance intelligent system that monitors the patient's cardiac activity through a Personal ECG Monitor (PEM) system and maintains communication with the remote healthcare facility via Bluetooth and GSM/SMS technology. Morrison el al. [111] presented a smart shirt with embedded wireless ECG monitoring system. This system is useful for monitoring people going through strenuous activity, like while performing athletic activity. However as a continuous wearable monitoring system, considering the form filling textile architecture, the proposed system is not convenient.

In addition, considering the power consumption, most of the solutions, like few presented above, have used the standard ISM band radio, like Bluetooth, ZigBee, and/or other active technology as the wireless backbone between the on-body system and the off-body computational unit. These type of wireless protocols are relatively power hungry protocols with compared to the available resource in a wearable situation. Now-a-days, one of the prime focuses in smart wearable design is to reduce the power consumption as much as possible or even make it an energy sustainable system that can run by the harvested energy. The use of these technologies for today's wearables hinders the process and might be impractical. Therefore, the new efficient powering technology needs to be discovered for the SBMBI applications.

In an effort to reduce power consumption, Cheng-Pin Wang et al. [112] presented a semipassive wireless ECG monitoring system. It this solution, they have used UHF RFID protocol to as a communication module between the wearable sensor and the off-body 
computer, thereby reducing the dominant power consumption factor coming from the communication module in regular solutions. However in this presented solution the ECG signal acquisition frontend is sequentially executable unit that acquires data every time the communication module is brought into the "Query" state by the questing RFID reader. This limits the system's data acquisition rate which completely dependent on the handshaking process between the tag system and the reader.

To address this issue, we have incorporated our SBMBI communication interface with the

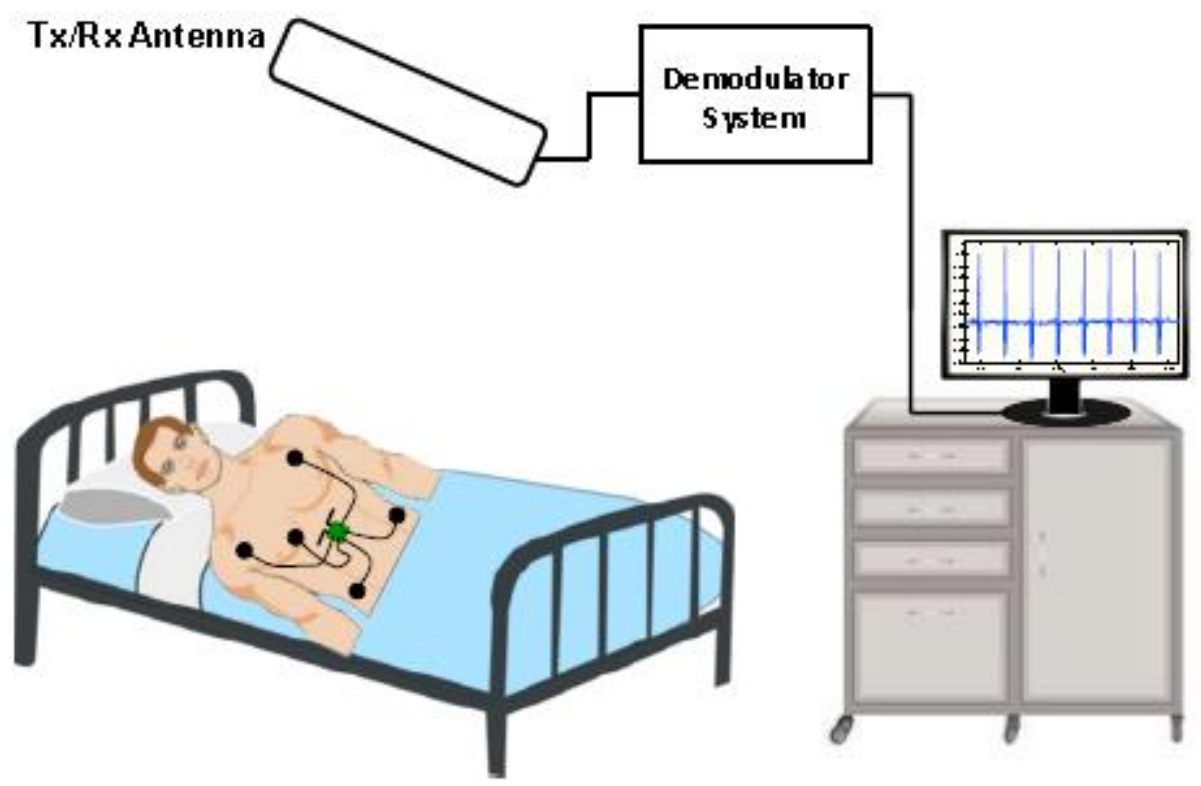

Figure 34: Schematic of the proposed ECG monitoring system in real-life deployment situation [113].

standard ECG acquisition system in which data acquisition is completely a parallel process with the communication module thereby making the ECG data actuation system independent and reliable. Fig. 34 shows a schematic of the proposed ECG monitoring system in real-life deployment situation. 


\subsection{System Architecture:}

The Electrocardiogram (ECG), a common signal found in most of the clinical settings, measures the electrical activities related to the cardiac operation in vivo. It is relatively large for a bio-signal (1 $\mathrm{mV}$ peak-to-peak) with frequency spectrum ranging from approximately $0.67 \mathrm{~Hz}$ to $100 \mathrm{~Hz}$ [114]. The rhythmic contraction of the pacemaker cells in the sinoatrial (SA) node, the atrioventricular (AV) node and the both ventricles operate in a coordinated sequence. The coordination of this process results in a well-defined electric field named as "PQRST" complexes which works as the signature of cardiac operation. This electric field can be measured in time domain through a non-invasive, three-electrode system, known as "Einthoven's triangle" method. The differential among electrodes on the Left Arm, Left Leg, and the Right Arm form the three sides of the Einthoven's triangle while the Right leg is used as reference. Each electrode has a unique spatial perspective of the heart and each holds specific diagnostic information. In this study, we have used "Lead I" as it only requires electrodes on the arms and right leg.

The electrodes on the arms and reference leg are standard Silver-Silver Chloride $(\mathrm{Ag} / \mathrm{AgCl})$ electrodes from the company ORI. An AD620AN instrumentation amplifier from Analog Devices was selected for its low bias current and high common-mode rejection ratio. The difference from the left arm to the right arm was taken as the input then amplified by a gain of $101 \mathrm{~V} / \mathrm{V}$, while the reference was connected to the leg which was powered at $\mathrm{Vcc} / 2$. The output from the instrumentation amplifier was sent through passive low-pass $(106 \mathrm{~Hz})$, twin-t notch $(60 \mathrm{~Hz})$, and high-pass $(0.4 \mathrm{~Hz})$ filters to reduce high frequency muscle noise, ambient $60 \mathrm{~Hz}$ noise, and low-frequency drift due to respiration, respectively. The last step 
was amplification with an LM358 operational amplifier with $110 \mathrm{~V} / \mathrm{V}$ gain to place the filtered signal into the $0-2.5 \mathrm{~V}$ range. Figure 34 shows a schematic system diagram of the proposed system and Fig. 35 shows the schematic of the ECG data acquisition module.

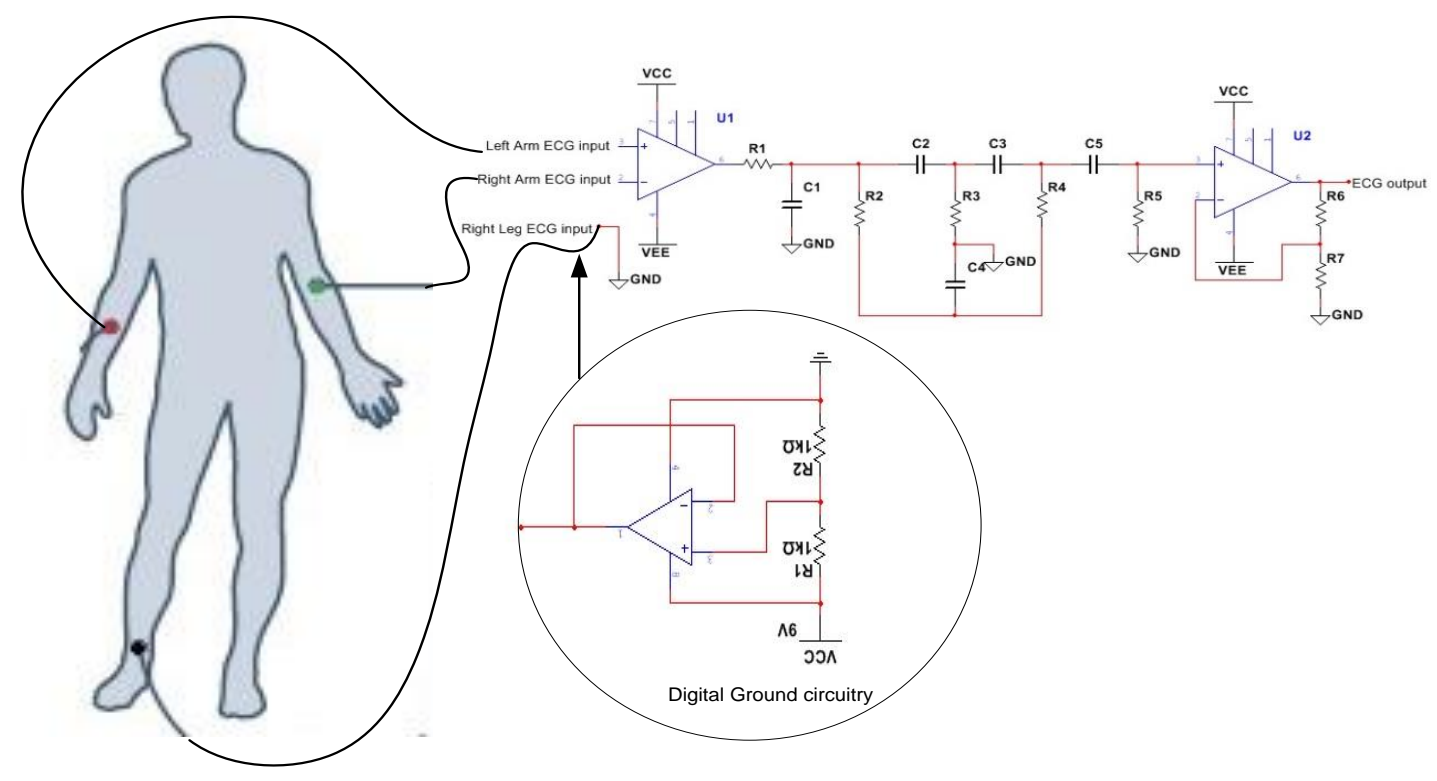

Figure 35: Schematic diagram of the ECG signal acquisition circuitry.

A Graphical User Interface (GUI) is also designed to run the sensor system, acquire data wirelessly and represent the graphical view of the acquired data on the GUI screen. The implemented system also store the instantaneous data point in a spreadsheet for further analysis.

\subsection{Results}

Figure 36 shows the ECG data actuation test bench, collecting data from the test subject, and Fig. 37 shows the screenshot of the implemented GUI capturing ECG signal from the SBMBI platform connected to the subject body. Finally the database values were plotted 


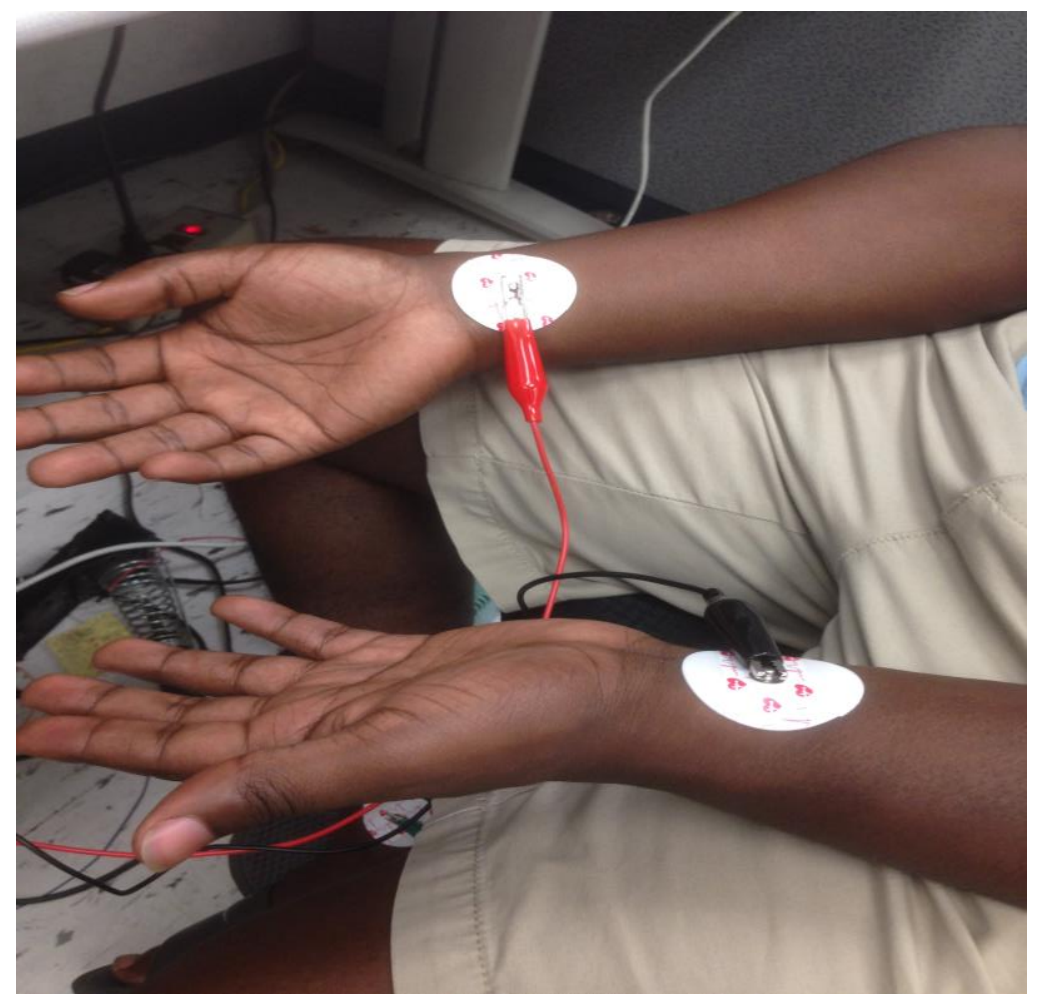

Figure 36: ECG data acquisition test bench

to verify the integrity of the recorded signal. Visual comparison of the plotted signal with a standard ECG signal, as presented in Fig. 38, shows a closer coherence thereby verifying the performance of the implemented system. This representation is deemed to be adequate enough to monitor a patient's cardiac activity remotely. However rigorous clinical trial is required to verify signal integrity and reliability.

\subsection{Discussion}

In this wireless ECG monitoring system design, Einthoven's triangle 3-electrode systems has been implemented. According to this model, two electrodes separated by approximately 0.3 meter (LA to RA) are used for the actual differential recording which is known as Lead I. The third electrode is used only for noise suppression (ground lead), 


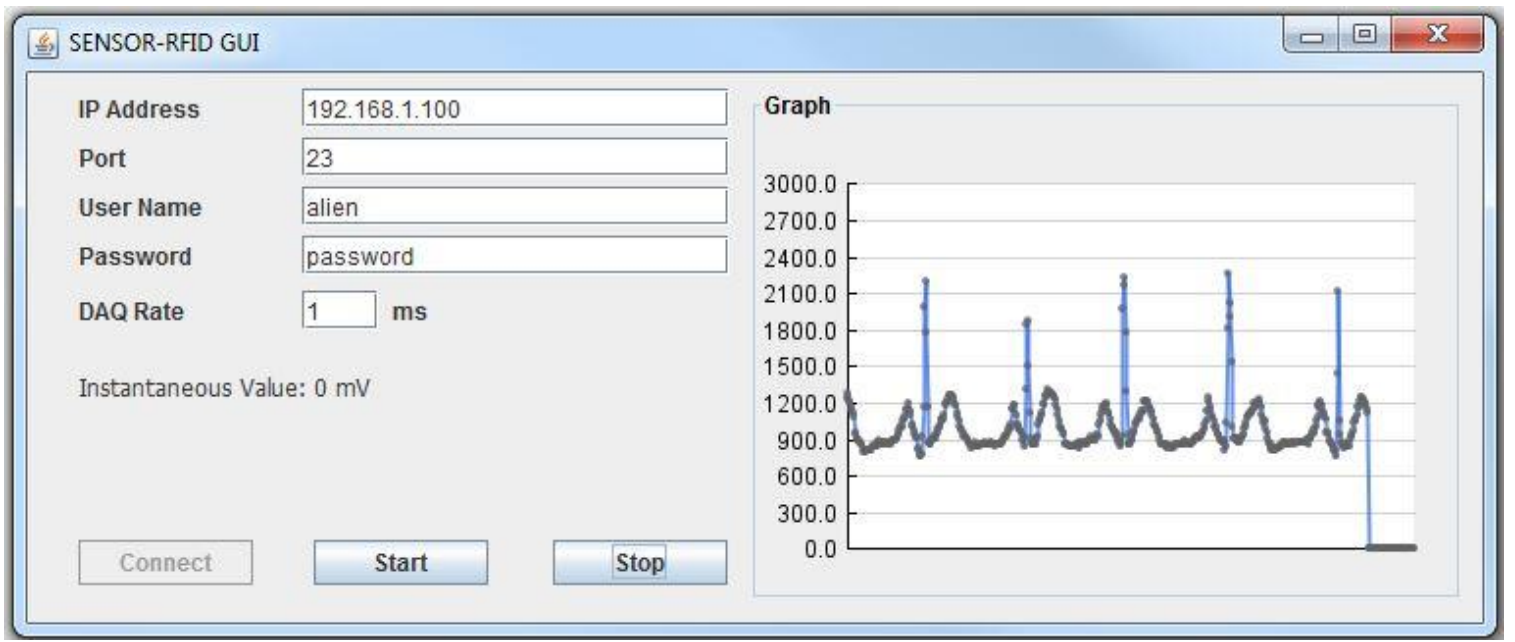

(a)

Wireless ECG Monitor

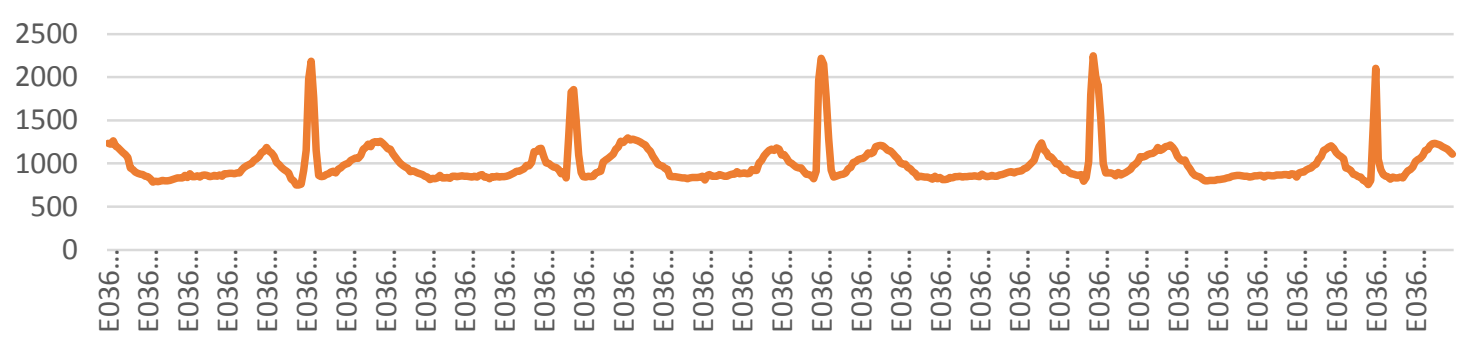

(b)

Figure 37: Wireless ECG monitoring interface; (a) Graphical User Interface (GUI) for the wireless ECG monitoring system; (b) Reconstructed ECG signal from the backend computer data-base

placed in the right leg. The reference ground connection serves as a noise suppressor in the system. Without this reference electrode, system becomes vulnerable to artifact causing from involuntary muscle movements. In article [115] Thakor et al. found that a twoelectrode design could be useful for portable Holter monitors, and port-able arrhythmia monitors. Our solution is in some ways similar to this model other than the reference ground connection. The ECG-signal obtained through their system differs in some ways from a standard Lead I recording as it only uses two electrodes that are placed close to each 


\section{Wireless ECG Monitor}

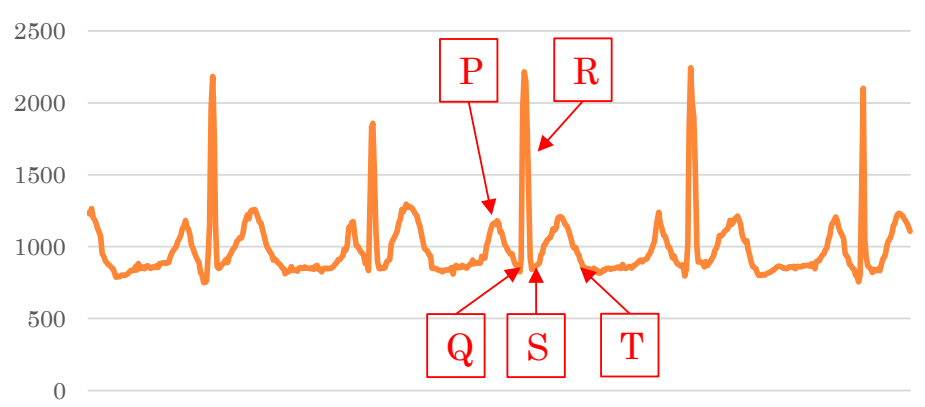

(a)

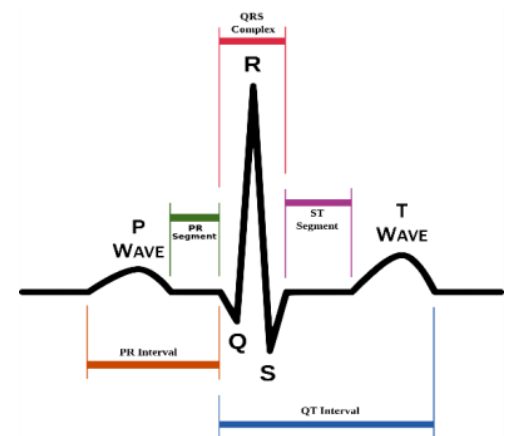

(b)

Figure 38: (a) The "PQRST" complexes in the acquired signal; (b) A standard signal with the "PQRST" complexes

other, which, therefore, required further investigation before using this recording principle for diagnostic purposes. With compared to this system, due to the presence of reference ground electrode, signal acquired through our system exhibits a closer coherence with the standard Lead I signal. Figure 38 presents a comparative study between a signal collected through SBMBI platform and a standard ECG signal. Even though proper clinical trials are clearly needed to verify the integrity of the recorded signal, it seems reasonable to assume that our new ECG-monitoring system will be able to reliably monitor a patient cardiac activities.

In our design, we have used dry electrode as the ECG transducer. The common alternative to this is a wet electrode system, where an electrolytic gel is used at the surface of the electrode to increase the conductivity of the electrode. Even though hypothesis [116] claims that there is no significant difference found in using either dry or wet electrode system in ECG data acquisition, wet electrode system suffers from few inherent shortcomings while using it for a relatively longer period of time. The major limitations include, potential deterioration of signal quality due to the dehydration of the get on the 
electrode surface, irritation and swelling of the associated tissue, etc. In addition, the potential for electrolytic gel dehydration also implies that this type of electrodes are subjected to expiration at some point of time from the date of production.

Considering all these, the use of dry electrodes based efficient ECG data acquisition frontend and the use of low power SBMBI platform for wireless transmission makes this implemented system suitable for longtime use by any outpatient. However, the system is implemented in a prototype board using off-the-self component which makes the unit too large for real-life day-to-day basis application. Therefore, an ASIC or system on a chip (SOC) implementation could be an important future task.

\subsection{Conclusions}

Being designed on the SBMBI platform, this system can work as a continuous event recorder, which can be used to follow up patients who have survived cardiac arrest, cardiac syncope or ventricular tachycardia. Since SBMBI is capable of connecting to cloud, along with in hospital monitoring, this system could be used in remote patient monitoring, i.e. suitable for outpatient scenario. This system is designed to ensure used comfort while carrying the sensor for a longer period of time. Use of dry electrode has made it suitable for several days use in a row before it is required to replace it with a new one. We hope that our implemented system will provide the application developer with a suitable platform for next generation remote ECG monitoring thereby offering improved patient care both in/outside the hospital. 


\section{CHAPTER VI}

CONCLUSION AND FUTURE WORK 


\subsection{Conclusion}

Brain-Machine-Brain-Interface (BMBI) is the next generation research. A suitable BMBI module will offer a broad way for the researcher and the industry to explore deeper into the brain. At the same time BMBI devices needs to take the advantage of powerful cloud computation, access the big data stored in the remote server to take the optimum decision on the acquired information. Having the capability of accessing the remote cloud will leverage the BMBI system effectiveness hence making it a smart system.

In this research work we have designed, prototyped and tested a Smart Brain-MachineBrain-Interface (SBMBI) the employs a fully passive wireless communication to establish a communication between the brain and machine and at the same time it can communicate with the cloud via an external controller. In SBMBI the communication module designed based on the RFID Gen2 protocol standard, which is a very light weight power efficient protocol. Standard RFID Protocol has been modified and redefined to make it execute user level command and convey user level non-RFID Standard data. However the protocol was modified in a way so that any Standard Gen2 RFID reader can be used as the interface between the SBMBI and external smart devices. This implemented communication module is fully passive - it does not require any power from the on body BMBI module, instead it runs by the harvested power from the external controller. Hence incorporation of this novel technique as the communication backbone for the SBMBI, made it a very power efficient device which is very suitable next generation wearable devices.

The effectiveness of the SBMBI is tested in different scenarios. A Smart Deep Brain Stimulation (SDBS) electrode System is developed based on the principle of the SBMBI 
interface, where the SDBS electrode can monitor the brain activity and generate different stimulation signal and also capable take advantage of the remote cloud through the backend controller. In addition, the designed SDBS system has leveraged the existing DBS system by incorporating a novel mechanism for arbitrary wave form generation. Along with the existing regular wave forms, this incorporation of arbitrary wave generation mechanism, which will offer the DBS physicians with a wide variety of options available to explore the yet to understand neural functionalities.

\subsection{Future Work}

Based on the results presented in this thesis, one can find several further research topics worth further investigation.

\subsubsection{Design and Fabrication of SDBS Microchip}

In this thesis a novel concept of Smart Deep Brain Stimulator is presented with is prototype design and laboratory testing. However the future work focus on implementing the SDBS circuitry into an Application Specific Integrated Circuit (ASIC) to make it suitable for in vivo testing. In this regard future work includes CAD design, simulation, layout design and floor planning of the associate circuitry, and fabricate the SDBS as a single microchip.

\subsubsection{Investigate the Feasibility of a fully passive SDBS system}

Like any other wearable device, the ultimate goal for the SBMBI/SDBS is to make it as power efficient as possible. Current design has presented the SDBS as a semi-passive 
device, where the communication module is passive (run by the RF harvested power) and the digital processing core is battery assisted.

The future work should focus to increase the efficiency of the RF energy power along with exploring the feasibility of other type of energy harvester, i.e. thermal energy harvester. At the same time, attention should be paid in designing of the SBMBI ASIC to make it an efficient low power device so that the SBMBI system becomes a fully passive system.

\subsubsection{Performing in vivo Test using the SDBS system}

Since SBMBI is aimed to be a smart interface between a living beings and machine it's important to in vivo test on the designed system. Once the SBMBI is fabricated as a microchip, future work includes implanting it microchip into a test animal, following proper regulation, and analyze the performance of the designed system. 


\section{REFERENCES}

1. Vidal, JJ (1973). "Toward direct brain-computer communication". Annual Review of Biophysics and Bioengineering. 2 (1): 157-80.

2. Y. Chen, E. Yao and A. Basu, "A 128-Channel Extreme Learning Machine-Based Neural Decoder for Brain Machine Interfaces," in IEEE Transactions on Biomedical Circuits and Systems, vol. 10, no. 3, pp. 679-692, June 2016.

3. S. Lee, S. C. Yen, L. D. Liao, G. G. L. Gammad, N. V. Thakor and C. Lee, "Flexible sling electrode for bidirectional neural signal recording and selective stimulation," 2016 IEEE 29th International Conference on Micro Electro Mechanical Systems (MEMS), Shanghai, 2016, pp. 375-378.

4. J. N. Y. Aziz et al., "256-Channel Neural Recording and Delta Compression Microsystem With 3D Electrodes," in IEEE Journal of Solid-State Circuits, vol. 44, no. 3, pp. 995-1005, March 2009.

5. X. Liu, V. Valente, Z. Zong, D. Jiang, N. Donaldson and A. Demosthenous, "An Implantable Stimulator With Safety Sensors in Standard CMOS Process for Active Books," in IEEE Sensors Journal, vol. 16, no. 19, pp. 7161-7172, Oct.1, 2016.

6. A. T. Connolly et al., "A Novel Lead Design for Modulation and Sensing of Deep Brain Structures," in IEEE Transactions on Biomedical Engineering, vol. 63, no. 1, pp. 148157, Jan. 2016.

7. H. Bahrami; S. A. Mirbozorgi; A. T. Nguyen; B. Gosselin; L. A. Rusch, "System-Level Design of a Full-Duplex Wireless Transceiver for Brain-Machine Interfaces," in IEEE Transactions on Microwave Theory and Techniques , vol.PP, no.99, pp.1-10.

8. A. T. Avestruz et al., "A $5 \mathrm{Imu} \mathrm{W/Channel} \mathrm{Spectral} \mathrm{Analysis} \mathrm{IC} \mathrm{for} \mathrm{Chronic}$ Bidirectional Brain-Machine Interfaces," in IEEE Journal of Solid-State Circuits, vol. 43, no. 12, pp. 3006-3024, Dec. 2008.

9. Abdelhalim, Karim, et al. "915-MHz FSK/OOK wireless neural recording SoC with 64 mixed-signal FIR filters." IEEE Journal of Solid-State Circuits 48.10 (2013): 24782493.

10. K. Abdelhalim, V. Smolyakov and R. Genov, "Phase-Synchronization Early Epileptic Seizure Detector VLSI Architecture," in IEEE Transactions on Biomedical Circuits and Systems, vol. 5, no. 5, pp. 430-438, Oct. 2011. 
11. K. Abdelhalim, H. M. Jafari, L. Kokarovtseva, J. L. P. Velazquez and R. Genov, "Neural synchrony-monitoring wireless brain implant for intractable epilepsy neuromodulation," Neural Engineering (NER), 2013 6th International IEEE/EMBS Conference on, San Diego, CA, 2013, pp. 65-68.

12. W. Biederman et al., "A $4.78 \mathrm{~mm} 2$ Fully-Integrated Neuromodulation SoC Combining 64 Acquisition Channels With Digital Compression and Simultaneous Dual Stimulation," in IEEE Journal of Solid-State Circuits, vol. 50, no. 4, pp. 1038-1047, April 2015.

13. H. G. Rhew, J. Jeong, J. A. Fredenburg, S. Dodani, P. Patil and M. P. Flynn, "A wirelessly powered log-based closed-loop deep brain stimulation SoC with two-way wireless telemetry for treatment of neurological disorders," 2012 Symposium on VLSI Circuits (VLSIC), Honolulu, HI, 2012, pp. 70-71.

14. Z. T. Irwin et al., "Enabling Low-Power, Multi-Modal Neural Interfaces Through a Common, Low-Bandwidth Feature Space," in IEEE Transactions on Neural Systems and Rehabilitation Engineering, vol. 24, no. 5, pp. 521-531, May 2016.

15. Obeso, J. A., et al. "Surgery for Parkinson's disease." Journal of neurology, neurosurgery, and psychiatry 62.1 (1997).

16. Online, available at: http://www.medgadget.com/2009/02/medtronics_reclaim_deep_brain_stim_approved _for_some_ocd_sufferers.html.

17. Centonze, Diego, et al. "Distinct roles of D1 and D5 dopamine receptors in motor activity and striatal synaptic plasticity." The Journal of neuroscience 23.24 (2003): 8506-8512.

18. Johnson, W. Brad, and William L. Johnson. The Minister's Guide to Psychological Disorders and Treatments. Routledge, 2014.

19. Medtronic DBS Lead Kit for Deep Brain Stimulation. Available online: http://professional.medtronic.com/wcm/groups/mdtcom_sg/@mdt/@neuro/document s/documents/dbs-1d3387-9-impmanl.pdf

20. John, Michael Sasha. "Adaptive brain stimulation method and system." U.S. Patent No. 6,066,163. 23 May 2000. 
21. Abdelhalim, K., Jafari, H. M., Kokarovtseva, L., Perez Velazquez, J. L. and Genov, R. Neural synchrony-monitoring wireless brain implant for intractable epilepsy neuromodulation 2013

22. Abdelhalim, Karim, Genov, R. 915-MHz wireless 64-channel neural recording SoC with programmable mixed-signal FIR filters 2011.

23. Abdelhalim, Karim, Genov, R. 915-MHz wireless 64-channel neural recording SoC with programmable mixed-signal FIR filters.

24. Agarwal, R., Sarma, S. V. The effects of DBS patterns on basal ganglia activity and thalamic relay $2012 \mathrm{~S}$.

25. Chen, Y., Hao, H. W., Hu, Y. X. and Li, L. M. Wireless wrist-wearable wake/sleep identification device for closed-loop deep brain stimulation 2013.

26. Medtronic DBS Lead Kit for Deep Brain Stimulation. Available online: http://professional.medtronic.com/wcm/groups/mdtcom_sg/@mdt/@neuro/document s/documents/dbs-ld3387-9-impmanl.pdf

27. Hosain, M. K., Kouzani, A. Z., Tye, S. J., Abulseoud, O. A., Amiet, A., Galehdar, A., et al. (2014). Development of a compact rectenna for wireless powering of a headmountable deep brain stimulation device. Translational Engineering in Health and Medicine, IEEE Journal of, 2, 1-13.

28. Hua Gao, Walker, R. M., Nuyujukian, P., Makinwa, K. A. A., Shenoy, K. V., Murmann, B., et al. (2012). HermesE: A 96-channel full data rate direct neural interface in 0.13 m CMOS. Solid-State Circuits, IEEE Journal of, 47(4), 1043-1055.

29. Hyo-Gyuem Rhew, Jaehun Jeong, Fredenburg, J. A., Dodani, S., Patil, P., \& Flynn, M. P. (2012). A wirelessly powered log-based closed-loop deep brain stimulation SoC with two-way wireless telemetry for treatment of neurological disorders. VLSI Circuits (VLSIC), 2012 Symposium on, pp. 70-71.

30. Hyun Jae Baek and Hyun Seok Kim and Jeong Heo and Yong Gyu Lim and Kwang,Suk Park. (2013). Brain-computer interfaces using capacitive measurement of visual or auditory steady-state responses. Journal of Neural Engineering, 10(2), 024001 .

31. Hyung-Min Lee, Hangue Park, \& Ghovanloo, M. (2013). A power-efficient wireless system with adaptive supply control for deep brain stimulation. Solid-State Circuits, IEEE Journal of, 48(9), 2203-2216. 
32. Hyung-Min Lee, Ki-Yong Kwon, Wen Li, \& Ghovanloo, M. (2014). 24.2 A powerefficient switched-capacitor stimulating system for electrical/optical deep-brain stimulation. Solid-State Circuits Conference Digest of Technical Papers (ISSCC), 2014 IEEE International, pp. 414-415.

33. Jongwoo Lee, Hyo-Gyuem Rhew, Kipke, D. R., \& Flynn, M. P. (2010). A 64 channel programmable closed-loop neurostimulator with 8 channel neural amplifier and logarithmic ADC. Solid-State Circuits, IEEE Journal of, 45(9), 1935-1945.

34. Standard ECG signal with "PQRST" component [online] available: https://fenix.tecnico.ulisboa.pt/downloadFile/3779576309633/lab2_ps1011i.pdf

35. "Cardiovascular diseases (CVDs)", World Health Organization (WHO) [online] available at: http://www.who.int/mediacentre/factsheets/fs317/en/

36. Taillefer, Raymond, et al. "Comparative diagnostic accuracy of Tl-201 and Tc-99m sestamibi SPECT imaging (perfusion and ECG-gated SPECT) in detecting coronary artery disease in women." Journal of the American College of Cardiology 29.1 (1997): 69-77.

37. I. Stojmenovic, "Machine-to-Machine Communications With In-Network Data Aggregation, Processing, and Actuation for Large-Scale Cyber-Physical Systems," IEEE IoT-J., vol.1, no.2, pp.122,128, Apr. 2014.

38. S. Hong, D. Kim, M. Ha, S. Bae, S.J. Park, W. Jung and J. E. Kim, "SNAIL: an IPbased wireless sensor network approach to the internet of things," IEEE Wireless Commun., vol.17, no.6, pp.34,42, Dec. 2010.

39. Q. Wu, G. Ding, Y. Xu, S. Feng, Z. Du, J. Wang and K. Long, "Cognitive Internet of Things: A New Paradigm Beyond Connection," IEEE IoT-J., vol.1, no.2, pp.129,143, Apr. 2014.

40. J. Huang, Y. Meng, X. Gong, Y. Liu, and Q. Duan, "A Novel Deployment Scheme for Green Internet of Things," IEEE IoT-J., vol.1, no.2, pp.196,205, Apr. 2014.

41. J. Jin, J. Gubbi, S. Marusic, and M. Palaniswami, "An Information Framework for Creating a Smart City Through Internet of Things," IEEE IoT-J., vol.1, no.2, pp.112,121, Apr. 2014. 
42. J. Gubbi, R. Buyya, S. Marusic, and M. Palaniswami, "Internet of Things (IoT): A vision, architectural elements, and future directions", Future Gen. Comp. Sys., Vol. 29, Issue 7, pp 1645,1660, Sep. 2013.

43. C. Wang, M. Daneshmand, M. Dohler, X. Mao, R. Q. Hu and H. Wang, "Guest Editorial-Special Issue on Internet of Things (IoT): Architecture, Protocols and Services," IEEE, Sensors J., vol.13, no.10, pp.3505,3510, Oct. 2013.

44. S. D. T. Kelly, N. K. Suryadevara and S. C. Mukhopadhyay, "Towards the Implementation of IoT for Environmental Condition Monitoring in Homes," IEEE, Sensors J., vol.13, no.10, pp.3846,3853, Oct. 2013.

45. Z. Fang, L. Wei, W. Chen and Y. He, "A RFID-based kindergarten intelligence security system," in Proc. ICEBE, 2012, pp.321,326.

46. S.-J. Jung, R. Myllyla and W.-Y. Chung, "Wireless machine-to-machine healthcare solution using android mobile devices in global networks," IEEE, Sensors J., vol.13, no.5, pp.1419,1424, May 2013.

47. S. Amendola, R. Lodato, S. Manzari, C. Occhiuzzi and G. Marrocco, "RFID Technology for IoT-Based Personal Healthcare in Smart Spaces," IEEE IoT-J., vol.1, no.2, pp.144,152, Apr. 2014.

48. S. Fang, L. Xu, Y. Zhu, J. Ahati, H. Pei, J. Yan and Z. Liu, "An Integrated System for Regional Environmental Monitoring and Management Based on Internet of Things," IEEE Trans., Industrial Inf., vol.10, no.2, pp.1596,1605, May 2014.

49. M. ElHelw, J. Pansiot, D. McIlwraith, R. Ali, B. Lo and L. Atallah, "An integrated multi-sensing framework for pervasive healthcare monitoring," in Proc., Pervasive Comp. Tech. for Healthcare, 2009.

50. Jason Hill, Mike Horton, Ralph Kling, and Lakshman Krishnamurthy, "The platforms enabling wireless sensor networks,” Commun. ACM, vil.47, no.6, pp. 41,46, Jun. 2004.

51. S. Dominikus, M. Aigner and S. Kraxberger, "Passive RFID technology for the internet of things," in Proc. Int. Conf. for Internet Tech and Secured Trans, pp.8,11 Nov. 2010.

52. G. Marrocco, "Pervasive electromagnetics: sensing paradigms by passive RFID technology," IEEE, Wireless Commun., vol.17, no.6, pp.10,17, Dec. 2010.

53. J. Xiaolin, F. Quanyuan, F. Taihua and L. Quanshui, "RFID technology and its applications in Internet of Things (IoT)," in Proc. 2nd Int. Conf. on Consumer Elec., Commun. and Networks, pp.1282,1285, April 2012. 
54. A. Gluhak, S. Krco, M. Nati, D. Pfisterer, N. Mitton, and T. Razafindralambo, "A survey on facilities for experimental internet of things research," IEEE, Commun. Magaz. vol.49, no.11, pp.58,67, Nov. 2011.

55. B. Khoo, "RFID- from Tracking to the Internet of Things: A Review of developments", in Proc. IEEE/ACM Int. Conf. on Green Comp. and Commun. \& Int. Conf. on Cyber, Physical and Social Comp., pp 533-538, 2010.

56. PowerID Ltd [Online]. Available: http://www.power-id.com

57. Phase IV Engineering Ltd [Online]. Available: http://www.phaseivengr.com/Technology/SensTAG\%E2\%84\%A2WirelessRFIDSens ors.aspx

58. S. Shrestha, M. Balachandran, M. Agarwal, V. V. Phoha and K. Varahramyan, "A Chipless RFID Sensor System for Cyber Centric Monitoring Applications," IEEE Trans., Microw. Theory and Techniques, vol.57, no.5, pp.1303, 1309, May 2009.

59. D. J. Yeager, P. S. Powledge, R. Prasad, D. Wetherall, and J. R. Smith, "Wirelesslycharged UHF tags for sensor data collection," in Proc. IEEE Int. Conf. RFID, Las Vegas, NV, pp. 320,327, Apr. 2008.

60. T. Unander, J. Siden, and H. E. Nilsson, "Designing of RFID-Based Sensor Solution for Packaging Surveillance Applications," IEEE, Sensors J., vol.11, no.11, pp.3009, 3018, Nov. 2011.

61. C. Youngblut, S. H. Nash, M. S. Nash, and T. Leader, "Selected Issues in DoD's Radio Frequency Identification (RFID) Implementation" Institute of Defense Analysis, IDA paper, p-4094, Apr. 2006.

62. Y. Xiao and Y. Zhang, "Surveillance and Tracking System with Collaboration of Robots, Sensor Nodes, and RFID Tags," in Proc., 18th Int. Conf. on Comp. Commun. and Networks, pp.1-6, Aug. 2009.

63. C. Namjun, S. Seong-Jun, K. Sunyoung, K. Shiho and Y. Hoi-Jun, "A 5.1- $\mu$ W UHF RFID tag chip integrated with sensors for wireless environmental monitoring," in Proc., 31st European Solid-State Circuits Conf., pp.279,282, Sept. 2005.

64. M. Chen; S. Gonzalez, V. Leung, Qian Zhang and Ming Li, "A 2G-RFID-based ehealthcare system," IEEE, Wireless Commun., vol.17, no.1, pp.37-43, Feb. 2010. 
65. Impinj, Monja-4 RFID Tag chip [Online]. Available: http://www.impinj.com/Monza_4_RFID_Tag_Chips.aspx

66. "Temperature-Sensing RFID chip could cut shipment losses", In-Pharma Technologist.com [Online]. Available: http://www.inpharmatechnologist.com/Processing/Temperature-sensing-RFID-chip-could-cutshipment-losses.

67. "RFID and real-time temperature monitoring combine", In-Pharma Technologist.com [Online]. Available: http://www.in-pharmatechnologist.com/Drug-Delivery/RFIDand-real-time-temperature-monitoring-combine.

68. A. P. Sample, D. J. Yeager, P. S. Powledge, A. V. Mamishev, and J. R. Smith, "Design of an RFID-Based Battery-Free Programmable Sensing Platform," IEEE Trans. Instrument. and Measur., vol.57, no.11, pp.2608,2615, Nov. 2008.

69. "EPCTM radio-frequency identity protocols class-1 generation-2UHFRFID protocol for communications at $860 \mathrm{MHz}-960 \mathrm{MHz}$, version 1.2.0," Specification for RFID Air Interface, EPCglobal IncTM, Oct. 2008.

70. M. S. Khan, A. Saha and H. Deng, "Design of an Acceleration Sensor Embedded RFID (SE-RFID) Tag for Highway Guardrail Monitoring," in Proc. 1st Int. Conf. on Trends in Intell. Robo. Automa. and Manufac., Commun. in Comp. and Info. Science, Vol 330, P 296,302, Dec. 2012.

71. EPCTM Generation 1 Tag Data Standards, Version 10 1.1 Rev.1.27, Standard pecification, EPCglobal IncTM, May 2005.

72. 2N7000, Fairchild Semiconductor; datasheet [online]. Available: https://www.fairchildsemi.com/ds/2N/2N7000.pdf

73. Manganese Dioxide Lithium Coin Batteries, Panasonic, datasheet [Online]. Available:http://www.panasonic.com/industrial/includes/pdf/Panasonic_Lithium_CR 2354_CR2412.pdf

74. Arm Cortex M3, STMicroelectronics, datasheet [Online]. Available: http://www.st.com/web/en/resource/technical/document/datasheet/CD00277537.pdf

75. 3-D Tilt sensor, Analog Device, datasheet [Online]. Available: https://www.sparkfun.com/datasheets/Components/ADXL330_0.pdf 
76. MAX6605MXK+T, Low Power Analog Temp Sens., Maxim Integrated, datasheet [Online]. Available: http://datasheets.maximintegrated.com/en/ds/MAX6605.pdf.

77. Pool safety device, SenTag, datasheet [Online]. Available: http://www.sentag.com/wordpress/wp-content/uploads/2010/06/SentagWristband.pdf.

78. Bazaka, Kateryna, and M. V. Jacob. "Implantable devices: issues and challenges." Electronics 2.1, 2012, pp. 1-34.

79. Sauer, Christian, et al. "Power harvesting and telemetry in CMOS for implanted devices." Circuits and Systems I: Regular Papers, IEEE Transactions on 52.12, 2005, pp. 2605-2613.

80. Le, T.; Mayaram, K.; Fiez, T., "Efficient Far-Field Radio Frequency Energy Harvesting for Passively Powered Sensor Networks," in Solid-State Circuits, IEEE Journal of , vol.43, no.5, May 2008, pp.1287-1302.

81. Ahn, D.; Ghovanloo, M., "Optimal Design of Wireless Power Transmission Links for Millimeter-Sized Biomedical Implants," in Biomedical Circuits and Systems, IEEE Trans. on, vol., no., pp..2015.

82. Sim, Zhi Wei, et al. "Compact patch antenna design for outdoor RF energy harvesting in wireless sensor networks." Progress in Electromagnetics Research 105 (2010): 273294.

83. G. Marrocco, "Gain-Optimnized Self-Resonant Meander Line Antennas for RFID Applications," IEEE Antennas and Wireless Propagation Letters, 2, 2003, pp. 302-305.

84. Kobougias, I.C.; Tatakis, E.C., "Optimal Design of a Half-Wave Cockcroft-Walton Voltage Multiplier With Minimum Total Capacitance," in Power Electronics, IEEE Trans on , vol.25, no.9, 2010, pp.2460-2468.

85. C.O. Oluigbo, A. Salma and A.R. Rezai, "Deep Brain Stimulation for Neurological Disorders," Biomed Eng. IEEE Reviews in, vol.5, no., pp.88-99, 2012

86. S. Tisch, J.C. Rothwell, P. Limousin, M.I. Hariz and D.M. Corcos, "The Physiological Effects of Pallidal Deep Brain Stimulation in Dystonia," Neural Sys. and Rehab. Eng., IEEE Trans. on, vol.15, no.2, pp.166-172, June 2007.

87. A. Schnitzler and J. Gross, "Normal and pathological oscillatory communication in the brain," Nature Reviews Neuroscience, vol. 6, pp. 285-296, 04, 2005. 
88. M. K. Lyons, "Deep Brain Stimulation: Current and Future Clinical Applications", Mayo Clinic Proceedings, 86(7), pp.662-672, 2011.

89. T. Denison, M. Morris and F. Sun, "Building a bionic nervous system," Spectrum, IEEE, vol.52, no.2, pp.32, 39, February 2015.

90. C. C. McIntyre, A. Chaturvedi, R.R. Shamir, S.F. Lempka, "Engineering the Next Generation of Clinical Deep Brain Stimulation Technology" Brain Stimulation, Vol. 8, Issue 1, Pages 21-26, January-February 2015.

91. J. K. Zao, T. T. Gan, C. K. You, C. E. Chung, Y. T. Wang, S. J. Rodríguez Méndez, T. Mullen, C. Yu, C. Kothe, C.T. Hsiao, S. L. Chu, C. K. Shieh, and T. P. Jung, "Pervasive brain monitoring and data sharing based on multi-tier distributed computing and linked data technology," Front. Hum. Neurosci, vol. 8, June 2014.

92. J. K. Zao, C. T. Lin, L. W. Ko, H. C. She, L. R. Dung and B. Y. Chen, "Natural User Interfaces: Cyber-Physical Challenges and Pervasive Applications - A Panel Discussion," Internet of Things (iThings), 2014 IEEE International Conference on, and Green Computing and Communications (GreenCom), IEEE and Cyber, Physical and Social Computing(CPSCom), IEEE, Taipei, 2014, pp. 467-469.

93. Activa PC+S, Medtronic, [online]. Available at: http://newsroom. medtronic.com/phoenix.zhtml?c=251324\&p=irolnewsArticle\&id=18 $\underline{45602 .}$.

94. J. Lee, H.-G. Rhew, D.R. Kipke and M.P. Flynn, "A 64 Channel Programmable ClosedLoop Neurostimulator with 8 Channel Neural Amplifier and Logarithmic ADC," SolidState Circuits, IEEE Journal of, vol. 45, pp. 1935-1945, 2010.

95. K. Abdelhalim, H.M. Jafari, L.Kokarovtseva, J.L. Perez Velazquez and R. Genov, "64Channel UWB Wireless Neural Vector Analyzer SOC with a Closed-Loop Phase Synchrony-Triggered Neurostimulator," Solid-State Circuits, IEEE Journal of, vol. 48, pp. 2494-2510, 2013.

96. Y. Chen, H. W. Hao, Y. X. Hu and L. M. Li, "Wireless wrist-wearable wake/sleep identification device for closed-loop deep brain stimulation", Electronics Letters, vol. 49, pp. 452-453, 2013.

97. F. Shahrokhi, K. Abdelhalim, D. Serletis, P. L. Carlen and R. Genov, "The 128Channel Fully Differential Digital Integrated Neural Recording and Stimulation 
Interface," Biomedical Circuits and Systems, IEEE Transactions on, vol. 4, pp. 149$161,2010$.

98. Snellings, D.J. Anderson and J.W. Aldridge, "Use of multichannel recording electrodes and independent component analysis for target localization in deep brain structures," in Neural Eng., Conf. Proc. First International IEEE EMBS Conference on, pp. 305308, 2003.

99. W. Xiong and A. Kansal, "Energy efficient data intensive distributed computing," in Bulletin of the IEEE Computer Society Technical Committee on Data Engineering, 2011.

100. D. Datla, X. Chen, T. R. Newman, J. H. Reed and T. Bose, "Power Efficiency in Wireless Network Distributed Computing," Vehicular Technology Conference Fall (VTC 2009-Fall), 2009 IEEE 70th, Anchorage, AK, 2009, pp. 1-5.

101. M.S. Khan, M. S. Islam and H. Deng, "Design of a Reconfigurable RFID Sensing Tag as a Generic Sensing Platform Toward the Future Internet of Things," Internet of Things Journal, IEEE, vol. 1, pp. 300-310, 2014.

102. J.A. Thompson, D. Lanctin, N.F. Ince. and A. Abosch, "Clinical implications of local field potentials for understanding and treating movement disorders," Stereotact. Funct. Neurosurg., vol. 92, pp. 251- 263, 2014.

103. Y. Perelman and R. Ginosar, "Analog frontend for multichannel neuronal recording system with spike and LFP separation," J. Neurosci. Meth., vol. 153, pp. 21-26, 2006.

104. "Heart Disease and Stroke Statistics-At-a-Glance", American Heart Association, available [online]: public/@wcm/@sop/@smd/documents/downloadable/ucm 470704.pdf

105. Davie, A. P., et al. "Value of the electrocardiogram in identifying heart failure due to left ventricular systolic dysfunction." British Medical Journal 312.7025 (1996): 222-223.

106. Stern, Shlomo, and Dan Tzivoni. "Early detection of silent ischaemic heart disease by 24hour electrocardiographic monitoring of active subjects." British heart journal 36.5, 1974.

107. Oresko, Joseph J., et al. "A wearable smartphone-based platform for real-time cardiovascular disease detection via electrocardiogram processing." IEEE Transactions on Information Technology in Biomedicine 14.3 (2010): 734-740.

108. Young Han Nam, Zeehun Halm, Young Joon Chee and Kwang Suk Park, "Development of remote diagnosis system integrating digital telemetry for medicine," Engineering in Medicine and Biology Society, 1998. Proceedings of the 20th Annual International Conference of the IEEE, Hong Kong, 1998, pp. 1170-1173 vol.3. 
109. S. Khoor, K. Nieberl, K. Fugedi and E. Kail, "Telemedicine ECG-telemetry with Bluetooth technology," Computers in Cardiology 2001, Rotterdam, 2001, pp. 585-588.

110. EPI-MEDICS, "IST-2000-26164," 2000.

111. T. Morrison, J. Silver and B. Otis, "A single-chip encrypted wireless 12-lead ECG smart shirt for continuous health monitoring," 2014 Symposium on VLSI Circuits Digest of Technical Papers, Honolulu, HI, 2014, pp. 1-2.

112. C. P. Wang, S. Y. Lee and W. C. Lai, "An RFID tag system-on-chip with wireless ECG monitoring for intelligent healthcare systems," 2013 35th Annual International Conference of the IEEE Engineering in Medicine and Biology Society (EMBC), Osaka, 2013, pp. 5489-5492.

113. J. S. Besnoff, T. Deyle, R. R. Harrison and M. S. Reynolds, "Battery-free multichannel digital ECG biotelemetry using UHF RFID techniques," RFID (RFID), 2013 IEEE International Conference on, Penang, 2013, pp. 16-22.

114. Kligfield, Paul, et al. "Recommendations for the standardization and interpretation of the electrocardiogram: part I: the electrocardiogram and its technology a scientific statement from the American Heart Association Electrocardiography and Arrhythmias Committee, Council on Clinical Cardiology; the American College of Cardiology Foundation; and the Heart Rhythm Society endorsed by the International Society for Computerized Electrocardiology." Journal of the American College of Cardiology 49.10 (2007): 1109-1127

115. N. V. Thakor and J. G. Webster, "Ground-Free ECG Recordingwith Two Electrodes," IEEE Trans on Biomed Eng, vol. BME-27, No 12, pp. 699-704, 1980.

116. Schofield, Jamie Rae. Electrocardiogram Signal Quality Comparison Between a Dry Electrode and a Standard Wet Electrode over a Period of Extended Wear. Diss. Cleveland State University, 2012. 


\section{APPENDIX A}

\section{SOURCE CODE FOR THE SE-RFID SENSING PLATFORM}

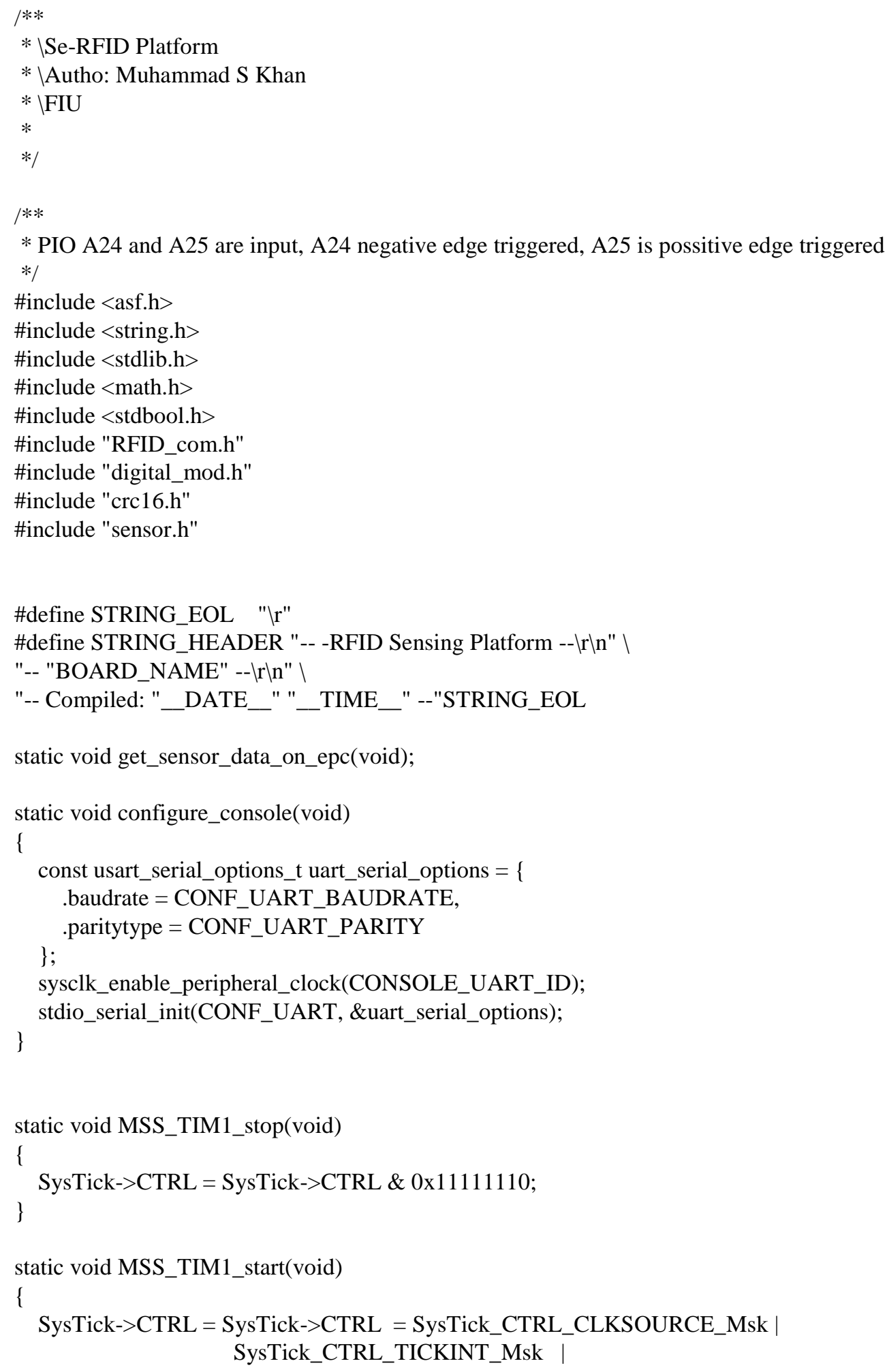




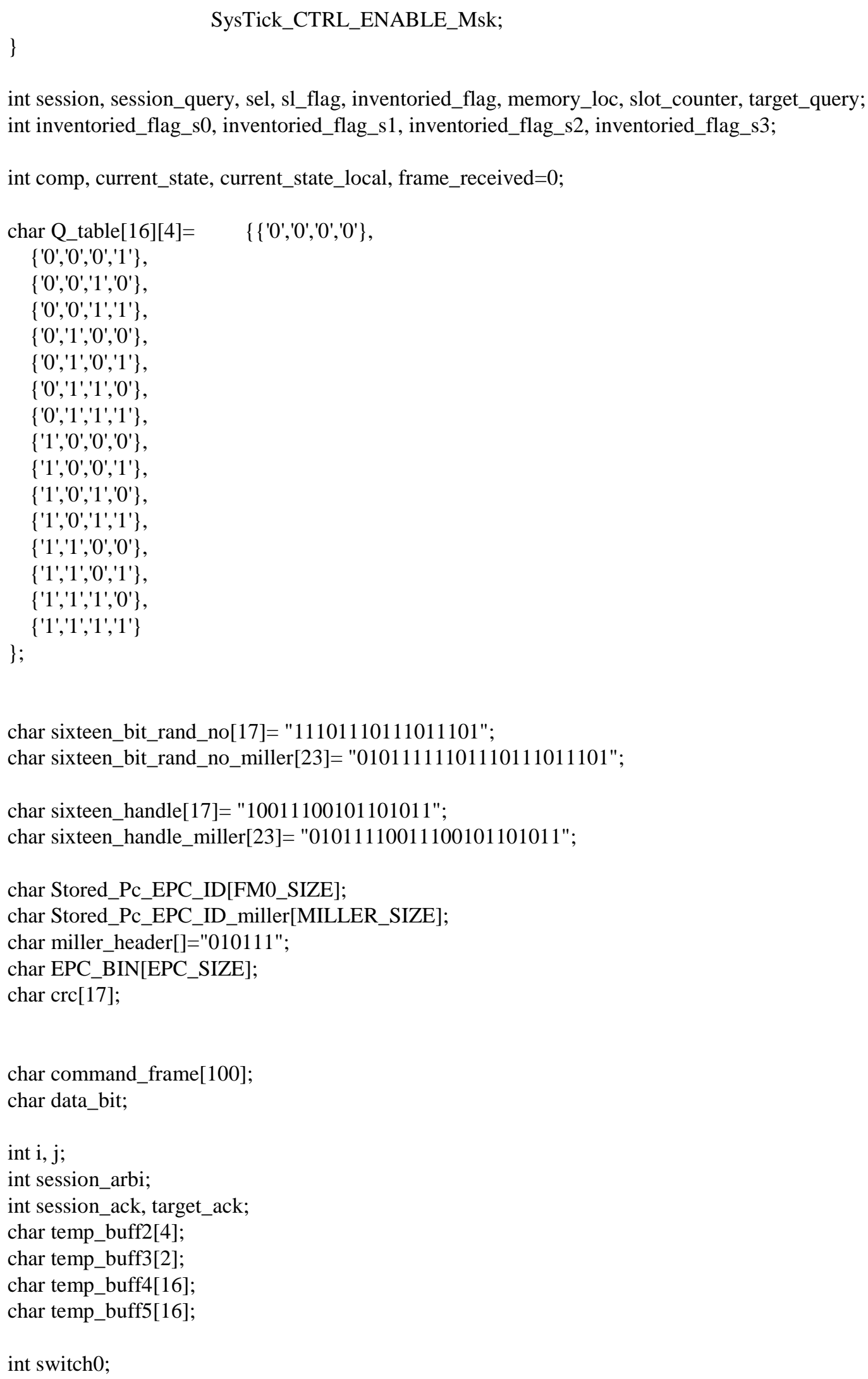




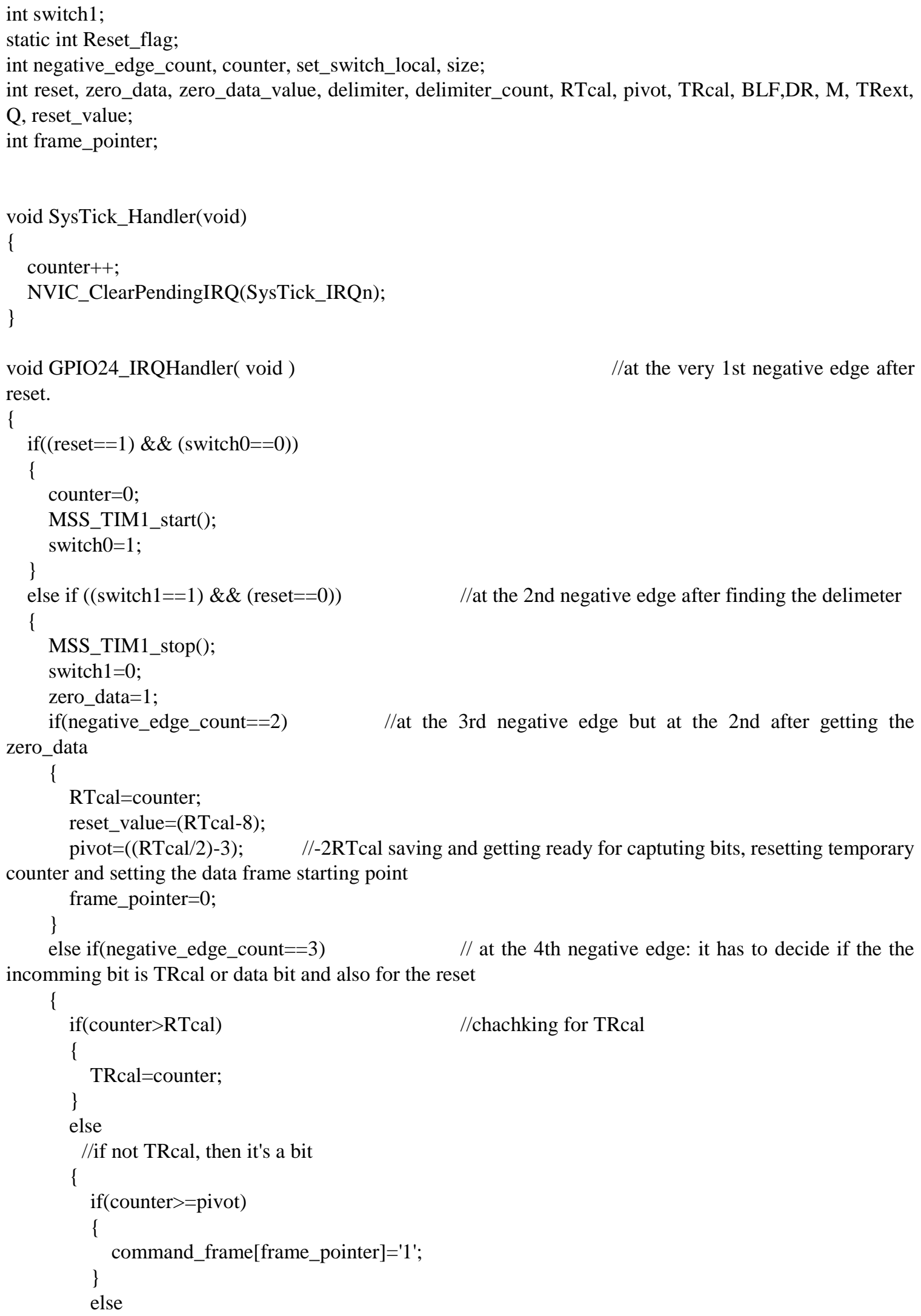




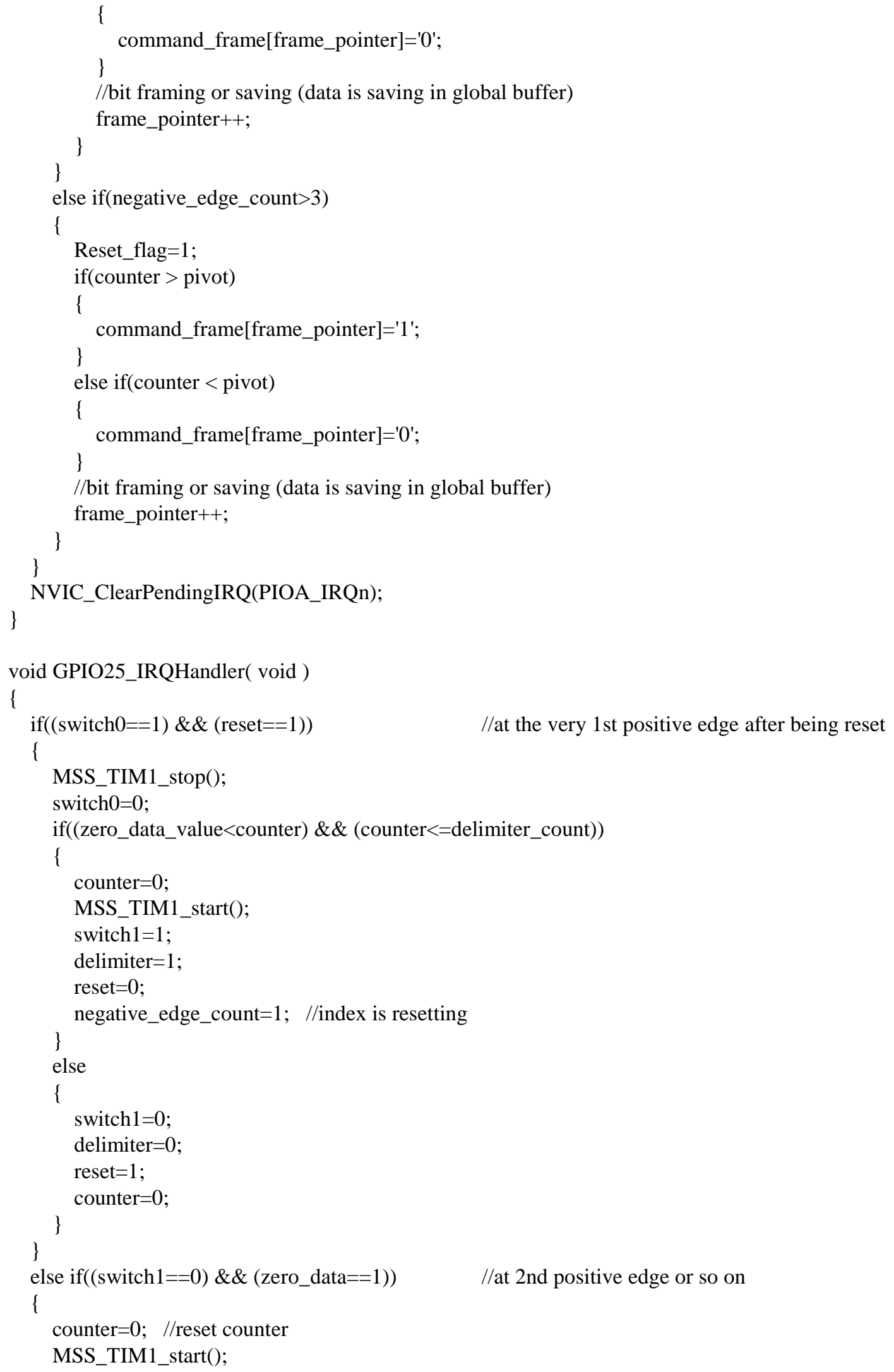




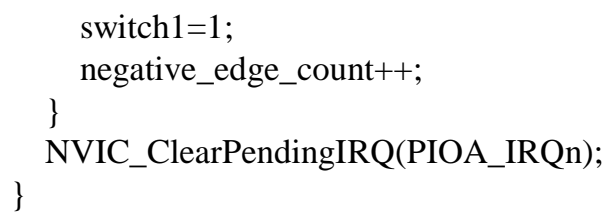


pio_pull_up(PIOA, PIO_PA25, PIO_PULLUP);

pio_configure_pin(SWT1, PIO_TYPE_PIO_INPUT);

pio_handler_set(PIOA, ID_PIOA, PIO_PA25, (PIO_IT_RISE_EDGE | PIO_DEBOUNCE | PIO_DEGLITCH), GPIO25_IRQHandler);

NVIC_EnableIRQ(PIOA_IRQn);

//pio_handler_set_priority(PIOA, PIOA_IRQn, 15); //Interrupts are 10-15, 10 is the highest priority. pio_enable_interrupt(PIOA, PIO_PA25);

//pin B0 for output

ioport_set_pin_dir(OPT, IOPORT_DIR_OUTPUT);

ioport_set_pin_level(OPT, IOPORT_PIN_LEVEL_LOW);

uint32_t cpu_freq = sysclk_get_cpu_hz();

/* Set up SysTick Timer for 1 msec interrupts. */

if (SysTick_Config(sysclk_get_cpu_hz() / 800000)) \{

while (1) \{

\}

\}

$/ *$ Initialize the sensor channel*/

init_sensor();

adc_start_software_conversion(ADC);

current_state=ready_state;

strcpy(EPC_BIN,

"00100000000000001110000000110110000100010011100110010010100001000000000000000000");

//0xE036113992840000

calculate_crc(EPC_BIN,crc);

strcpy(Stored_Pc_EPC_ID,EPC_BIN);

strcat(Stored_Pc_EPC_ID, crc);

strcat(Stored_Pc_EPC_ID, "1"); // ADD the frame end delimiter "1"

strcpy(Stored_Pc_EPC_ID_miller, miller_header);

strcat(Stored_Pc_EPC_ID_miller, EPC_BIN);

strcat(Stored_Pc_EPC_ID_miller, crc);

strcat(Stored_Pc_EPC_ID_miller, "1");

get_sensor_data_on_epc(); //needs to be turned on while the sensor option is enabled

while(1)

\{

if $(($ switch1==1) \&\& (counter>reset_value) $)$

\{

if(Reset_flag==1)

\{

MSS_TIM1_stop();

reset $=1$;

switch $0=0$;

switch $1=0$;

delimiter $=0$;

zero_data $=0$; 


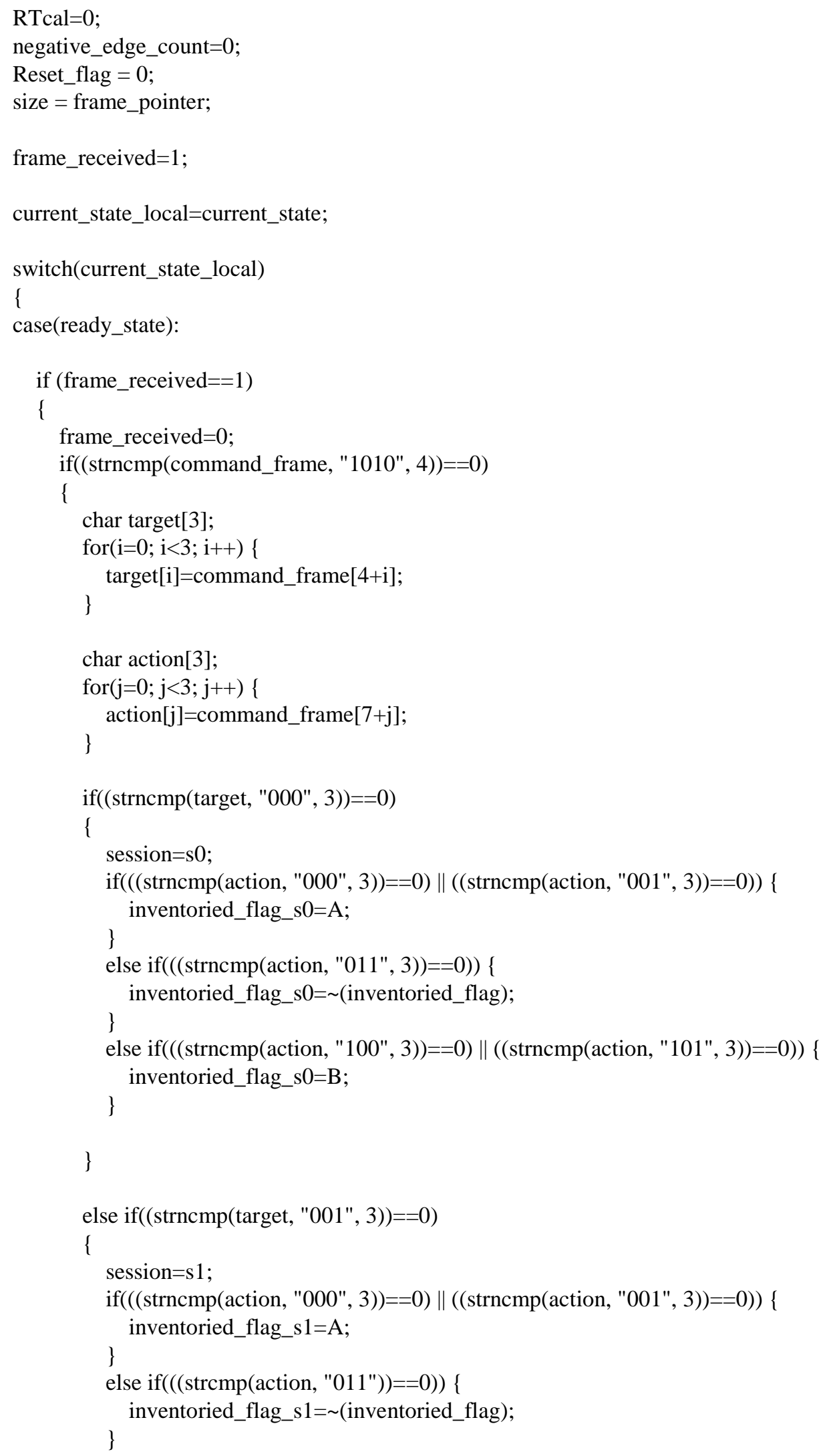




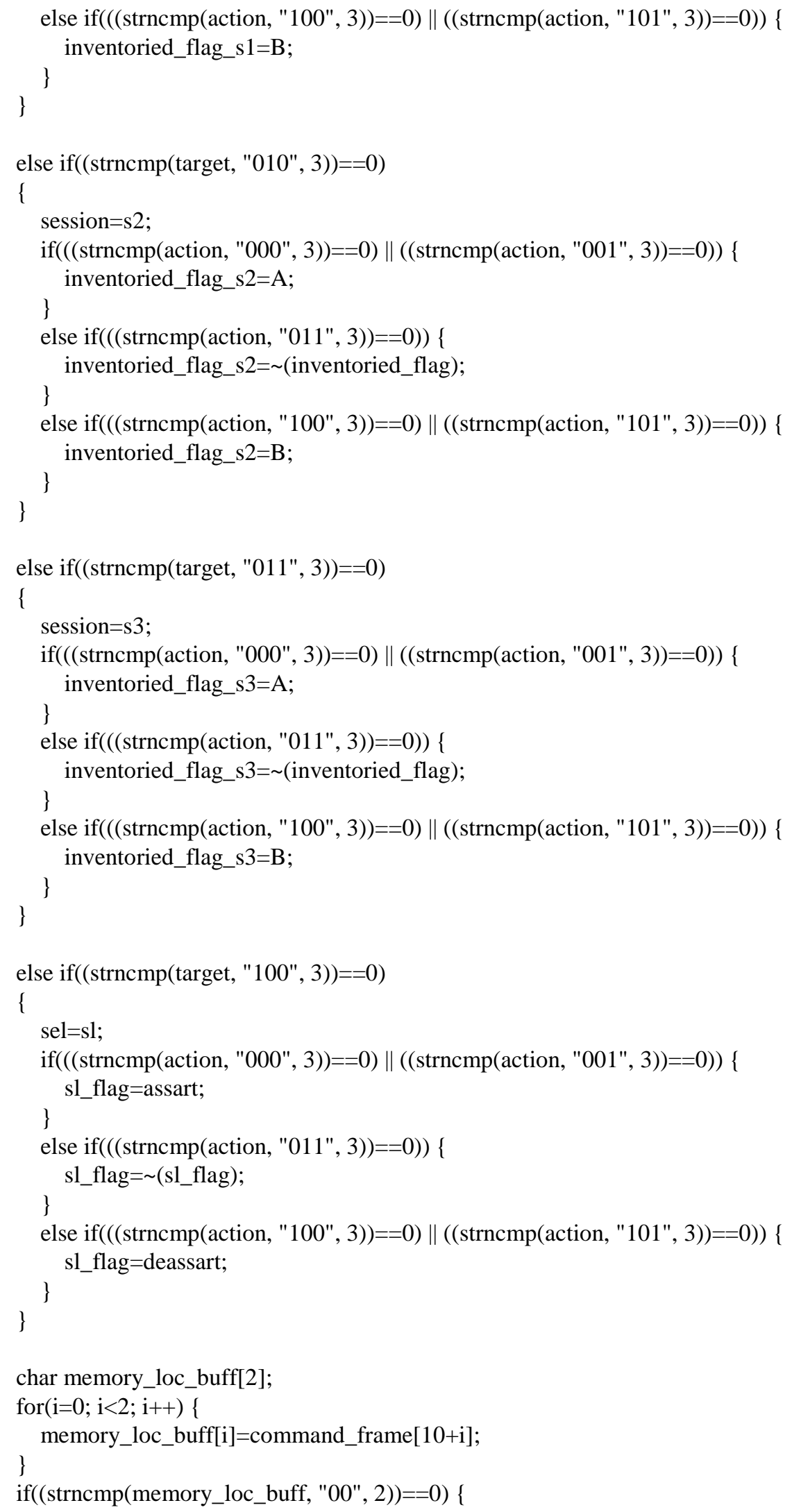




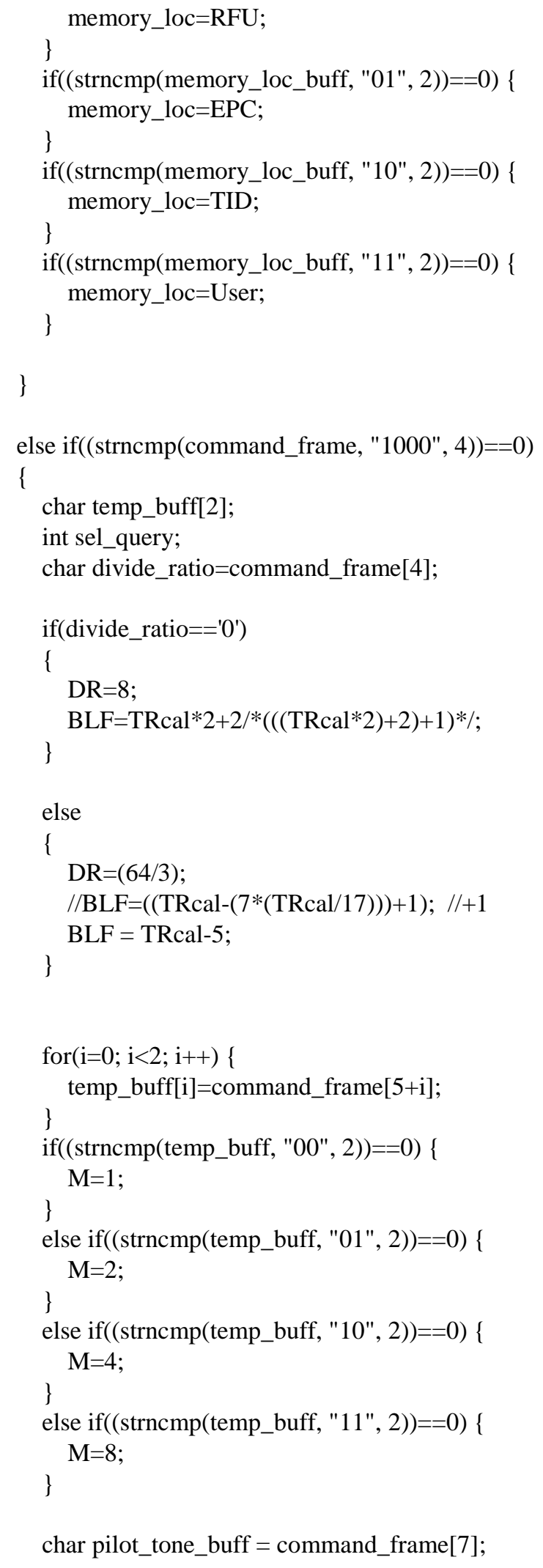




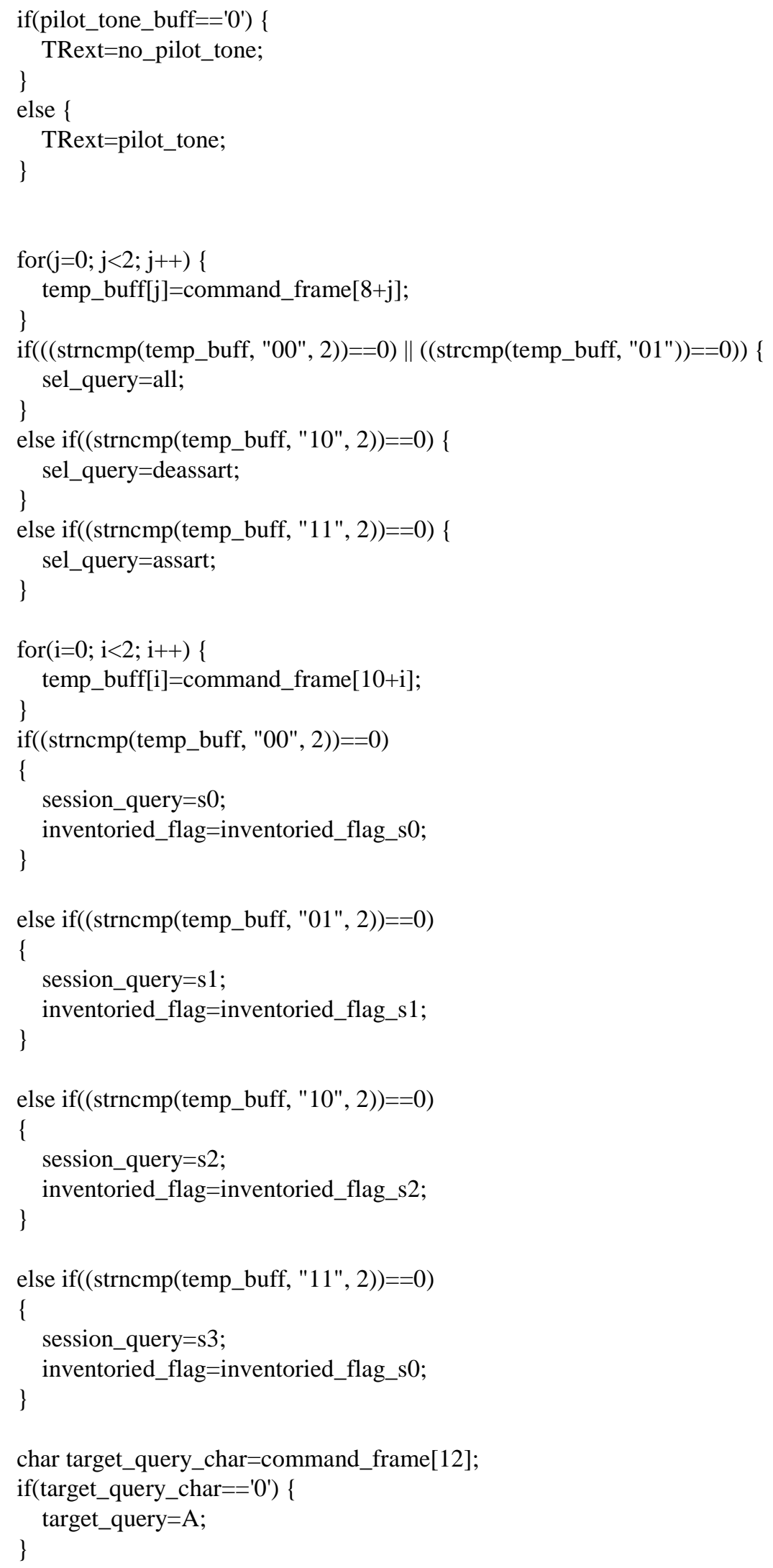




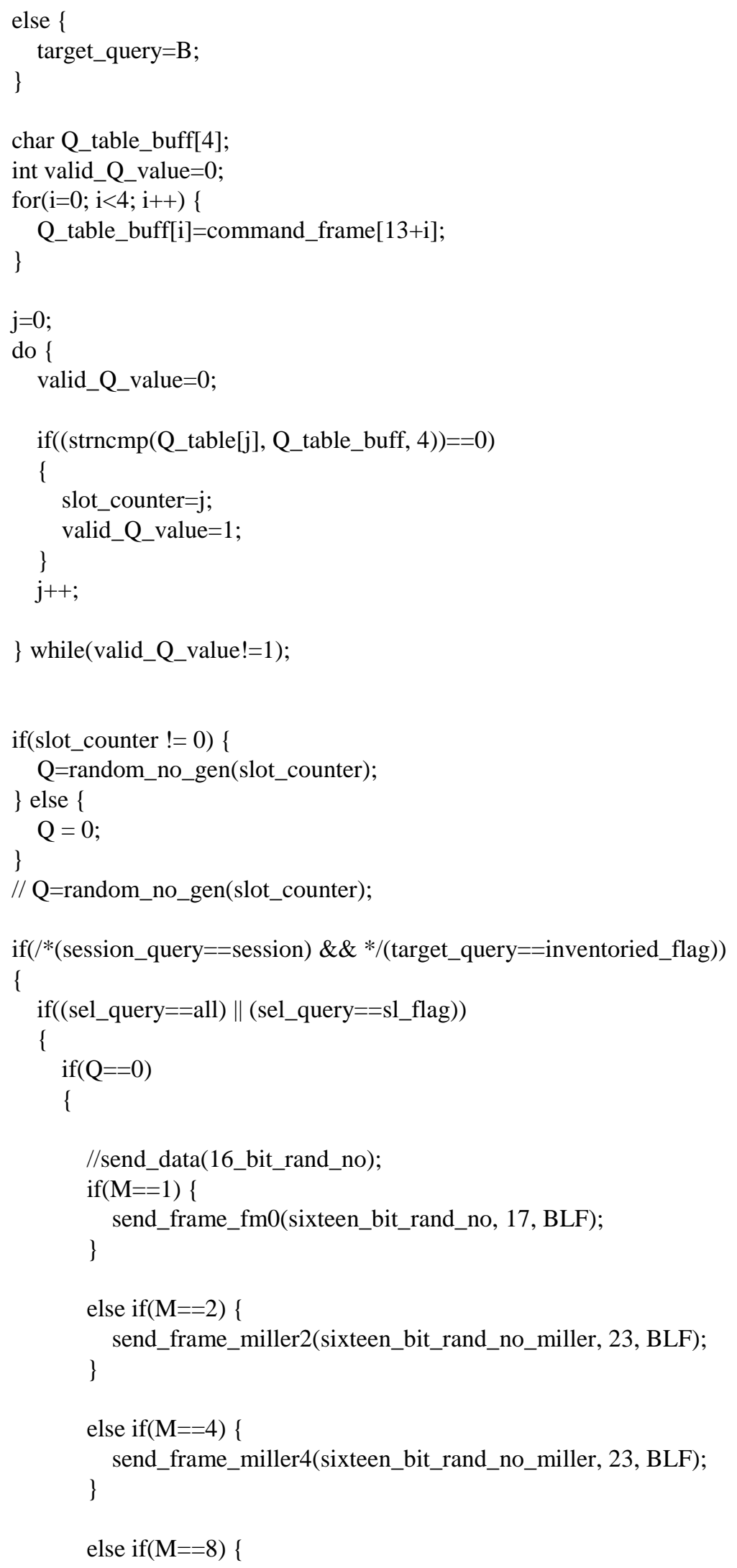




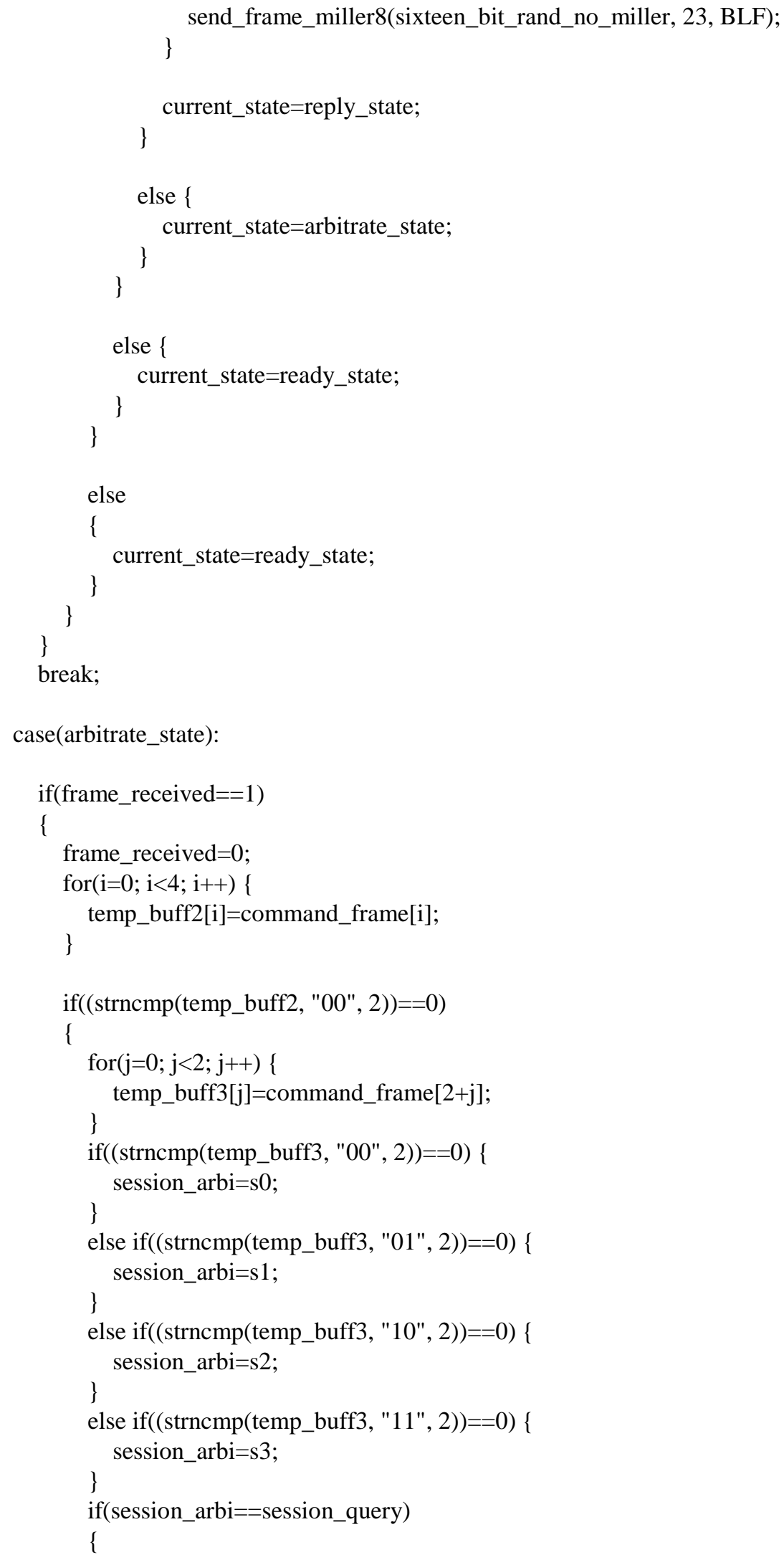




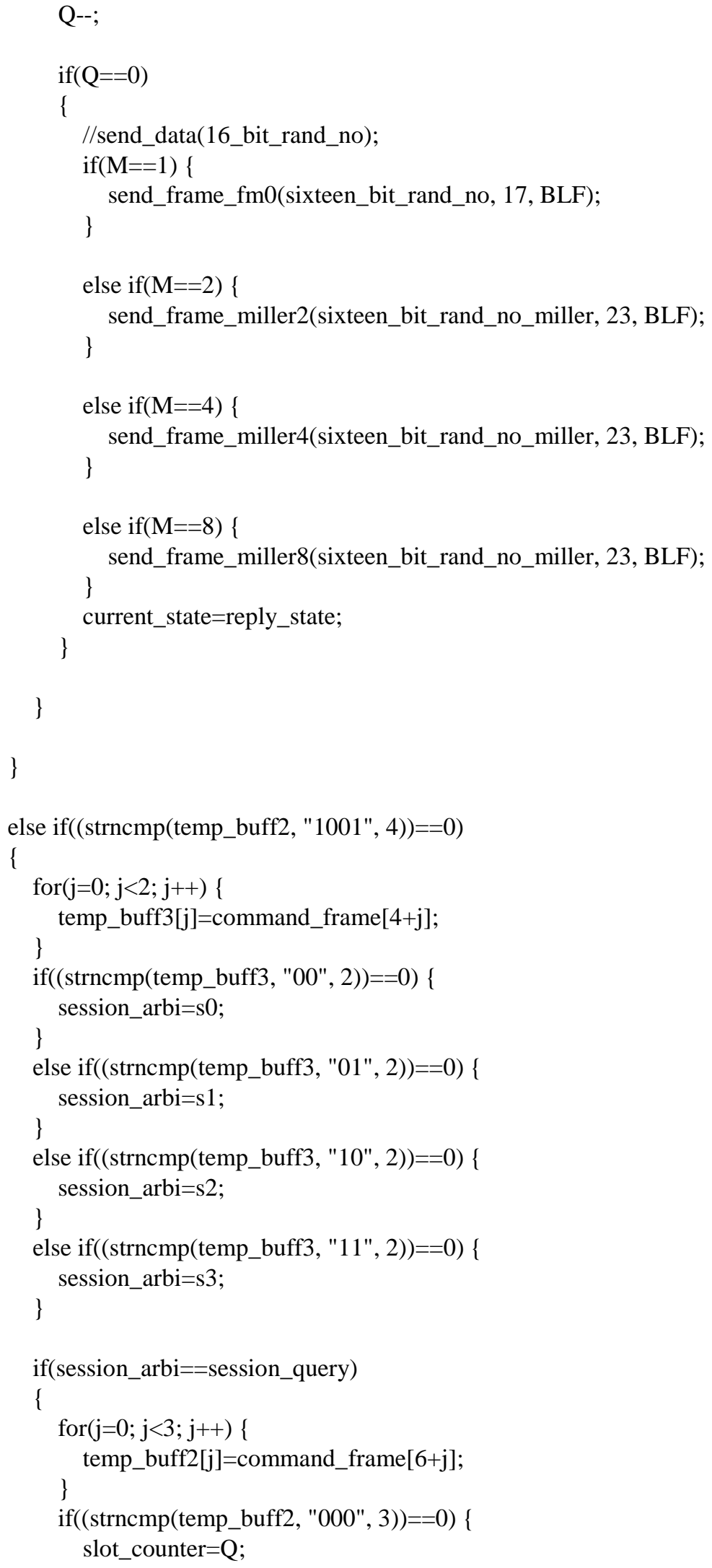




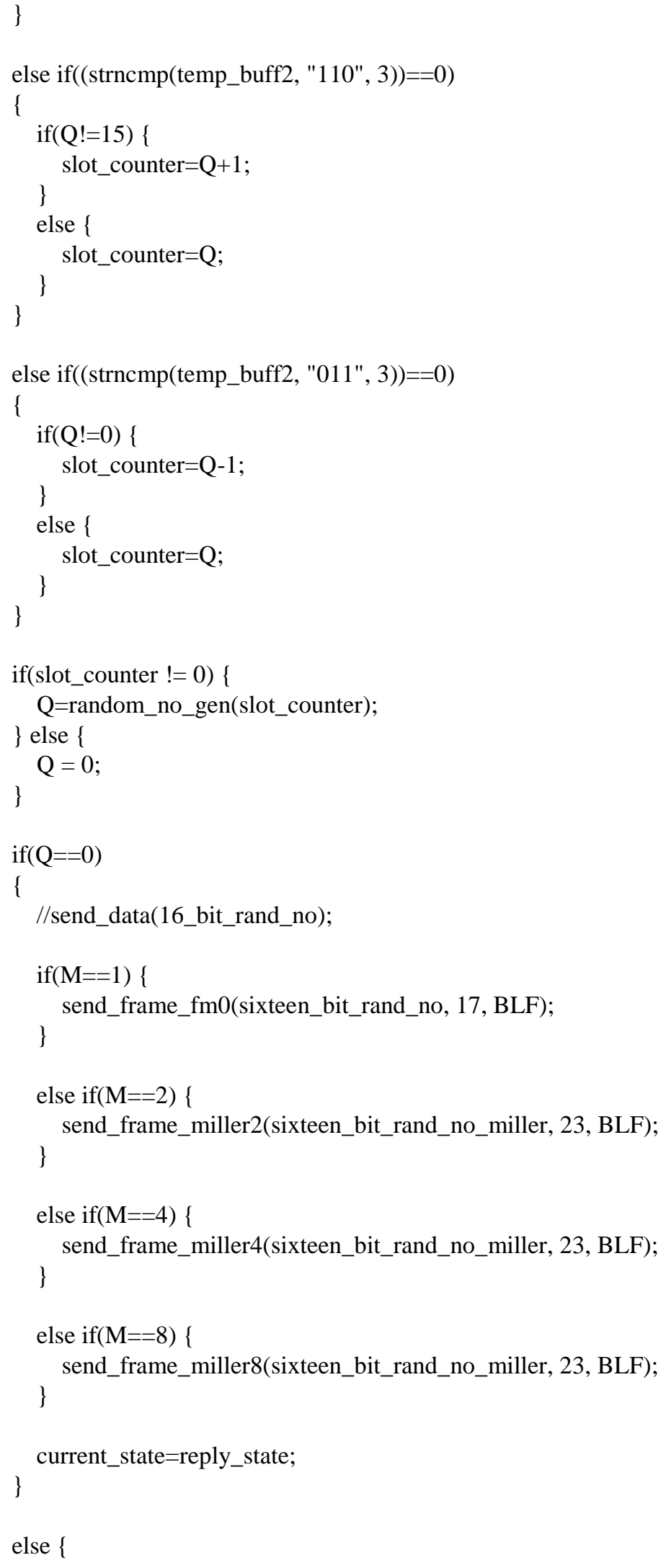




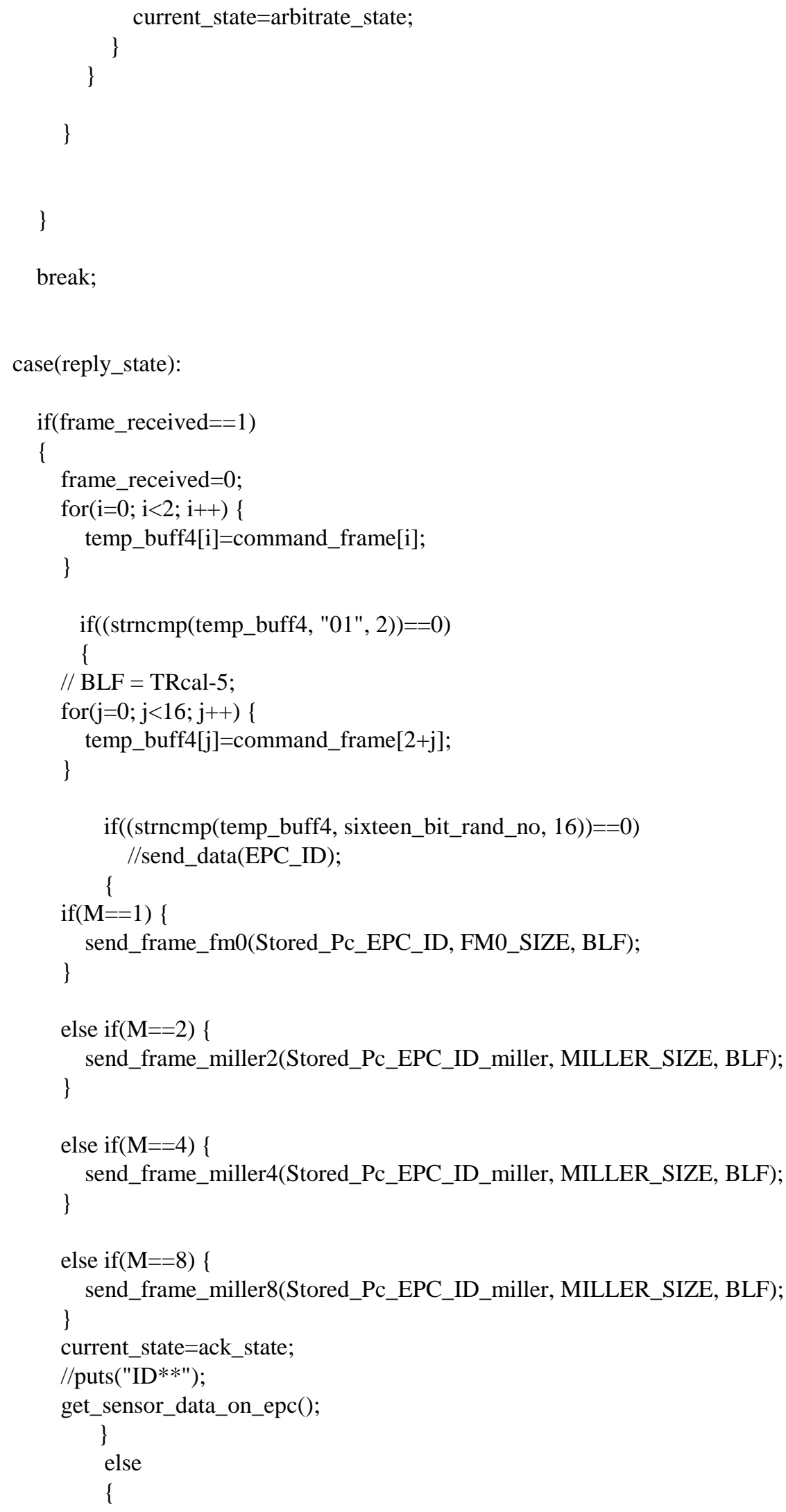


$/ /$

//

//

$/ /$

//

//

//

//

//

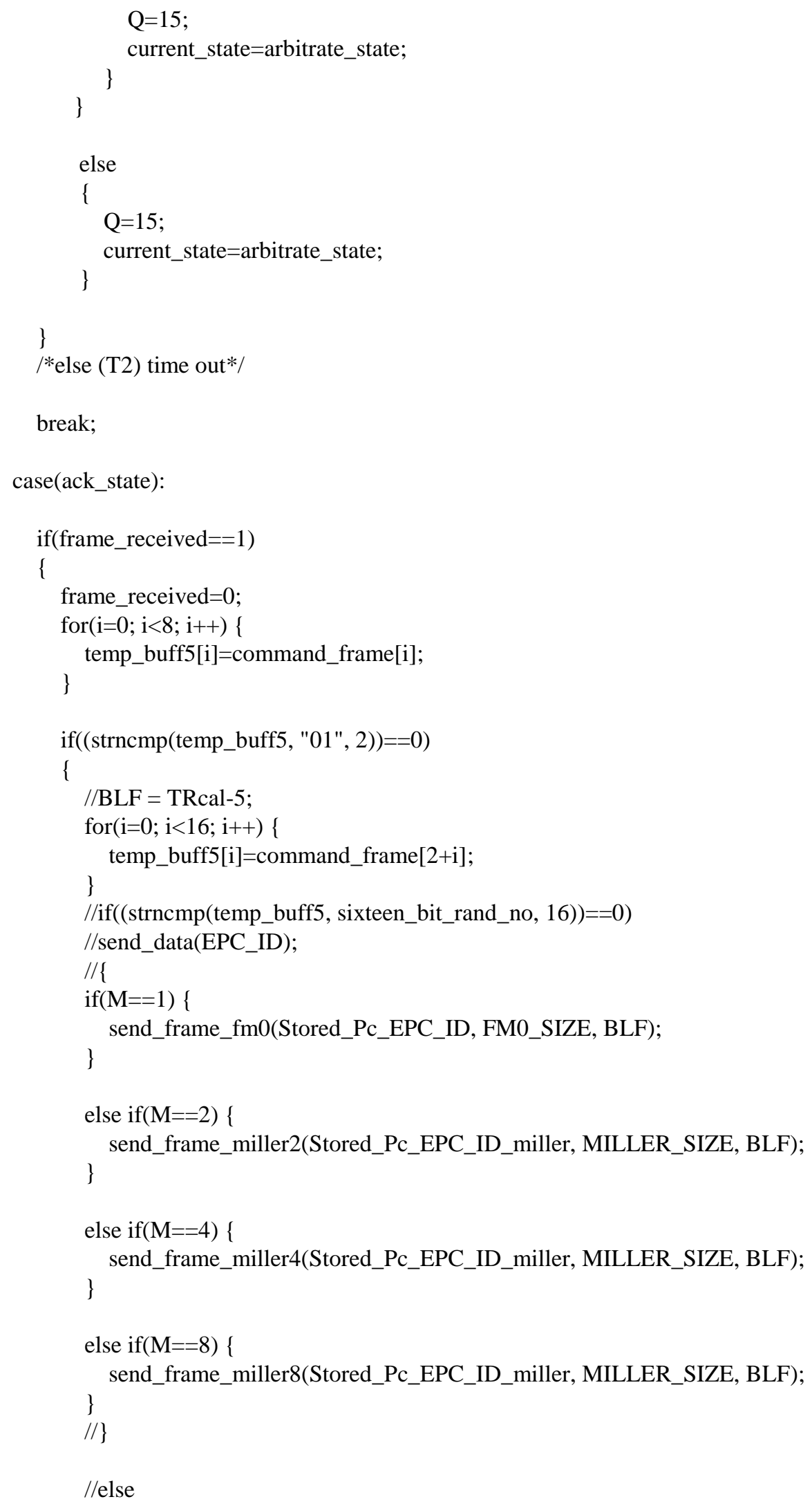




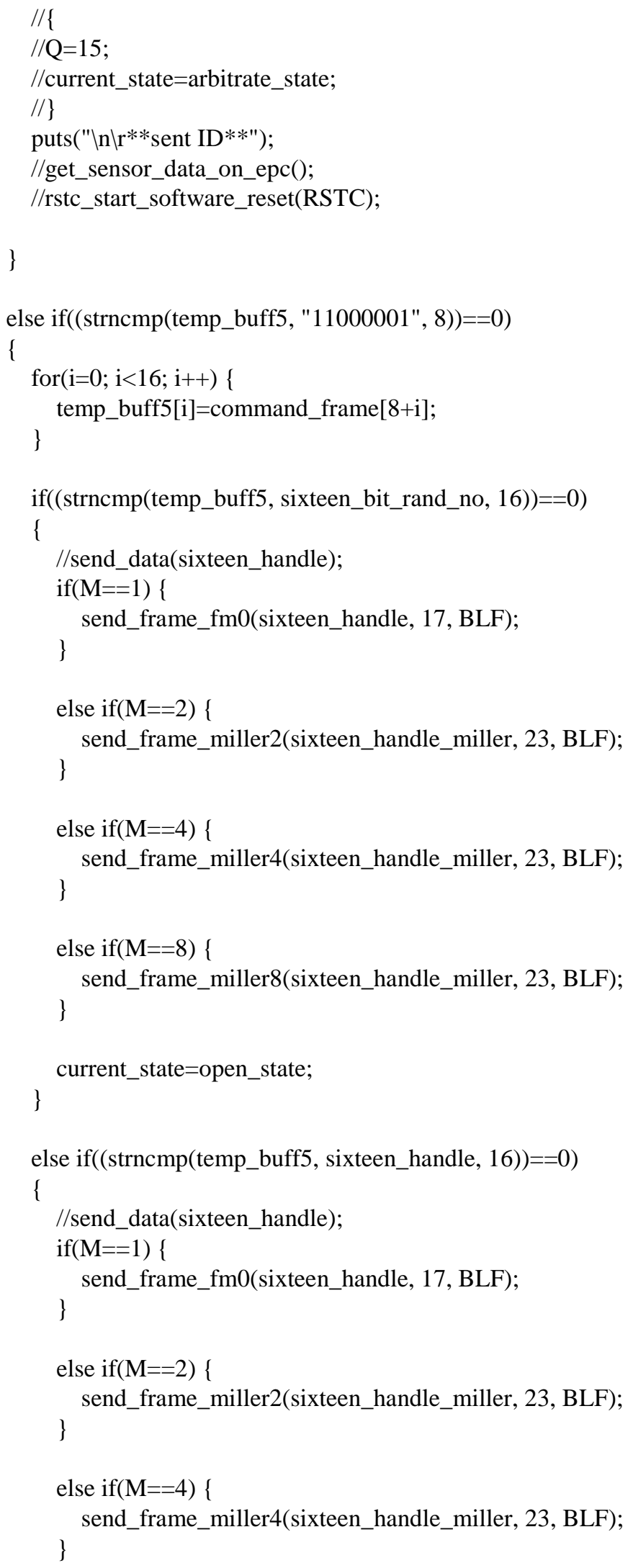




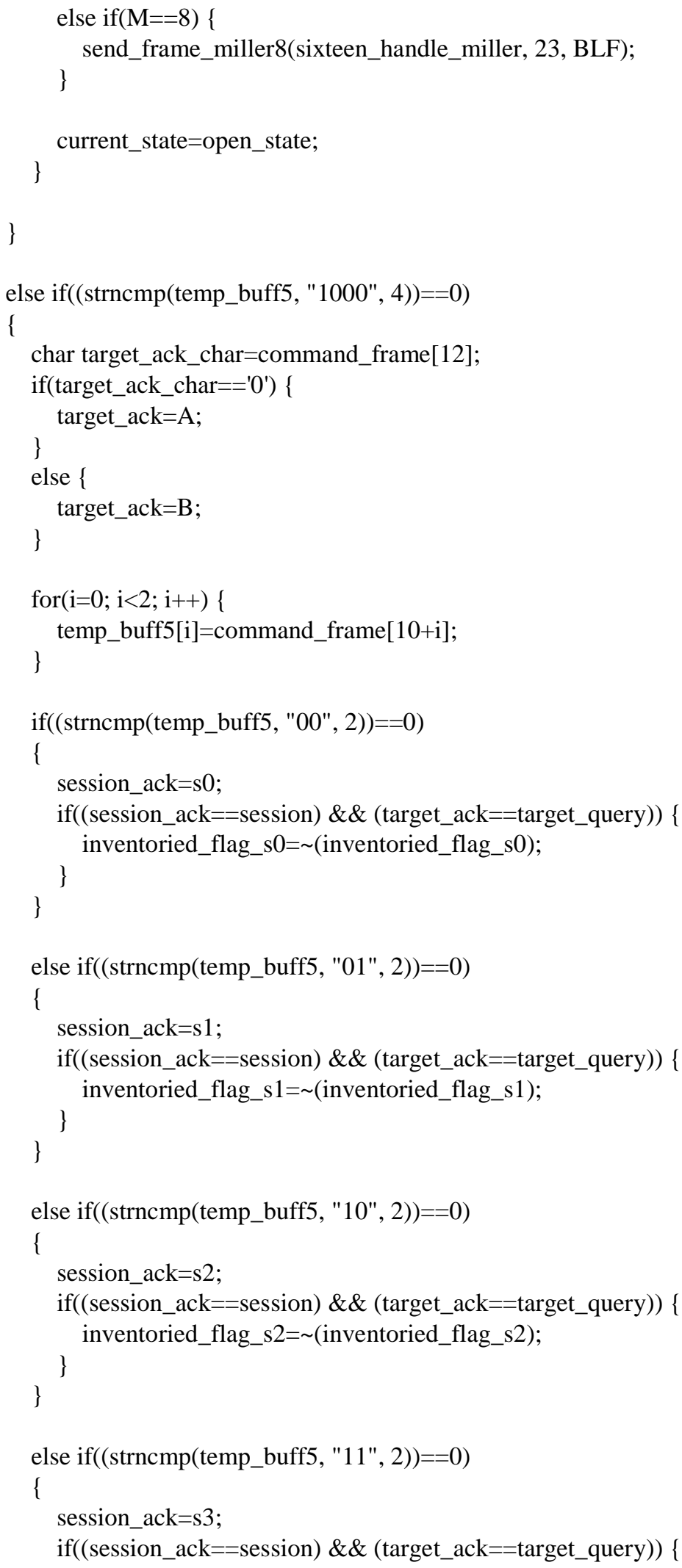




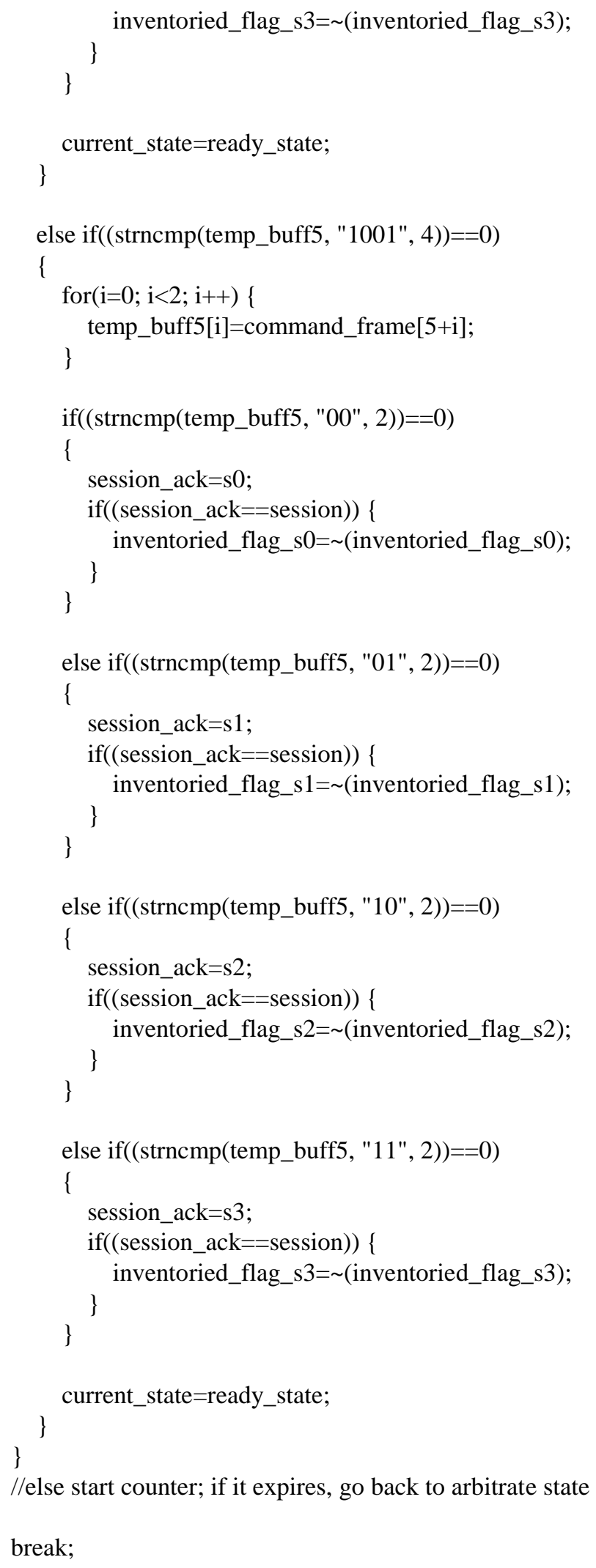




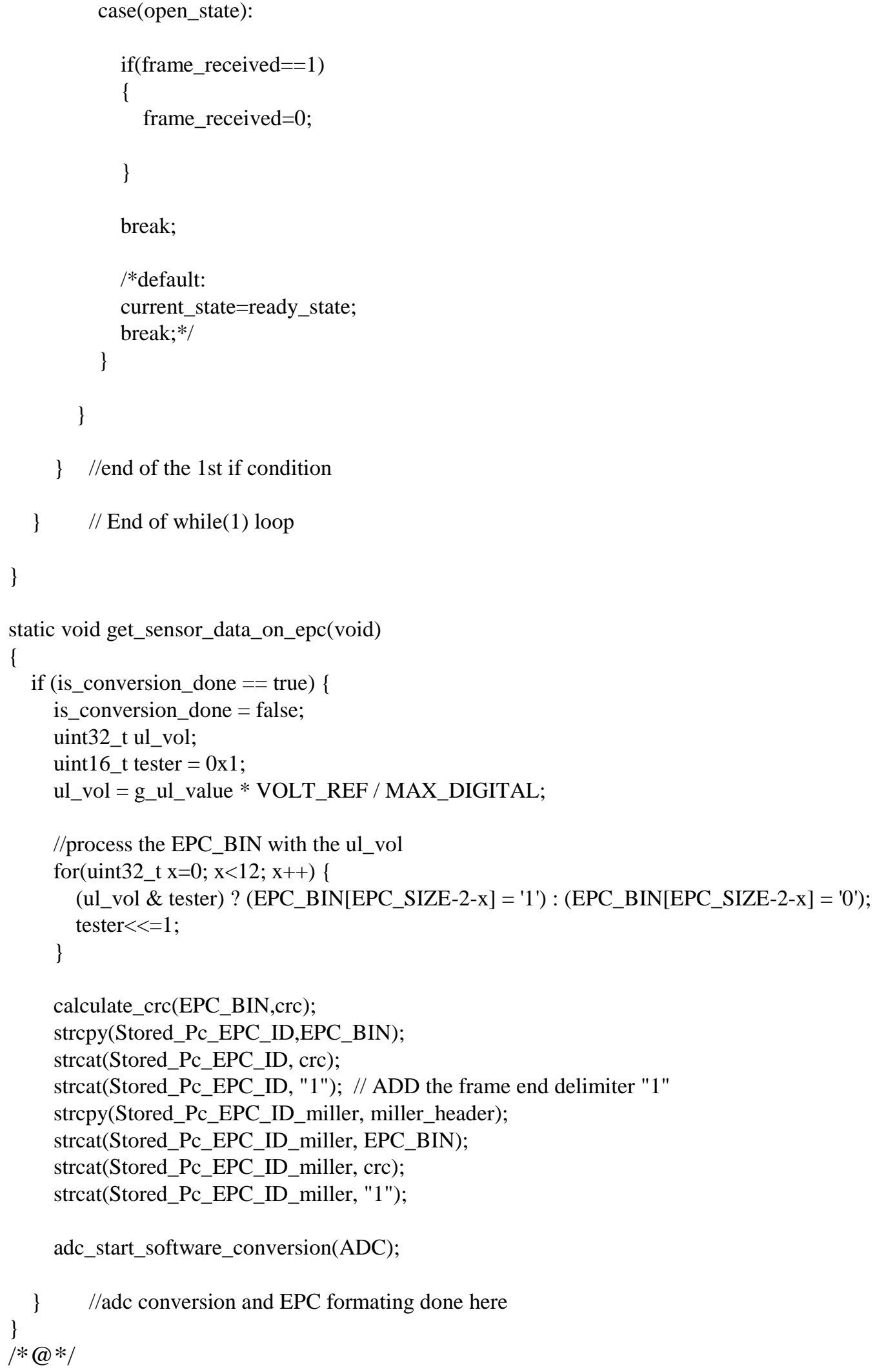


VITA

\section{MUHAMMAD S KHAN}

Miami, Florida

2004-2009

B.S., Applied Physics, Electronics and Communication Engineering

University of Dhaka

Dhaka, Bangladesh

2010-2011

M.S. Applied Physics, Electronics and Communication Engineering

University of Dhaka

Dhaka, Bangladesh

2011-2016

Doctoral Candidate, Electrical Engineering

Florida International University

Miami, Florida

\section{PUBLICATIONS AND PRESENTATION}

M.S. Khan, M. S. Islam and H. Deng, "Design of a Reconfigurable RFID Sensing Tag as a Generic Sensing Platform Toward the Future Internet of Things," Internet of Things Journal, IEEE, vol. 1, pp. 300-310, 2014.

M. S. Khan, M.S. Islam and Hai Deng, "Design and Prototyping of Smart Deep Brain Stimulator (SDBS): Towards Next-Generation Adaptive Brain-Machine-Brain Interface (BMBI)" Biomedical Circuits and Systems, IEEE Transactions on, vol., no., pp., (submitted)

M. S. Khan and Hai Deng, "Design and Implementation of A Highly Efficient UHF Energy Harvesting Antenna" in Proc. APS/URSI 2016, IEEE Int. Symp. On, Puerto Rico, Jun. 2016.

M. S. Khan, A. Saha, and Hai Deng, "Design of an acceleration sensor embedded RFID (SE-RFID) tag for highway guardrail monitoring" in Proc. 1st Int. Conf. Trends Intell. Robot. Autom. Manuf. Commun. Comput. Inf. Sci., Dec. 2012, vol. 330, pp. 296-302.

3Mohammad S Islam, Khondaker A. Mamun, M. S. Khan and Hai Deng, "Performance Assessment of Artificial Neural Network Classifier for Predicting Movement and Laterality of Deep Brain Local Field Potential," 3rd workshop on Machine learning and interpretation in neuroimaging (MLINI 2013) in Neural information processing systems (NIPS 2013), Nevada, USA, Dec 9-10, 2013. 
Mohammad S Islam, Khondaker A Mamun, M. S. Khan and Hai Deng: “A Probabilistic Neural Network Approach for Prediction of Movement and its Laterality from Deep Brain Local Field Potential (LFP)" Modern Trends and Techniques in Computer Science Pages: 129-141.

Mohammad S Islam, K A Mamun, M. S. Khan, Shouyan Wang, Hai Deng “Decoding Movements from Human Deep Brain Local Field Potentials (LFPs) Using Radial Basis Function Neural Network (RBFNN)" 27th IEEE International symposium on Computer-Based Medical Systems(CBMS 2014) May27-29, 2014, New York, USA. 